\title{
PROPOSAL: P-1101 \\ Matter-wave Atomic Gradiometer Interferometric Sensor (MAGIS-100)
}

\author{
Phil Adamson $^{1}$, Swapan Chattopadhyay ${ }^{1,2}$, Jonathon Coleman ${ }^{5}$, Peter Graham ${ }^{3}$, Steve \\ Geer $^{1}$, Roni Harnik ${ }^{1}$, Steve Hahn ${ }^{1}$, Jason Hogan ${ }^{\dagger 3}$, Mark Kasevich ${ }^{3}$, Tim Kovachy ${ }^{6}$, \\ Jeremiah Mitchell ${ }^{2}$, Rob Plunkett ${ }^{1}$, Surjeet Rajendran ${ }^{4}$, Linda Valerio ${ }^{1}$ and Arvydas \\ Vasonis ${ }^{1}$ \\ ${ }^{1}$ Fermi National Accelerator Laboratory; Batavia, Illinois 60510, USA \\ ${ }^{2}$ Northern Illinois University; DeKalb, Illinois 60115, USA \\ ${ }^{3}$ Stanford University; Stanford, California 94305, USA \\ ${ }^{4}$ University of California at Berkeley; Berkeley, CA 94720, USA \\ ${ }^{5}$ University of Liverpool; Merseyside, L69 7ZE, UK \\ ${ }^{6}$ Northwestern University; Evanston, Illinois, USA
}

December 17, 2018

\begin{abstract}
We propose to build and install at Fermilab a next generation instrument that uses light pulse atom interferometry to search for physics beyond the Standard Model. In particular, we propose to search for dark matter and new forces, and to test some aspects of quantum mechanics in a new regime. The setup will exploit the existing $\sim 100 \mathrm{~m}$ vertical NuMI access shaft, and will be an upgrade of the existing $10 \mathrm{~m}$-scale experiment at Stanford. Note that for many searches, the sensitivity of the experiment is proportional to baseline. To fully exploit the opportunity, we would use the latest advances in atomic clock technologies. The experiment would also provide an R\&D platform for, and a critical step towards, the next-but-one generation experiment, which might possibly be located in a shaft at SURF in South Dakota, and which would be sufficiently sensitive to detect, in an unexplored frequency range, gravitational waves from known sources.
\end{abstract}

${ }^{0} \dagger$ contact person

This manuscript has been authored by Fermi Research Alliance, LLC under Contract No. DE-AC02-07CH11359 with the U.S. Department of Energy, Office of Science, Office of High Energy Physics. 


\section{Contents}

1 Introduction $\quad 4$

2 Physics Motivation $\quad \mathbf{5}$

2.1 Dark Matter . . . . . . . . . . . . . . . . . . . . 5

2.2 New Forces . . . . . . . . . . . . . . . . . . . . . . . 7

2.3 Quantum Science . . . . . . . . . . . . . . . . . . . . . . 8

2.4 Gravitational Waves . . . . . . . . . . . . . . . . . . . . . . 8

3 Experimental Overview $\quad 11$

3.1 A light pulse atom interferometer . . . . . . . . . . . . . . 11

3.2 A Gradiometer . . . . . . . . . . . . . . . . . . . . . . . 12

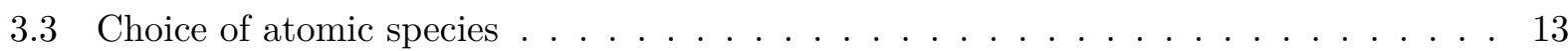

3.4 Using advanced atom optics . . . . . . . . . . . . . . . . . . . 15

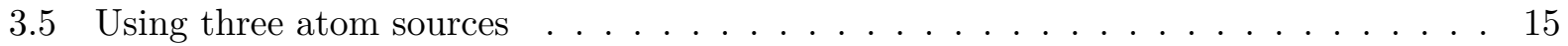

4 Present generation (10 m-scale) experiment \& illustrative results 16 4.1 Results from the Stanford Experiment, a 10 m Fountain . . . . . . . . . . . . . . 18

5 Technological advances required for MAGIS-100 20

6 Requirements for a 100 m-scale experiment 21

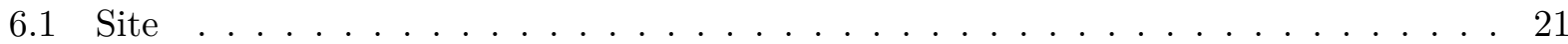

6.2 Vacuum and Vacuum pipe . . . . . . . . . . . . . . . . . . 21

6.3 Magnetic shielding and magnetic field control . . . . . . . . . . . . . . . . 22

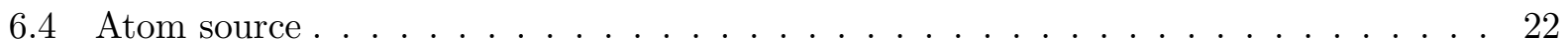

6.5 Transfer and Launch . . . . . . . . . . . . . . . . . . . . . 23

6.6 Atom optics laser system . . . . . . . . . . . . . . . . . . 24

6.7 Laser wavefront aberrations . . . . . . . . . . . . . . . . 25

6.8 Tip-tilt mirrors and rotation compensation $\ldots \ldots \ldots \ldots \ldots$

6.9 Controls and monitoring . . . . . . . . . . . . . . . . 27

6.10 Cameras and Data Acquisition . . . . . . . . . . . . . . . 27

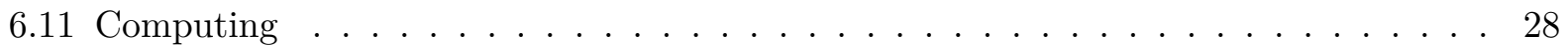

7 MAGIS-100 at Fermilab 28

7.1 Layout . . . . . . . . . . . . . . . . . . . . . 28

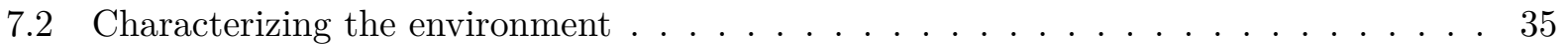

7.3 Other site-specific considerations . . . . . . . . . . . . . . 35

7.4 Error Modeling . . . . . . . . . . . . . . . . . . . . . 37

8 Development plan $\quad 37$

8.1 Large momentum transfer atom optics . . . . . . . . . . . . . . . . . . . . . . . . . . . . 39

8.2 High flux atom sources . . . . . . . . . . . . . . . . . . . . 39

8.3 Spin-squeezed sources . . . . . . . . . . . . . . . . . . . 39 
9 Responsibilities, resource requirements, and timeline $\quad 40$

9.1 Responsibilities . . . . . . . . . . . . . . . . . . . . 40

9.2 Procurements . . . . . . . . . . . . . . . . . . . . 42

9.3 Utilization of laboratory infrastructure . . . . . . . . . . . . . . . . . 42

9.4 Cost Estimate . . . . . . . . . . . . . . . . . . . . . . 43

9.5 Technically Driven Timeline . . . . . . . . . . . . . . . . . . 44

9.6 Towards a Risk Register . . . . . . . . . . . . . . . . . . . . . 47

10 Interactions with Funding Agencies $\quad 47$

A Appendix: Fermilab contributions $\mathbf{5 5}$

A.1 Proposed Fermilab Contributions . . . . . . . . . . . . . . . . 55

A.2 Fermilab Facilities . . . . . . . . . . . . . . . . . . . 55

B Appendix: Stanford contributions $\quad 55$

B.1 Proposed Stanford Contributions . . . . . . . . . . . . . . . . 56

C Appendix: Northern Illinois University contributions 56

C.1 Proposed NIU Contributions . . . . . . . . . . . . . . . . . 56

D Appendix: Northwestern contributions $\quad \mathbf{5 7}$

D.1 Proposed Northwestern Contributions . . . . . . . . . . . . . . . . 57

E Appendix: Berkeley contributions $\quad \mathbf{5 7}$

E.1 Proposed Berkeley Contributions . . . . . . . . . . . . . . . . . 57

F Appendix: University of Liverpool contributions $\quad \mathbf{5 7}$ F.1 Proposed Liverpool Contributions _. . . . . . . . . . . . . . . . 58

G Appendix: MAGIS-1000, a long-term vision $\quad 59$

$\begin{array}{ll}\text { H Appendix: Systematics and Error Modeling } & 61\end{array}$

$\begin{array}{lll}\text { I Appendix: Gravity Gradient Noise } & 68\end{array}$

J Appendix: Laser Wavefront Aberration $\quad 69$

J.1 Calculation . . . . . . . . . . . . . . . . . . . . . . . . . . 69

J.2 Leading Order Terms . . . . . . . . . . . . . . . . . . . . . . . 71

J.3 Wavefront Imaging . . . . . . . . . . . . . . . . . . . 71 


\section{Introduction}

Light-pulse atom interferometers have been shown to be capable of searching for new physics with exquisite precision. Members of the MAGIS-100 Collaboration have built and operated a lightpulse atom interferometer in which a cloud of cold atoms rises and falls within a $10 \mathrm{~m}$ vertical pipe [1]. As the atom cloud falls, a series of laser pulses interacts with the cloud to create an atom interferometer. The interferometer is sensitive to new physics. The way this works is described in Section 3. For many searches for new physics, the sensitivity of the interferometer is proportional to its baseline, and hence the fall distance. We propose to build the next-generation instrument by increasing the baseline by an order of magnitude. This requires a $\sim 100 \mathrm{~m}$ long vertical shaft located in a laboratory environment capable of providing the infrastructure required for the experiment. The NuMI vertical access shaft at Fermilab is suitable for this next-generation experiment.

We propose to build and operate the MAGIS- $100^{1}$ experiment in the NuMI shaft at Fermilab. With three atom sources the experiment would be operated in a number of configurations, each optimized for a particular physics measurement. In its simplest configuration the setup would use two of the atom sources and consist of two identical interferometers, one located near the top of the NuMI shaft and the other near the bottom. The interferometers would therefore be separated by $\sim 100 \mathrm{~m}$ but would be connected via a common set of laser pulses. This configuration can be thought of as a quantum sensor network with two nodes.

Several community reports [2-4] have recognized that quantum sensor networks have a broad scientific potential. In particular, [2] noted their potential use in searching for new fundamental forces, dark matter and other dark sector ingredients. These networks also offer the possibility of testing quantum mechanics over record breaking macroscopic distances and timescales, and of searching for gravitational waves in an unexplored frequency range.

The goals of the proposed experiment are to:

- Explore new regions of dark-sector parameter-space, and hence extend the search for dark matter.

- Search for new forces.

- Advance the frontier of quantum sensor technologies and the quantum science that can be pursued with those technologies, including quantum tests on record-setting macroscopic scales.

- Provide a critical step towards a next-but-one generation instrument, which would enable another step in sensitivity to new physics, and also be sensitive to gravitational radiation in an unexplored frequency band. A candidate location for a $1 \mathrm{~km}$ scale experiment might be a vertical shaft at the SURF laboratory in South Dakota.

Design, construction, and integration of the main components of the detector is expected to take 2-3 years. The science program will then take an additional 3 years.

The physics motivation for MAGIS-100 is described in more detail in Section 2. Section 3 describes the generic ingredients of a light pulse atom interferometer, and how this instrument is used to search for new physics. Section 4 describes the present generation experiment and some results that illustrate its capabilities. Section 5 summarizes the technical advances required for a $100 \mathrm{~m}$ scale experiment. In Section 6 the requirements for a $100 \mathrm{~m}$ scale experiment are described. Section 7 describes the proposed MAGIS-100 setup at Fermilab. Section 8 discusses the R\&D plan that will facilitate the ultimate MAGIS-100 sensitivity. Section 9 outlines the main institutional

\footnotetext{
${ }^{1}$ Matter-wave Atomic Gradiometer Interferometric Sensor with a $100 \mathrm{~m}$ baseline
} 
responsibilities, the required resources, and the hoped-for time-line for building the experiment. Finally, Section 10 summarizes interactions with the funding agencies.

\section{Physics Motivation}

In the following we describe the motivation for the proposed experiment.

\subsection{Dark Matter}

Cosmological and astronomical measurements have conclusively established that the energy budget of the Universe is dominated by dark energy and dark matter. Discovering the properties of these unknown constituents of the Universe is one of the priorities for the DOE OHEP supported program. The P5 report [5] recommended investment in small projects to explore this sector. The key role of quantum sensors in this area was highlighted in the community reports [2-4].

Dark matter can lead to time dependent signals in high precision quantum sensor networks, enabling a unique probe of its existence. In particular, these time dependent signals can be caused by ultra-light dark matter candidates. Observational bounds permit the mass of dark matter to be as low as $10^{-22} \mathrm{eV}$, whereas current experiments have focused on dark matter in a narrow range of masses (e.g. around $100 \mathrm{GeV}$ for WIMPs). Given the null results from the present generation of dark matter experiments, it is important to broaden the search to cover a wider range of dark matter candidates. Well motivated theories indicate that the mass range from $10^{-22} \mathrm{eV}$ to $10^{-3} \mathrm{eV}$ is particularly interesting. Potential dark matter candidates within this range include the QCD axion, axion-like-particles, and the relaxion. Dark matter in this mass range has a large number density and can be described as a classical field that oscillates at a frequency determined by the mass of the dark matter particle. This results in time dependent effects that can be searched for using a quantum network. These effects arise because as the classical dark matter field oscillates, the properties of the sensor (such as the quantum energy level and spin) also change, leading to time dependent signals. The fact that the dark matter signal oscillates at a frequency set by fundamental physics (the mass of the dark matter) serves as a powerful discriminant against a variety of noise sources, enabling high precision searches for the ultra-weak effects of dark matter.

Even though there are a wide variety of theoretical dark matter candidates, there are only four dominant experimental signatures of this oscillating classical field. The oscillating field can induce currents in circuits, exert accelerations on test masses, cause precession of spins and change the values of fundamental constants. Current DOE OHEP supported experiments are searching for the first of these effects. With its unique sensitivity to accelerations, spin and atomic energy levels, MAGIS-100 would be sensitive to the three other dominant effects of dark matter in the mass range $10^{-22} \mathrm{eV}-10^{-15} \mathrm{eV}[6-8]$. In fact, three separate dark matter searches can be performed using this quantum test bed.

First, dark matter that affects fundamental constants, such as the electron mass or the fine structure constant, will change the energy levels of the quantum states used in the interferometer. This effect can be searched for by comparing two simultaneous atom interferometers separated along the MAGIS-100 baseline [6]. The sensitivity to several such dark matter candidates is shown in Figs. 1 and 2. Note that this search is highly sensitive in the frequency band from $0.1 \mathrm{~Hz}$ to $10 \mathrm{~Hz}$ for MAGIS-100. In the mass range $10^{-14} \mathrm{eV}<m_{\phi}<10^{-15} \mathrm{eV}$, MAGIS-100 would be expected to improve on existing bounds by up to two orders of magnitude.

Second, dark matter that causes accelerations can be searched for by comparing the accelerometer signals from two simultaneous quantum interferometers run with different isotopes ( $\mathrm{Sr}$ for example) [8]. This requires running a dual-species atom interferometer, which has already been 


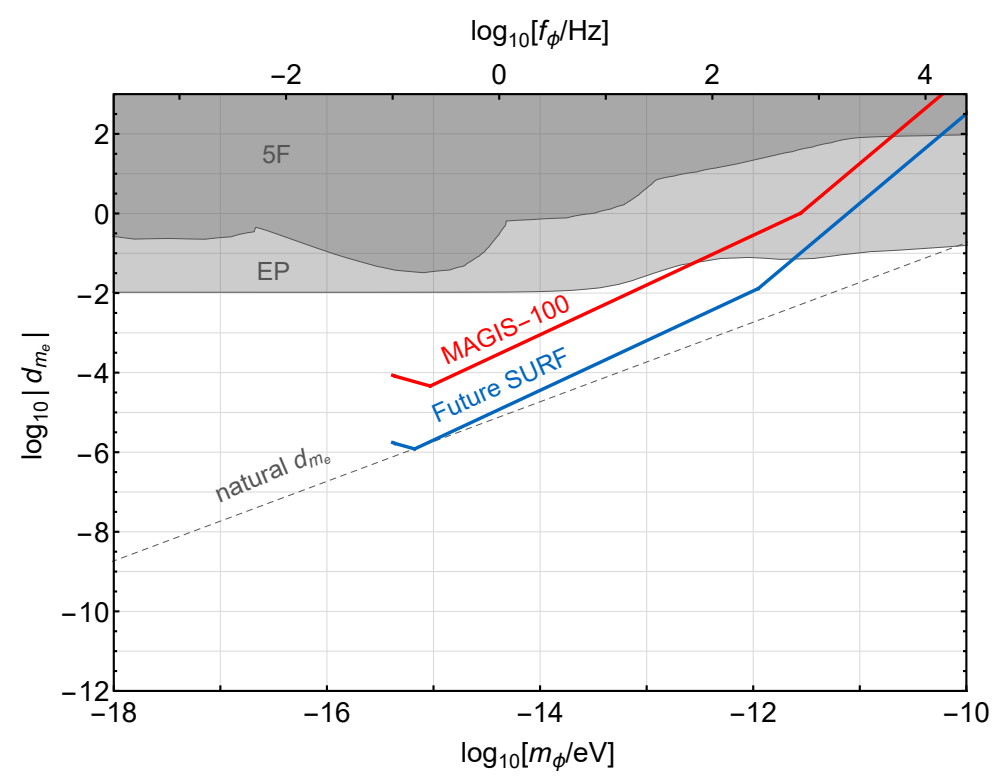

Figure 1: Sensitivity of MAGIS-100 to an ultralight dark matter field coupling to the electron mass with strength $d_{m_{e}}$, shown as a function of the mass of the scalar field $m_{\phi}$ (or alternatively the frequency of the field - top scale) [6]. The red curve, which shows the reach for an exposure of $10^{15}$ dropped atoms, assumes a shot-noise limited phase resolution and corresponds to about 1 year of data taking (1000 $\hbar k$ atom optics, $10^{-4} \mathrm{rad} / \sqrt{\mathrm{Hz}}$ phase resolution). The gray bands show existing bounds (derived from equivalence principle (EP) and fifth force $(5 \mathrm{~F})$ tests). The blue curve is the projected sensitivity of a future kilometer-scale detector at the SURF (Homestake) site.

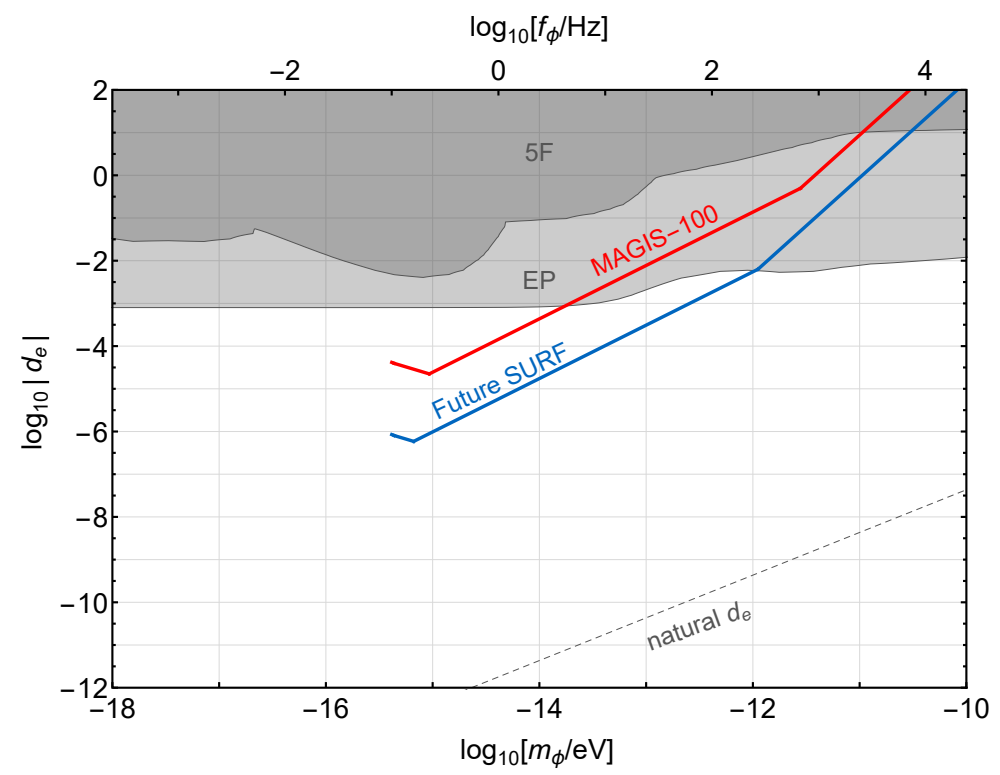

Figure 2: Sensitivity of MAGIS-100 to dark matter via coupling to the fine structure constant with strength $d_{e}$, shown as a function of the mass of the scalar field $m_{\phi}$ (or alternatively the frequency of the field - top scale) [6]. The red curve, which shows the reach for an exposure of $10^{15}$ dropped atoms, assumes a shot-noise limited phase resolution and corresponds to about 1 year of data taking (1000 $\hbar k$ atom optics, $10^{-4} \mathrm{rad} / \sqrt{\mathrm{Hz}}$ phase resolution). The gray bands show existing bounds (derived from equivalence principle (EP) and fifth force $(5 \mathrm{~F})$ tests). The blue curve is the projected sensitivity of a future kilometer-scale detector at the SURF (Homestake) site. 


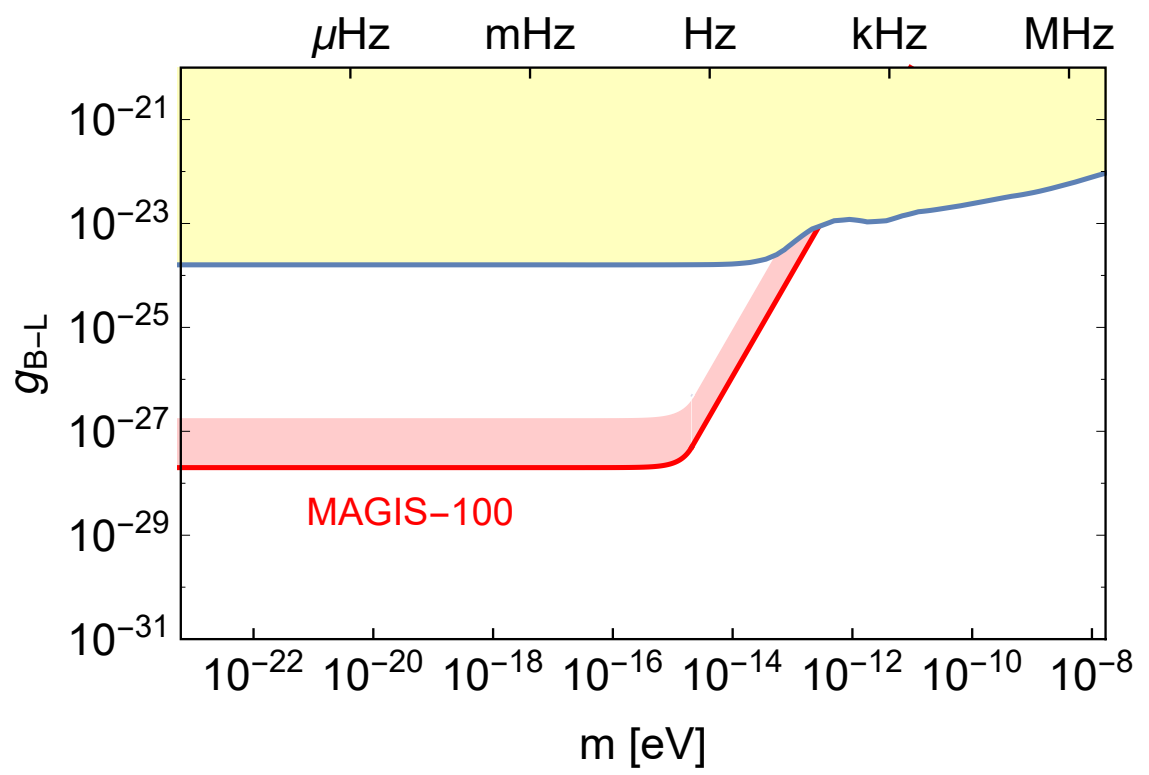

Figure 3: Sensitivity of MAGIS-100 to a B-L coupled new force, with $10^{-16} \mathrm{~g} / \sqrt{\mathrm{Hz}}$ acceleration sensitivity (assumes $50 \mathrm{~m}$ launch, $1000 \hbar k$ atom optics, $10^{8}$ atoms/s flux, shot noise limited). Shaded red band shows estimated uncertainty in projected sensitivity. Yellow band indicates existing bounds. Potential sensitivities of this method to general other dark matter candidates are shown in $[8]$.

demonstrated [9-12]. The potential sensitivity of MAGIS-100 to one such dark matter candidate, a B-L coupled new vector boson, is shown in Fig. 3. In general, potential sensitivities to dark matter candidates are shown in [8]. Note that, compared to existing bounds, MAGIS-100 would improve the sensitivity to any such dark matter particles with mass (frequency) below approximately $10^{-15} \mathrm{eV}(0.1 \mathrm{~Hz})$ by about two orders of magnitude.

Interestingly, the two dark matter searches described above are sensitive to similar dark matter candidates, but within complementary mass ranges, extending the coverage of the dark matter parameter space.

Third, dark matter that causes precession of nuclear spins, such as general axions, can be searched for by comparing simultaneous, co-located interferometers using Sr atoms in quantum states with differing nuclear spins. See [7] for a discussion and potential sensitivities.

\subsection{New Forces}

In addition to these dark matter searches, new fundamental particles may also be discovered by searching for new forces. This opportunity was identified in [2]. Ultra-light particles that have highly suppressed interactions with Standard Model particles, often dubbed "dark sectors", emerge in a variety of beyond-the-Standard-Model frameworks. These theories include forces mediated by particles that can dynamically solve naturalness problems in the Standard Model, such as the strong CP problem (QCD axion [13]) and the hierarchy problem (relaxion [14]). Such forces can also arise in theories with extra-dimensions [15] as well as super-symmetry [16]. Due to its high precision, MAGIS-100 can search for these ultra-weak forces, sourced either by the Earth or a test mass. Several of these particles have made an appearance in the previous subsection as ultra-light dark matter candidates. Here we can also search for the presence of these fields, but not necessarily as dark matter. In principle there are two ways to do this. First, if the range of the new force is short, it can be observed by modulating the distance between a test mass and the atomic sensor. 
Second, long range forces sourced by the Earth but not due to gravity may lead to differential free-fall accelerations between different elements/isotopes. A comparison between atomic sensors made out of different elements/isotopes could reveal the existence of such forces. MAGIS-100 can perform such a test by performing simultaneous acceleration measurements with two isotopes of Sr.

\subsection{Quantum Science}

The key role that could be played by quantum technologies in a variety of scientific applications has also been recognized in several DOE community reports $[2,3]$. These reports have identified grand challenges and opportunities in the field of quantum information science where strategic investments could lead to significant payoffs. MAGIS-100 would demonstrate key quantum technologies that promise to achieve these grand community challenges.

The initial focus of MAGIS-100 is to successfully deploy a quantum sensor network with two nodes, where each node consists of an atom interferometer. The quantum information, stored as a phase in each sensor, is compared across a long baseline. The operation of this network requires the ability to coherently manipulate each sensor, thus serving as a testbed for quantum control protocols. MAGIS-100 would take advantage of recent advances $[1,17]$ in manipulation of atoms using light, as well as long free-fall times, to realize macroscopic quantum mechanical superposition states. In MAGIS-100, atom de Broglie wave packets are expected to be separated by distances of up to $10 \mathrm{~m}$. Such quantum superposition states are delocalized on a truly macroscopic scale and can be described as a state with an atom located in two places at once. By operating two or more such interferometers simultaneously and comparing their interference patterns, the MAGIS-100 Collaboration will discover whether the coherence of the macroscopic quantum superposition can be maintained at such large length scales. The MAGIS-100 instrument will also support recordbreaking matter wave interferometer durations, up to 9 seconds for a full height launch.

The goal of MAGIS-100 is to operate this quantum sensor network with two nodes at the quantum limit, creating a testbed that will demonstrate the principles of quantum mechanics on unprecedented macroscopic time $(\sim$ seconds) and length scales $(\sim 100 \mathrm{~m})$. The sensor design takes advantage of features used by the best atomic clocks and combines them with established techniques for building inertial sensors based on atom interferometry. This testbed can also incorporate entangled quantum sources to reduce noise, permitting enhanced sensitivity. Spin squeezed atom sources $[18,19]$ take advantage of quantum correlations within an atom ensemble to realize a reduction in sensor noise below the standard quantum limit (shot noise). The MAGIS-100 Collaboration aims to study this using quantum entanglement as a resource in order to improve sensitivity by one to two orders of magnitude.

Successful implementation of MAGIS-100 will thus create a quantum sensor network using ultra-cold atoms that could enable a variety of scientific applications. It can be immediately used to test conventional quantum theory and potentially search for non-linear corrections to the Schrödinger equation, a need identified in [3]. Moreover, the quantum control over ultra-cold atoms demonstrated by MAGIS-100 could serve as a launching pad for future applications of ultra-cold atomic sensors in quantum simulations, particularly for dynamical gauge fields [3].

\subsection{Gravitational Waves}

The recent discovery of gravitational waves by LIGO is a historic event. It is a re-affirmation of general relativity and, more importantly, by allowing observations that are impossible with normal electromagnetic telescopes, it gives us a new way to study the universe. In addition, in contrast 
to photons which are thermalized prior to the time of CMB formation, gravitational waves do not thermalize and can carry information about the earliest epochs in the universe, back to and even including inflation. Since the early universe was very hot, observing these early times also teaches us about physics at the highest energies.

MAGIS-100 is not expected to be sufficiently sensitive to detect known candidate sources of gravitational waves. Nevertheless, within its frequency range it would have a record sensitivity, improving on the current bounds [20] by many orders of magnitude (Fig. 4). Hence, there is always the possibility of an unexpected discovery. In addition, MAGIS-100 would provide a critical step towards a longer km-scale experiment (MAGIS-1000, see Appendix G) which we anticipate would achieve sufficient sensitivity to measure gravitational waves from known sources. Gravitational wave measurements are therefore an important part of the motivation for the MAGIS program.

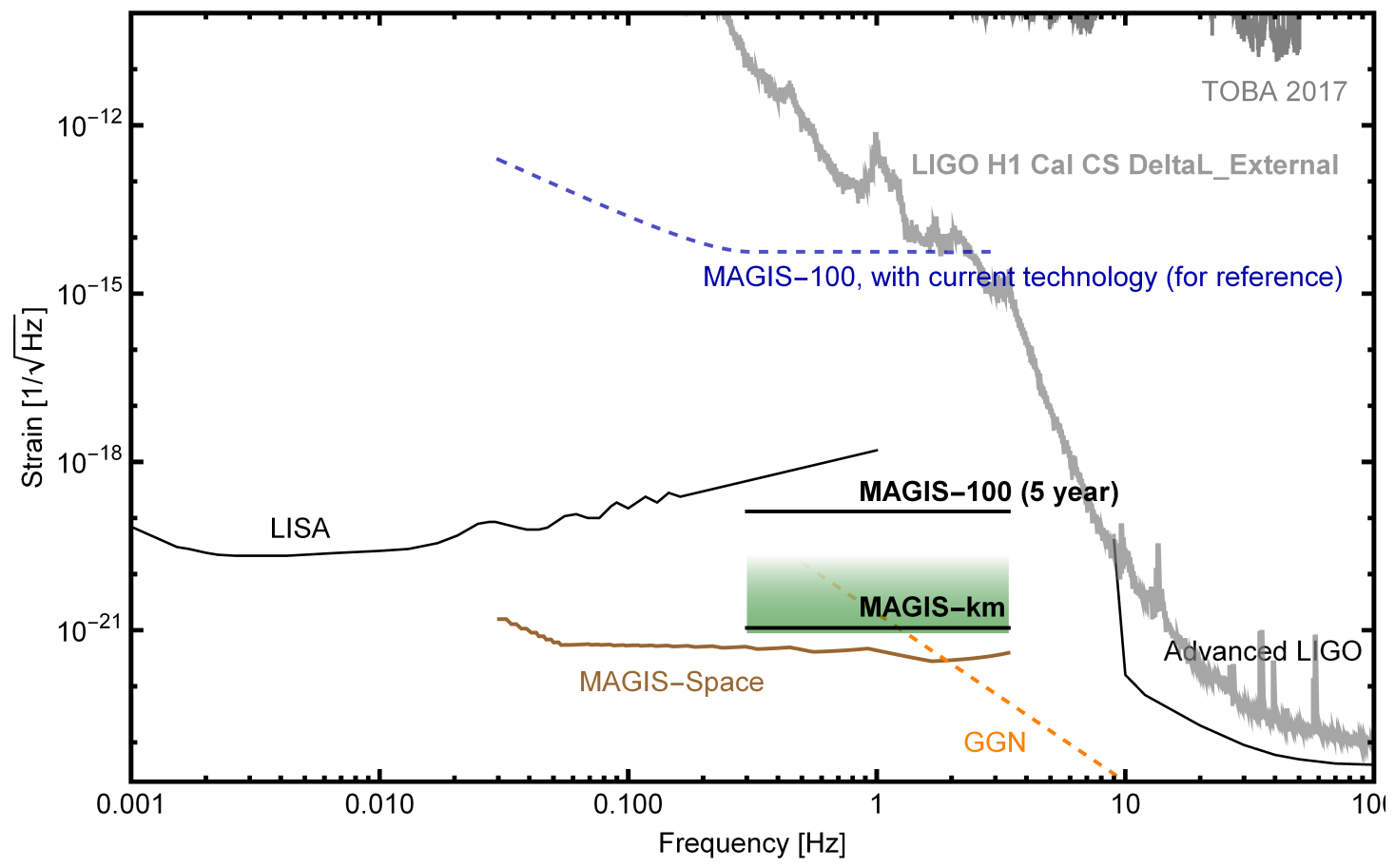

Figure 4: Gravitational wave sensitivity, characterized by the strain sensitivity, for MAGIS-100 shown as a function of frequency. The initial curve (blue-dash) shows the strain sensitivities of the $100 \mathrm{~m}$ detector to be located at Fermilab using current state of the art parameters (100 $\hbar k$, $\delta \phi=10^{-3} \mathrm{rad} / \sqrt{\mathrm{Hz}}$ ). The black curve (labelled MAGIS-100 5 year) shows what is possible after sensor research and development (see Section 8) and further pushing large momentum beam splitters to their physical limits $\left(4 \times 10^{4} \hbar k, \delta \phi=10^{-3} \mathrm{rad} / \sqrt{\mathrm{Hz}}\right)$. Also shown is the estimated sensitivity of a future km-scale experiment. Existing limits from TOBA [20] are shown in gray. LIGO and LISA sensitivity curves are shown for reference. A preliminary estimate of Newtonian gravitational gradient noise (GGN, see Appendix I), which is expected to limit future terrestrial detectors at low frequencies, is shown in orange.

The prospect of using atom interferometers and atomic clocks for gravitational wave detection has been extensively studied [21-27]. Passing gravitational waves cause a small modulation in the distance between objects. Detecting this modulation requires two ingredients:

1. Inertial references: A pair of freely-falling objects, separated by some distance (the 'base- 
line'), to act as inertial reference points. Good inertial reference objects must be largely immune to perturbations from non-gravitational forces so that the primary source of any modulation in the baseline is due to the gravitational wave.

2. Clock: A means of precisely measuring the separation between the inertial references. This is typically done by measuring the time for light to cross the baseline, so this amounts to requiring a very precise clock.

The MAGIS concept [28], allows a single baseline gravitational wave detector [23, 29,30] to be realized. Dilute clouds of ultracold atoms at both ends of the baseline act both as inertial references and as clocks. MAGIS will use Sr atoms which act as excellent clocks [31]. Laser light propagates between the two atom-ensembles and interacts with the atoms, driving transitions between the ground and excited atomic clock levels. The timing of these transitions depends on the light travel time across the baseline. A passing gravitational wave can therefore result in a shift of the atomic state [23]. It is worth noting that the numbers characterizing the stabilities of state-ofthe-art atomic clocks $[31,32]$ are not directly applicable to the MAGIS concept. This is because the MAGIS sensitivity is dramatically enhanced by large momentum transfer atom optics (see Sec. 3.4), and the long interferometer lengths for MAGIS allow increased interrogation time. Moreover, the atoms in MAGIS are freely falling rather than confined to an optical lattice, removing the effects of vibrational noise [23].

Note that LIGO and other ground-based laser interferometer designs are sensitive to gravitational waves between about $10 \mathrm{~Hz}$ and $1 \mathrm{kHz}$, but are severely limited at lower frequencies due to seismic noise. At much lower frequencies, the proposed LISA detector is targeted at the $1 \mathrm{mHz}^{-}$ $50 \mathrm{mHz}$ range. MAGIS-1000 promises to cover the "mid-band" gap between LIGO and LISA, roughly $30 \mathrm{mHz}-10 \mathrm{~Hz}$ (see Fig. 4). The gravitational wave events that have been detected so far indicate that strain sensitivities of $10^{-20} / \sqrt{\mathrm{Hz}}$ in the mid-band should enable measurements of known sources (the black hole binaries detected by LIGO). It is quite possible that there are new sources that are above $10^{-20} / \sqrt{\mathrm{Hz}}$ in this band, and that have remained undetected because they do not make it all the way to LIGO's band before merging (e.g., heavier black hole binaries). The sensitivity of the km-scale MAGIS is $10^{-21} / \sqrt{\mathrm{Hz}}$, an order of magnitude below this threshold.

The mid-band may be optimal for observing the highest energy scales in the very early universe. It is above the white dwarf "confusion noise" but can still be low enough in frequency to see certain cosmological sources. This band can be an excellent place to search for gravitational waves from inflation and reheating, and certain models such as axion inflation may also give signals large enough to be detected by future versions of MAGIS.

Additionally, detectable gravitational wave signals in this band may be produced by phase transitions in the early universe at scales above the weak scale, networks of cosmic strings, or mergers of black holes that are too heavy for LIGO to detect (hundreds of solar masses or above).

Such unknown sources would clearly be a major discovery, but there are also important sources in the mid-band which are certain to exist, for example neutron star binaries and black hole binaries with masses around few to tens of solar masses. Many black hole or neutron star binaries observed in this band could later be observed by LIGO once they pass into the higher frequencies. Such joint observation would be a powerful new source of information. For example, an atomic detector could give a prediction of the time and location of a merger event. Since the sources generally live a long time in this mid-frequency band, they can be localized on the sky even by a single-baseline detector and in fact the mid-band is ideal for localization and prediction of merger events [33].

There are also white dwarf binary mergers in this band, which are not observable at higher frequencies. Such a merger could be a type IA supernova. The question of the origin of type 
IA supernovae, whether from a single neutron star or a binary merger, has attracted significant interest and is clearly of major importance (see e.g. [34] and references therein). As just one example the nature of type IA supernova clearly affects their use as standard candles for measuring the cosmological expansion rate and the properties of dark energy.

To better understand the nature and origin of black holes, including those being observed by LIGO, it would be very beneficial to have a measurement of their initial spins and orbital eccentricity. This can for example discriminate between different production mechanisms (e.g. cosmological or astrophysical) [34]. To measure the initial spins with great accuracy it is necessary to observe not just the merger, but also many pre-merger cycles. Thus, the mid-band appears to be a promising band for measuring the spin of merging black holes, including the mergers seen by LIGO.

Observing compact objects such as black holes, neutron stars, and white dwarfs is of course astrophysically interesting and important. Additionally, such objects may well teach us about particle physics. For example, supernovae and other such extreme astrophysical objects have already been used to set some of the best limits on axions and other light particles and gravitational wave observations may allow many more such tests for new physics. As just one example, superradiance around black holes (e.g. [35]) may allow us to constrain or even discover such particles with future gravitational wave observations. To use black hole superradiance for axions we must have precise measurements of the black holes' initial spins. As noted above, the mid-band appears quite promising for these black hole spin measurements.

Finally, such astrophysical observations can shed light on important cosmological questions. It has long been known that a binary merger can be a gravitational wave "standard siren" [36]. Such standard sirens allow us to measure the expansion rate of the universe with fewer systematic uncertainties because the gravitational merger signal is very clean. Accurate angular localization is very important for this measurement and thus MAGIS can contribute significantly to this program [37]. This will enable a better understanding of cosmology, in particular a better measurement of the Hubble constant, and the dark energy equation of state.

\section{Experimental Overview}

\subsection{A light pulse atom interferometer}

A light pulse atom interferometer consists of the following ingredients:

- A cold atom source. Atoms are collected and cooled in a multi-step process involving laser cooling, evaporative cooling, and delta kick cooling. The cooling is needed to reduce the velocity spread (temperature) of the ensemble and hence limit the transverse spreading of the atoms during the long interferometry times. This is required to enable efficient light-atom interactions. To maximize sensitivity, it is important that the cooled ensembles have as many atoms as possible. The atoms are prepared so that they are all in the ground state.

- An atom cloud shuttle. The cooled atom cloud is transferred from the atom source to the adjoining interferometry region (vertical pipe). This is implemented using an optical dipole trapping potential and an optical lattice to support the atoms against gravity and accelerate them from the source chamber to the interferometer chamber.

- A launch system. Once in the vertical interferometer pipe, the atom ensembles are launched vertically or dropped, after which they begin to follow free-fall trajectories. The launch is implemented by a vertical optical lattice potential. 
- A long vertical pipe. This provides the environment in which the atoms fall under gravity. The pipe is evacuated and shielded from external magnetic fields. Within the shield, one or more coils produce a small bias field, which defines a reference direction for the atomic spins.

- A laser system to create a light pulse atom interferometer. Once the atoms are falling, a series of laser pulses are sent along the vertical axis of the pipe. These light pulses act as beamsplitters and mirrors for the matter waves.

- An atom detection system. The matter wave interference pattern is imaged using a camera. This occurs in special detection regions along the main interferometry pipe. The detection regions have vacuum viewports to allow optical access for imaging. The images are formed by shining resonant light on the atoms and then collecting the resulting fluorescence.

Laser pulses are used to drive the interferometer. A light pulse can transfer momentum $\hbar k$ to the atom, where $k$ is the wavevector of the light, as well as flip the atomic internal state. For example, the atom while in state $|1\rangle$ can absorb a photon, causing it to transition to state $|2\rangle$ and recoil with additional velocity $v_{r}=\hbar k / m$, the 'recoil velocity'. The reverse process can also occur in which the atom undergoes stimulated emission by emitting a photon, slowing down by the recoil velocity and transitioning from state $|2\rangle$ to $|1\rangle$. By using precise control of the frequency and duration of the light pulses, it is possible to continuously tune the probability of making such a transition ${ }^{2}$. A beamsplitter pulse (' $\pi / 2$ pulse') is one which leaves the atom in an equal superposition of states $|1\rangle$ and $|2\rangle$. A mirror pulse (' $\pi$ pulse') is one which flips the states $(|1\rangle \leftrightarrow|2\rangle$ ) by emission or absorption of a photon.

Figure 5 shows a space-time diagram that illustrates how a sequence of three of these laser pulses can be used to create an interferometer. Consider a two-state atom that has been prepared in its ground state and is dropping under gravity at the top of the pipe. Here it interacts with the first laser pulse at time $t=0$. The frequency, intensity and duration of this "beamsplitter" pulse are chosen so that the atom ends up in an equal superposition of the ground and excited states. Its wavefunction now has two pieces. The piece that describes the excited state corresponds to an atom that has absorbed a photon, and hence absorbed the photon's momentum. Hence the two parts of the wavefunction describe states falling with slightly different velocities and with slightly different de Broglie wavelengths. As the atom falls, a phase difference builds up between these two wavepackets. At time $T$, the atom interacts with a second laser pulse. The frequency, intensity and duration of this "mirror" pulse are chosen to change the state of the atom (ground $\leftrightarrow$ excited) with the corresponding absorption or emission of a photon. The two wavepackets that describe the atom now have their momenta interchanged and, as the atom continues to fall, they begin to come together. At time $2 T$ the wavepackets will once again overlap, and at this time the atom interacts with a third "beamsplitter" laser pulse. The atomic wavefunction after this third pulse will have an interference term which depends upon the phase difference acquired by the two wavepackets as they traveled along their separate paths. A light pulse atom interferometer can be used to search for any new physics that modifies the phase difference.

\section{$3.2 \quad$ A Gradiometer}

The phase difference measured by a single interferometer of the type described above will have a contribution from laser noise, arising from the intrinsic instability of the laser and from vibration of the delivery optics. The gradiometer uses two identical interferometers separated by a large distance, and run simultaneously using common laser pulses (Fig. 6). The phase shifts measured by

\footnotetext{
${ }^{2}$ In the presence of the light the atom undergoes Rabi oscillations.
} 


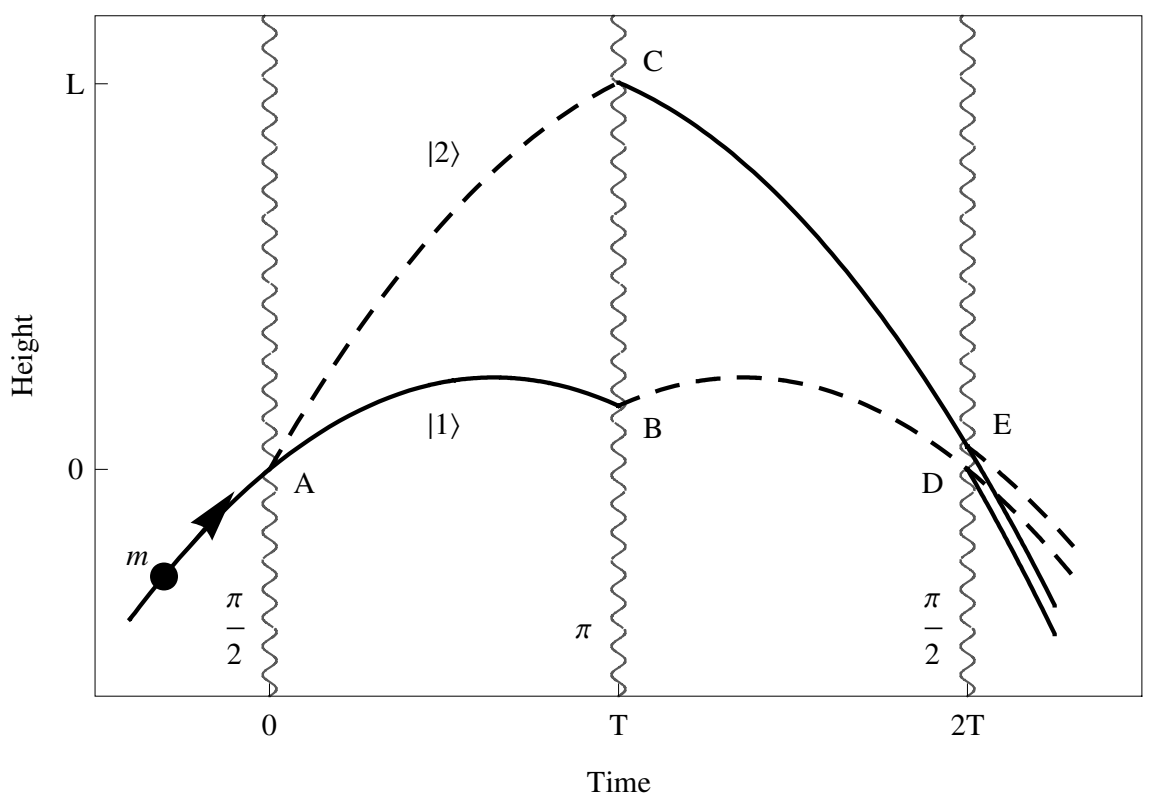

Figure 5: Light pulse atom interferometer. The atom has mass $m$ and is manipulated by a series of three short laser pulses (wavy lines). The light transfers momentum to the atom and toggles the internal state between $|1\rangle$ and $|2\rangle$. The pulses can be configured as beamsplitters $(\pi / 2$ pulse area) or mirrors ( $\pi$ pulse area). Together, the three-pulses split, redirect, and recombine the atomic wavepackets, yielding a matter wave interference pattern. The classic $\pi / 2-\pi-\pi / 2$ sequence shown here results in a Mach-Zehnder geometry and is sensitive to accelerations. More complex sequences are possible using additional pulses.

the two interferometers are compared, and this differential signal is used to search for new physics. Note that the differential measurement enables the cancellation of noise common to both interferometers [23], for example the laser noise. Common mode noise cancellation using a gradiometer configuration has been demonstrated [1] at Stanford, and previously in many other experiments such as [38]. Note also that the phase difference recorded by each interferometer depends on the time spent in the excited state, which is directly tied to the light travel time $(L / c)$ across the baseline as well as the internal energy difference between the energy states. Ultralight dark matter can be detected because it modifies the clock energy levels. Gravitational waves can be detected because they modulate the light travel time.

\subsection{Choice of atomic species}

Various atomic species have been used in light pulse atom interferometers. Typically, alkali atoms are used ( $\mathrm{Rb}$ or Cs being two popular candidates). Instead, MAGIS-100 will use group II (alkalineearth) atoms, specifically strontium (Sr). The most precise atomic clocks in the world are now made using Sr [31,39], and MAGIS-100 will take advantage of the advances in this field. Critically, a gradiometer based on $\mathrm{Sr}$ can be made intrinsically insensitive to laser frequency noise, an important element of the detector noise budget. This is because Sr atoms allow for a new method of implementing the atomic beam splitters and mirrors (the "atom optics") that make up the interferometer. Traditional alkali atom interferometers use atom optics that are based on two-photon Raman (or Bragg) transitions that must be driven by two counter-propagating laser beams [40]. However, laser frequency noise does not exactly cancel in a gradiometer that uses counter-propagating beams due to asymmetry in the light travel times to the atoms [23]. By contrast, Sr atoms possess a 
(a)

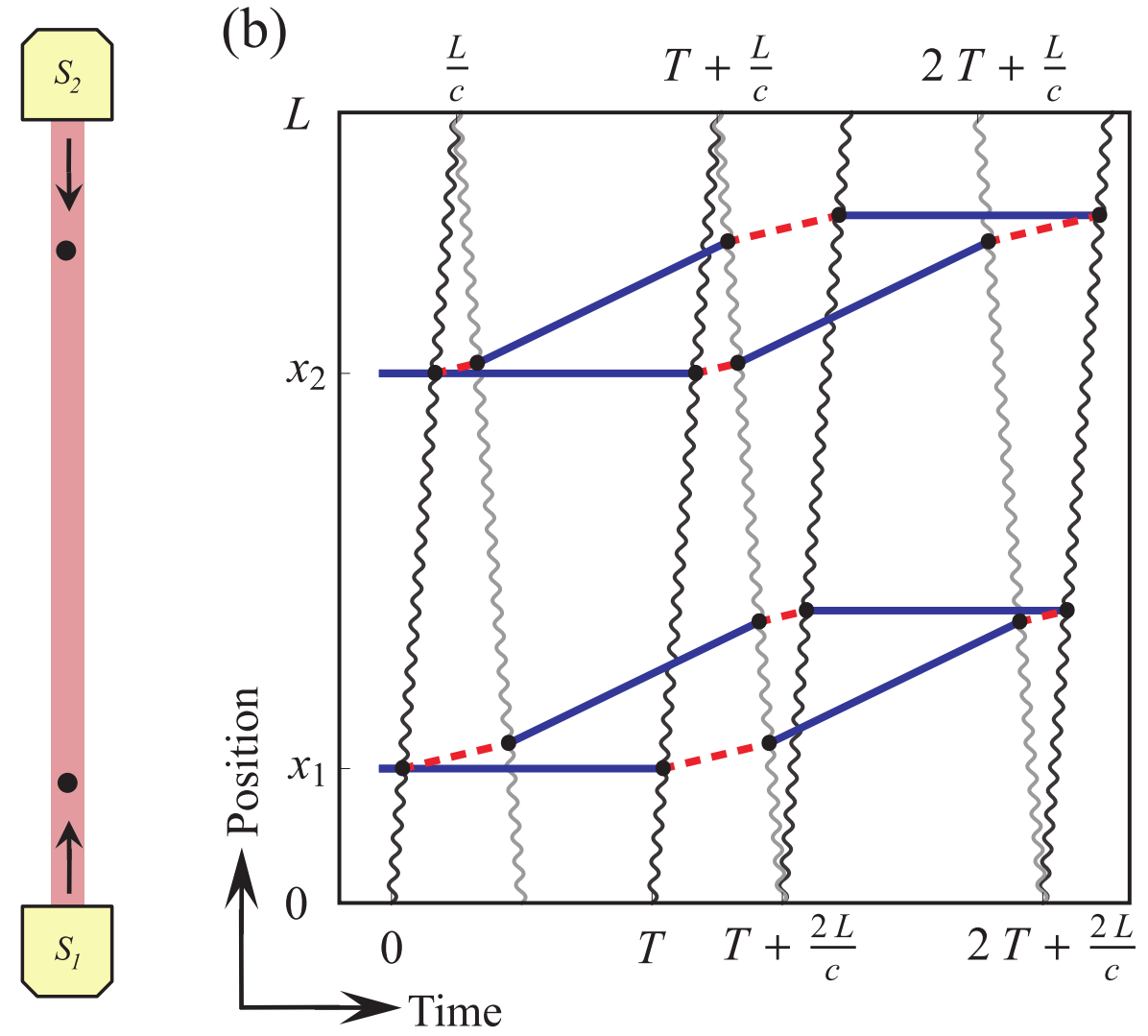

Figure 6: A Gradiometer. (a) Dilute clouds of atoms (black circles) at either end of a long baseline act as inertial test masses. Laser light (red) propagates between the atoms from sources $S_{1}$ and $S_{2}$. The light is used to implement atom interferometry locally at each end of the baseline. The resulting interference patterns are compared. The phase difference between the two interferometers results in the gradiometer signal, which is sensitive to the relative acceleration between the atoms and any differential clock phase evolution. (b) Space-time diagram of the trajectories of two atom interferometers based on single-photon transitions, showing the ground (blue) and excited (red dashed) states. The atoms are initially placed at positions $x_{1}$ and $x_{2}$, corresponding to opposite ends of the baseline (i.e. the black circles in (a).) Short laser pulses (wavy lines) traveling from alternating sides of the baseline are used to divide, redirect, and recombine the atom de Broglie waves. For simplicity, the effect of gravity on the trajectories is not shown. 
narrow transition to a long-lived excited state which only requires a single laser beam to excite. Implementing an atom interferometer using these single-photon transitions in group II atoms like $\mathrm{Sr}$ allows for superior common-mode rejection of laser frequency noise compared to what is currently possible with two-photon transitions [23]. This method of atom optics cannot be used in alkali atoms because the optically excited state in these atoms is very short lived.

Another important advantage of $\mathrm{Sr}$ atoms is significant reduction of sensitivity to magnetic fields. Alkali interferometers use magnetically insensitive $m=0$ states to minimize the effect of magnetic forces on the atoms. However, these states still display a second order Zeeman energy shift which has size $\sim \mathrm{kHz} /$ gauss $^{2}$, requiring magnetic field control below the milligauss level for optimal performance. The magnetic susceptibility of the clock transition in strontium atoms is approximately 1000 times smaller $^{3}$ than in the alkali metals, on the order of $\mathrm{Hz} /$ gauss $^{2}$ [41]. This dramatically reduces the requirements on the magnetic shield.

\subsection{Using advanced atom optics}

The pulse sequences used to drive the interferometer can be modified to enhance the sensitivity of the detector. Two advanced atom optics techniques are shown in Fig. 7. First, the sensitivity can be enhanced linearly by increasing the number of light pulses associated with each atom optic by a factor of $n$. Figure 7 (left) shows an example of such a large momentum transfer (LMT) atom optic, where $(n-1)$ additional $\pi$-pulses are appended to the initial beamsplitter pulse. These additional pulses increase the momentum transferred to one arm of the interferometer, leading to increased wavepacket separation.

The sensitivity can also be enhanced by using a multi-loop sequence, as shown in Fig. 7 (right). In such a sequence, the atomic wavepackets oscillate back and forth with some chosen period $T$, leading to a resonant enhancement of the sensitivity of the detector at frequency $\sim 1 / T[25]$. The interferometer can be run in a resonant mode by using the pulse sequence $\pi / 2-\pi-\cdots-\pi-\pi / 2$ with $Q \pi$ pulses instead of the standard, broadband $\pi / 2-\pi-\pi / 2$ pulse sequence described before. For signals that oscillate in time, the phase differences accumulated during each loop of the interferometer continually add in the resonant sequence, as long as the period of the signal is matched to the interferometer period. As a result, the enhancement in a resonant sequence comes at the cost of bandwidth, so detection strategies must be used that appropriately adjust the pulse sequence to scan the detector sensitivity. Resonant enhancement can also be combined with LMT atom optics by replacing each pulse in the resonant sequence with an LMT-enhanced version.

MAGIS-100 will provide a test bed for developing these advanced optics techniques to enhance the sensitivity of the next generation experiment (MAGIS-1000, see appendix C).

\subsection{Using three atom sources}

The proposed MAGIS-100 setup uses three atom sources, located at the top, middle, and bottom of the vertical shaft (see Fig. 8). Using three sources allows for flexible operation of the detector. In one mode, atoms from the upper and middle sources are dropped simultaneously and are allowed to fall $50 \mathrm{~m}$ before being detected. This mode allows for a long free-fall time $(>3 \mathrm{~s})$ to facilitate low frequency operation while maintaining a $50 \mathrm{~m}$ baseline (separation between atoms). In another mode, atoms from the bottom and upper source are simultaneously launched upward by $10 \mathrm{~m}$ (with an optical lattice) and then fall back down to the original position of the source where they are detected. This mode maximizes the baseline $(\sim 100 \mathrm{~m})$ while still allowing for detection

\footnotetext{
${ }^{3}$ The fermionic isotope has an additional linear susceptibility, but this can be canceled by interrogating atoms with both positive and negative spin projection.
} 

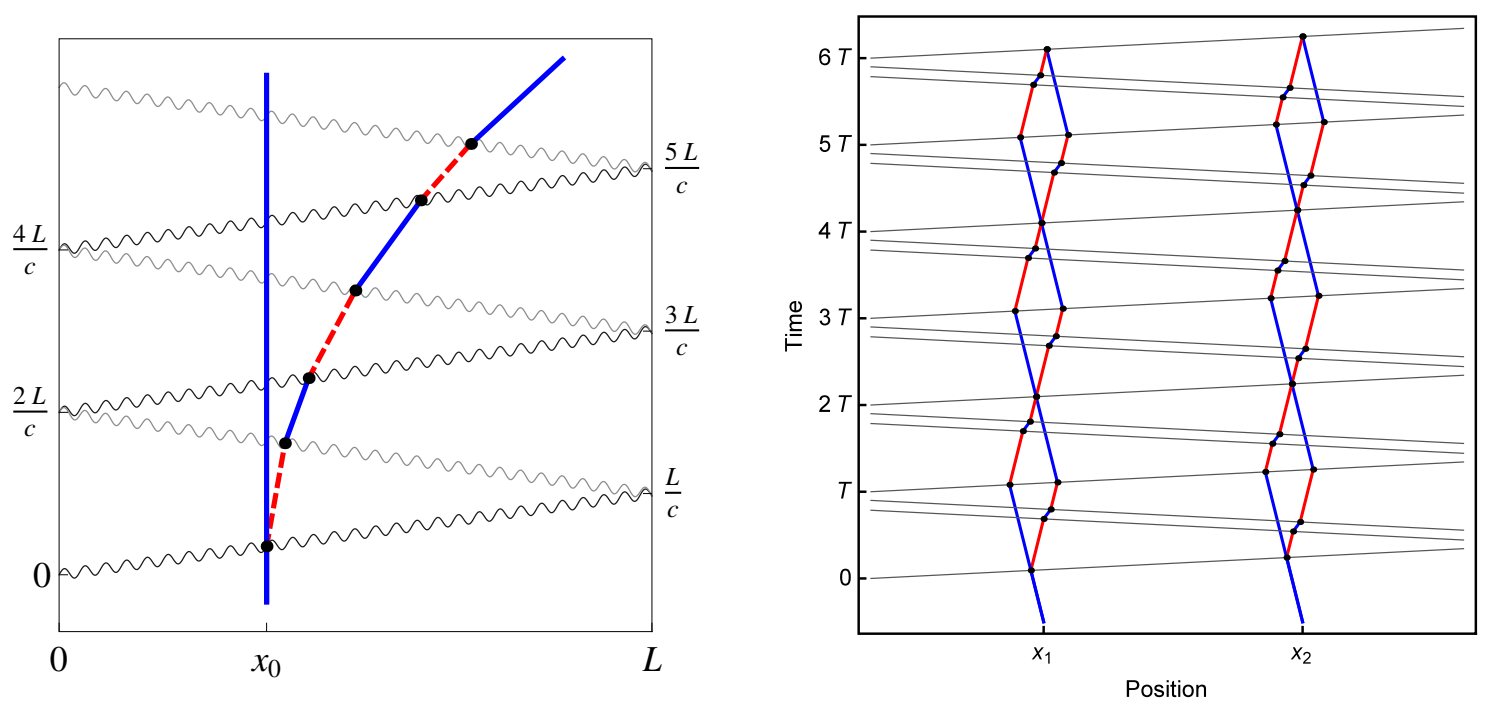

Figure 7: Advanced atom optics. The space-time trajectories of the atoms are shown in blue for the ground state and red for the excited state. Black dots indicate the vertices at which the laser interacts with the atom, as determined by Doppler shifts of the atomic resonance. Left: Space-time diagram of a large momentum transfer (LMT) beamsplitter with $N=3$ implemented using single photon transitions. Light pulses from the lasers are incident from the left (dark gray) and the right (light gray) respectively. Right: Space-time diagram showing resonant atom interferometry with $Q=3$ (three diamonds) in the MAGIS gradiometer configuration. Pulses of light (thin black lines) are sent back and forth from each end of the baseline and interact with the atoms on each end.

at frequencies below around $1 \mathrm{~Hz}(\sim 1 \mathrm{~s}$ free-fall time). All three sources can also be operated simultaneously (either with a short launch or drop). Having more than two sources distributed along the baseline is expected to help characterize and suppress gravity gradient noise [42] (see Appendix I) that otherwise limits any earth-based detector at low frequency (see Fig. 4). Gravity gradient noise is caused by the gravitational coupling of moving environmental masses (due to seismic motion of the earth) to the atomic test masses. A distributed array of sensors can potentially distinguish this noise from a signal by observing the spatial profile across the baseline, which is expected to be nonuniform for noise and, for example, uniform for a gravitational wave. MAGIS-100 will evaluate this concept.

\section{Present generation (10 m-scale) experiment \& illustrative re- sults}

Atom interferometers were first demonstrated in the early 1990's [43]. Examples of existing atom interferometers are given in [44]. Atom interferometry has been used to make precision inertial sensors, including gyroscopes [45], gradiometers [38], gravimeters [46], as well as precision measurements of fundamental constants such as Newton's constant [47-49] and the fine structure constant [50-52]. Supported by advances in cooling techniques that can now produce atom clouds in the picokelvin temperature range [53], atom interferometers have been shown to operate over durations exceeding $2 \mathrm{~s}$ [54], in a $10 \mathrm{~m}$ scale drop tower, limited by the available free-fall distance. Taking advantage of improvements in the ability to coherently transfer large amounts of momentum using light $[55,56]$, atoms have been split quantum mechanically into superposition states with wave-packet separations exceeding $50 \mathrm{~cm}$ [17] and subsequently interfered. Other notable achievements of the present generation of experiments include the operation of a high-accuracy absolute 

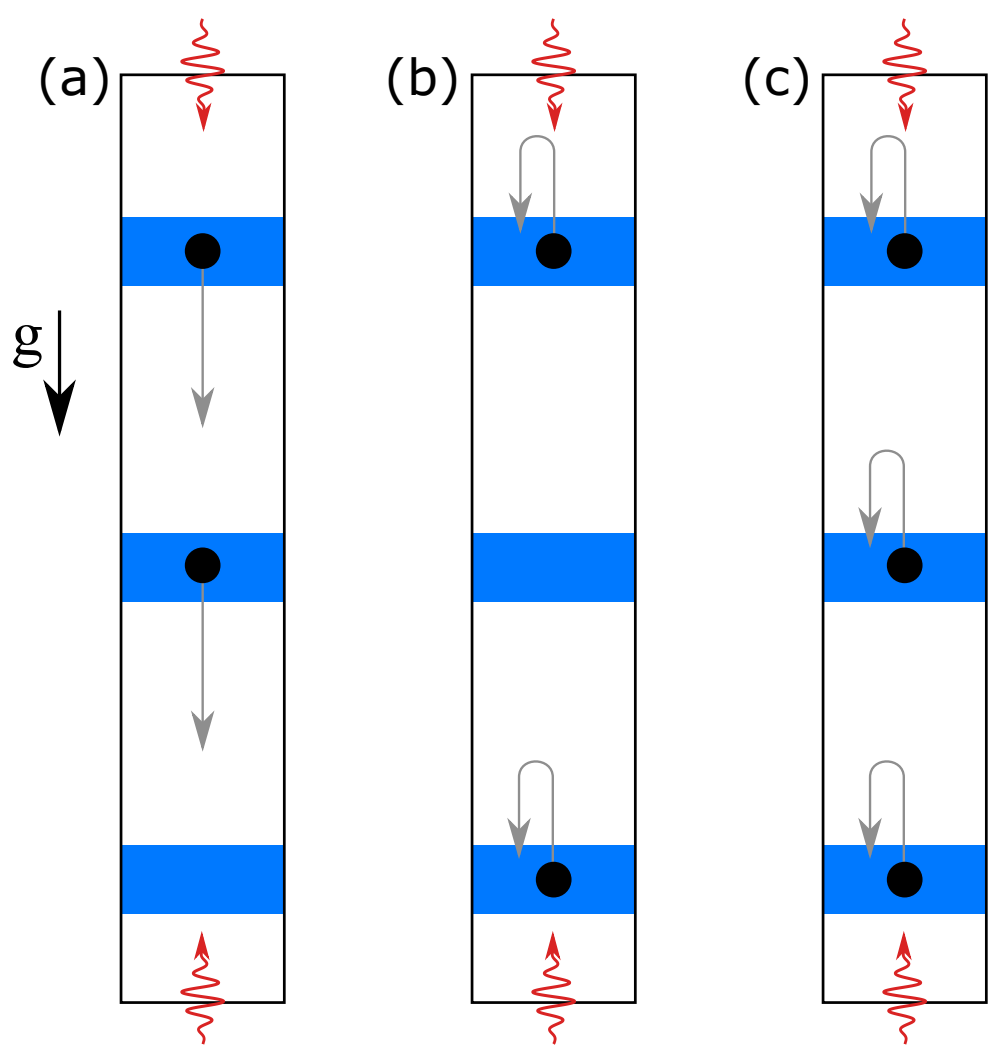

Figure 8: The basic MAGIS-100 detector design consists of three atom sources (blue bands) placed along the vacuum pipe at the top, middle, and bottom. Light pulses (red) travel along the vacuum pipe and interact with atoms at each of these locations. (a) Maximum drop time gradiometer. Atoms from the top and middle sources are dropped $50 \mathrm{~m}$ and detected at the middle at bottom locations, respectively. (b) Maximum baseline gradiometer. Atoms from the top and bottom sources are launched on short $(\sim 10 \mathrm{~m})$ trajectories and detected at the top and bottom. (c) GGN characterization. All three sources can be used with short launches in order to explore Newtonian noise variation along the baseline. 
gravimeter [57], which has the ability to clearly resolve time variations in local $g$ due to the tides, and enables the study of many systematic effects both theoretically and experimentally.

The theory and modeling of atom interferometers, and the general methods for calculating the interferometer phase shifts, have become well-established (see, for example, $[58,59]$ ). Note that, in general, phase shift calculations agree well with experimental measurements. Confidence in the measurements, and their interpretation, is obtained by varying experimental parameters (for example, baseline, launch velocity, laser pulse frequencies and durations) and comparing analytical predictions for the phase shifts with the data. This provides a powerful tool for separating signals from systematic effects, and for measuring the systematics.

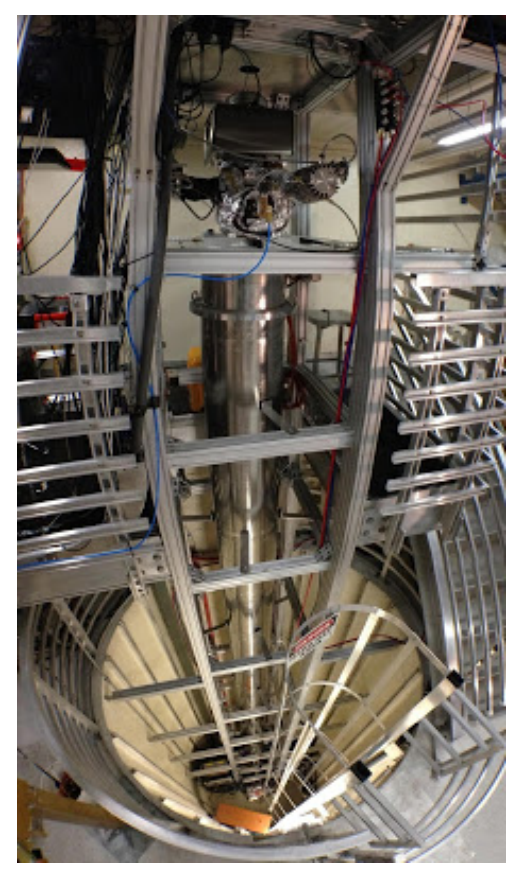

Figure 9: The current $10 \mathrm{~m}$ interferometer at Stanford. Showing the interferometer vacuum pipe, support structure, and the enclosure for the associated atom-optics at the top of the experiment.

\subsection{Results from the Stanford Experiment, a $10 \mathrm{~m}$ Fountain}

The proposed MAGIS-100 experiment is a natural extension of the existing experiment at Stanford University, shown in Fig. 9. To illustrate the power of the experimental technique, the following describes some representative results from the Stanford experiment.

- Measurement of the gravitational gradient produced by a test mass [1]. The atoms were launched upwards in a fountain configuration, and a removable test mass consisting of 7 lead bricks $(84 \mathrm{~kg}$ ) was positioned close to the top of the fountain. The bricks produced a phase shift of 1.0 radians, in agreement with predictions calculated numerically. The launch velocity of the atoms, and hence the height of the fountain, was then varied, and the observed phase shifts induced by the gravitational gradient produced by the test mass were found to be in good agreement with predictions (see Fig. 10).

- Using macroscopic quantum states [1] to observe the influence of space-time curvature across a single quantum system. The sensitivity of the interferometer was enhanced using macroscopic quantum states with spatial delocalization of up to $16 \mathrm{~cm}$ for $1 \mathrm{~s}$ time scales. These 

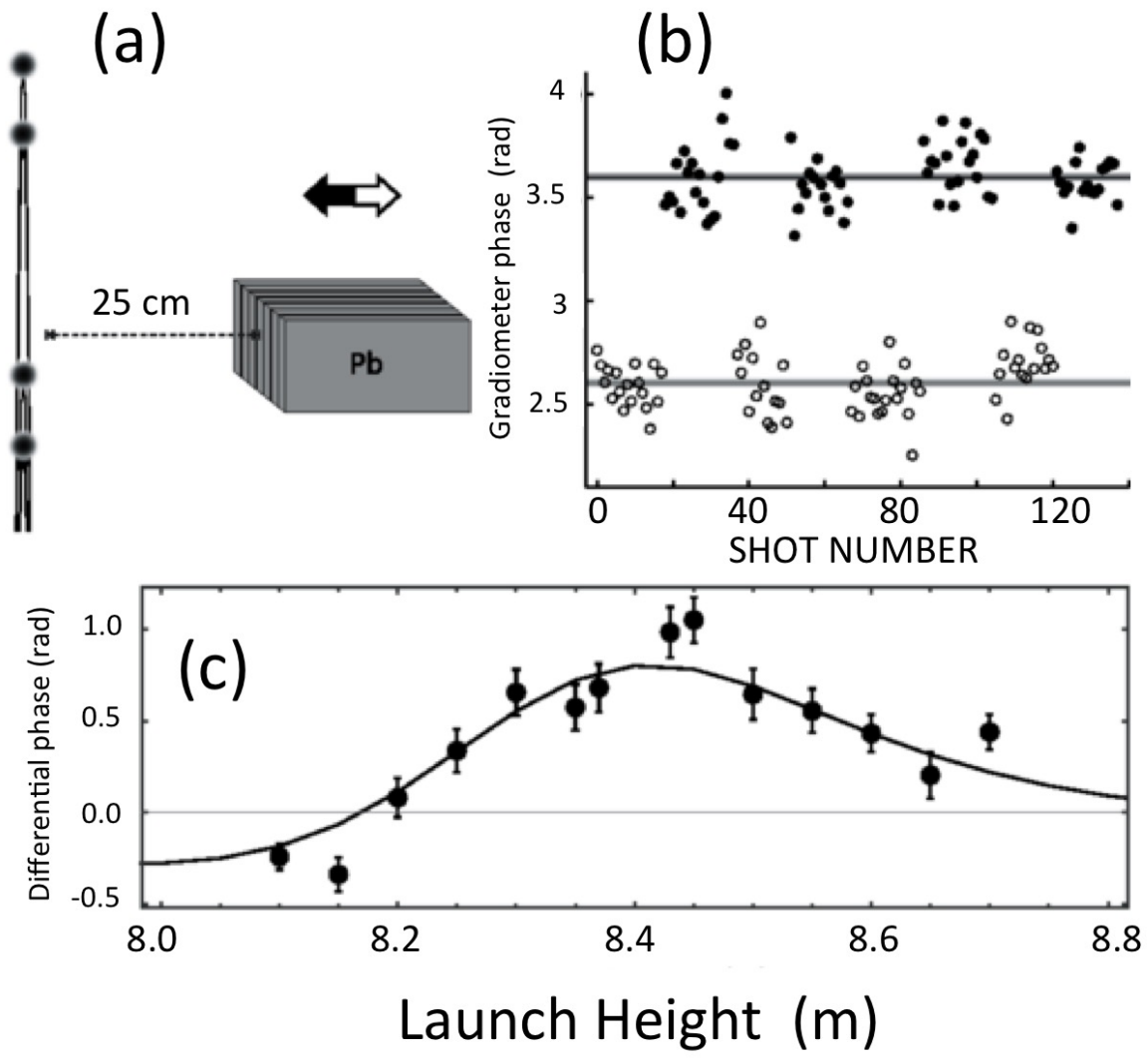

Figure 10: (a) Schematic of the experimental setup for measuring the gravity gradient produced by seven lead bricks. (b) Measured gradiometer phases for a sequence with (solid circles) and without (open circles) the bricks present. (c) Gradiometer phase difference (with and without bricks present) as a function of the launch height. The black, solid curve represents the full phase shift calculation. 
macroscopic states were generated using large momentum transfer (LMT) atom optics and long drift times. This allowed the first observation of the influence of space-time curvature across a single quantum system.

- Measuring the Earth's rotation with an atom interferometer. High sensitivity dual-axis gyroscope [60] and precision gyrocompassing [61] measurements make use of spatially resolved atom detection with CCD cameras and long drift times. A mirror on a piezo tip-tilt stage is used to counter-rotate the laser beams against the Earth's rotation and compensate for Coriolis forces. Gyrocompassing allowed the Earth's rotation-axis to be determined, and the correct rotation rate $(57.9 \mu \mathrm{rad} / \mathrm{s}$ at Stanford $)$ to be measured with a precision of $200 \mathrm{nrad} / \mathrm{s}$.

- Suppression of gravity gradient systematic errors in a dual species interferometer [12]. Searches for new forces and tests of the Principle of Equivalence can be made by comparing interferometric measurements of different atomic species. However, gravity gradients couple to initial kinematic offsets between atomic species, which leads to systematic errors. A method to suppress these systematic errors by shifting the laser frequency for the mirror pulses has been demonstrated. The correct value of the frequency shift depends on a weighted time-average of the gravity gradient the atoms experience. The empirically determined value agrees well with the value calculated from gravity gradient measurements as a function of height [1]. The technique enabled a precision of $\Delta \mathrm{g} / \mathrm{g} \sim 6 \times 10^{-11}$ per shot to be achieved.

- Demonstration of matter wave lensing protocols to produce atom clouds with effective temperatures down to $50 \mathrm{pK}$ in the transverse dimensions [53]. The observation of such low temperatures enables limits to be set on proposed modifications to quantum mechanics in the macroscopic regime.

Experience from the present generation experiment has enabled the sources of systematic uncertainty that are relevant to MAGIS-100 to be identified. These are listed in the appendices $(\mathrm{H}$ and I) which also describe the strategies that can be used to mitigate and/or measure their effects.

\section{Technological advances required for MAGIS-100}

A steady stream of technical advances have enabled the $100 \mathrm{~m}$ scale experiment at Stanford to be successful. Those advances continue, and MAGIS-100 will provide both a motivation and mechanism for continued technical progress, and an opportunity to integrate the various advanced technologies into an experiment. Some aspects of the development needed for MAGIS-100 involve a direct scaling of methods already developed for the Stanford $10 \mathrm{~m}$ atomic fountain and hold a relatively low amount of technological risk. These components of MAGIS-100 are: launching of the atom clouds [60], attaining sufficiently low atom cloud temperature [53], atom source integration into the larger apparatus [60], increased laser power [62], and accommodating larger Doppler shifts of the atomic transitions during the interferometer and during the atom detection process $[1,12,17,60,61]$. Other aspects of MAGIS-100 require a more significant adaptation of existing methods or the replacement of these methods with appropriate alternatives. This progress is also needed to prepare the way for the km-scale experiment, and includes: mitigating/characterizing laser wavefront aberrations, compensating for Coriolis forces on the atoms from Earth's rotation, laser beam alignment, atom trajectory control, magnetic shielding and magnetic field control, and maintaining the necessary vacuum quality. These aspects are further discussed in Section 6 and Appendix $\mathrm{H}$. 


\section{Requirements for a $100 \mathrm{~m}$-scale experiment}

Based on the experience gained with the construction and operation of the successful $10 \mathrm{~m}$-scale experiment, the following summarizes the requirements that must be met for a $100 \mathrm{~m}$-scale experiment. The technical improvements needed for MAGIS-100 are also discussed.

\subsection{Site}

The site must provide a long vertical shaft in which the gradiometer will be installed.

- The length of the shaft (plus overhead covered space) must be close to $100 \mathrm{~m}$ or greater.

- The diameter of the shaft must be sufficient to enable installation of the setup.

- There must be crane coverage over the shaft, also to enable installation.

- There must be sufficient space at the top and through the shaft for the laser system.

- The vibration environment must be well characterized to enable an effective design of the experiment. Vibrations can affect the interferometer in several ways. Mechanical vibrations (typically in the range $10 \mathrm{~Hz}-1000 \mathrm{~Hz}$ ) can cause misalignment of the laser system. In addition, lower frequency vibrations, which cause motions on time scales comparable to the free-fall time (in the range of $1 \mathrm{~Hz}$ and below) will contribute to the systematic uncertainty of the measurement by coupling to spatial phase variations in the laser lightfront. Once the vibrations in the environment have been adequately characterized, their impact can be mitigated by careful location of sensitive components and any necessary vibration isolating mounting.

- The magnetic environment must be characterized. MAGIS-100 will use strontium atoms which have reduced sensitivity to magnetic fields compared to the alkali atomic species typically used in interferometry. However, the magnetic fields in the experimental area still need to be accounted for, and compensated where necessary. In particular, a time varying magnetic field in the frequency band of the detector can fake a signal, and must be sufficiently suppressed. Magnetic field measurements, including temporal field characterization and monitoring, are needed at several points in the shaft to determine the level of passive and active shielding that the detector will require.

- Temperature variations must be characterized. Temperature fluctuations affect the energy and temperature stability of the lasers, and also affect the alignment of optical components. In addition, the temperature of the atom source must be controlled to $1-2{ }^{\circ} \mathrm{C}$.

- The site must be able to provide a laboratory environment with the required power to operate the experiment, appropriate support for laser and vacuum systems, and safety oversight.

\subsection{Vacuum and Vacuum pipe}

Compared with the Stanford 10m scale experiment, MAGIS-100 will have longer atom drift times and will require a better vacuum pressure to reduce atom loss due to collisions with residual gases. In the Stanford $10 \mathrm{~m}$ fountain, vacuum of sufficient quality is achieved with a pair of high-volume pumps on either end of the fountain. MAGIS-100 will have pumps at periodic intervals along the vacuum pipe. The vacuum and vacuum pipe have the following features: 
- The vacuum pressure is in the UHV range to support long lifetimes for the atomic clouds. For optimal performance, the vacuum pressure must be on the order of $10^{-11}$ Torr at the pumps, and $10^{-10}$ Torr is the maximum pressure allowed between pumps.

- The vacuum pipe diameter is expected to be between $10 \mathrm{~cm}$ and $20 \mathrm{~cm}$. The pipe will be made of stainless steel or aluminum with vacuum connections that use standard ConFlat flanges. All-metal gate valves will separate the sources from the main pipe to allow efficient reconfiguration and maintenance. Near the vacuum pumps, there will be additional ports to install ion gauges, roughing and leak-checking ports, and viewport windows. All materials contained within the vacuum system must be suitable for UHV with respect to cleaning, hydrocarbon contamination, and out-gassing rates, and must withstand a minimum of $100{ }^{\circ} \mathrm{C}$ bake temperature.

- The pipe has a series of connection nodes along its length to interface with the atom sources. The nodes are fitted with viewports that are used to implement the atom shuttle and launch with appropriate laser beams, and to provide optical access for imaging.

- At the top of the pipe a transition section interfaces with the atom optics laser system. The pipe contains an in-vacuum telescope and a mode filtering region for delivering laser pulses with minimum aberration.

\subsection{Magnetic shielding and magnetic field control}

A horizontal magnetic field ("bias field") is applied to pin the atomic spin in a well-defined direction (i.e., "define the quantization axis"). This can be produced using vertical coils outside of the vacuum pipe. Spatially and temporally varying stray fields, which can induce spurious phase shifts, are minimized using a magnetic shield around the interferometer region. For a $100 \mathrm{~m}$ interferometer height, it will be necessary for the shield to be composed of multiple vertical segments. Compensation coils can be used to appropriately trim residual fields at the gaps between segments. The MAGIS-100 shield design will require some finite element modeling. The design of such systems is well-understood [63].

As previously discussed in Section 3.3, the magnetic field sensitivity of the Sr atoms that MAGIS-100 will use is several orders of magnitude less than the corresponding sensitivity for the alkali atoms typically used in previous atomic fountains. For the fermionic ${ }^{87} \mathrm{Sr}$ isotope, a comagnetometer configuration using atoms in states with both positive and negative Zeeman shifts can eliminate the linear phase shift sensitivity to magnetic fields [64]. Moreover, the AC nature of a gravitational wave or dark matter signal suppresses the influence of DC phase shift offsets from temporally static magnetic field gradients. If needed, temporal variations in the magnetic field can be locally measured and compensated.

\subsection{Atom source}

A commercial atom source ${ }^{4}$ can deliver a high-flux beam of atoms that can be cooled to nanokelvin temperatures and subsequently used for interferometry. The atom source itself consists of a vacuum chamber that interfaces with the interferometer region, an optical breadboard for the lasers needed to implement the cooling, and associated control electronics for the lasers. The atom source also includes a number of magnetic coils necessary for atom cooling and their associated current

\footnotetext{
${ }^{4}$ For example, the cold atomic beam sources for alkaline-earth precision experiments from AOSense, inc.
} 
supplies. The atoms are collected in a magneto-optical trap (MOT). The MOT consists of twostages which are distinguished by the different wavelengths of laser light needed: the blue MOT is for initial atom collection followed by the red MOT for additional cooling. To reach the necessary atom temperatures, a high-power dipole trap laser is used to produce a conservative potential to implement evaporative cooling and delta-kick cooling. The laser light is sent into the cooling chambers through vacuum viewports. All the lasers for the MOTs and for the commercial atom beam source are housed inside the atom source enclosure. The approximate size of the source is $60 \times 100 \times 70 \mathrm{~cm}^{3}$. The enclosure protects the lasers from the elements and maintains a stable temperature to prevent misalignment of the optics.

Given the long MAGIS-100 free fall durations compared with the $10 \mathrm{~m}$ scale experiment:

- The atom ensembles must be colder to prevent them from expanding too much with respect to the transverse size of the atom optics laser beam. Substantial evaporative cooling and advanced atom lensing techniques are necessary [53]. Lensing protocols for MAGIS-100 will be based on those already demonstrated in the Stanford $10 \mathrm{~m}$ fountain, which are able to provide sufficiently low temperature (as low as $50 \mathrm{pK}$ ) [53].

- Tighter control over the initial velocities of the atom clouds is needed in order to reduce unwanted phase shifts from velocity dependent forces (e.g., from rotations or gravity gradients) [58] or from the coupling of imaging distortions to the final atom cloud position. Velocity jitter can be dramatically reduced, for instance, with atom lensing sequences making use of optimal control theory [65]. Moreover, spatially resolved detection can be used to characterize initial cloud kinematics for each experimental shot and correct for corresponding phase shifts in post-processing. The long drift times available in MAGIS-100, which correspond to a highresolution in velocity measurements, will enable evaluation of improved lensing sequences and of post-processing correction of velocity-dependent phase shifts.

The need to integrate the atom source with the interferometer constrains the source design. For instance, the Stanford $10 \mathrm{~m}$ fountain requires large diameter atom optics laser beams to pass through the atom source chamber, putting a lower bound on the chamber's size. Similar constraints must be accounted for in the MAGIS-100 design. In MAGIS-100, it appears ideal to have atom sources in independent vacuum chambers attached to the side of the main, $100 \mathrm{~m}$ tall chamber. Atom cloud preparation can then proceed simultaneously with the interferometry, improving the instrument bandwidth. This setup requires horizontal lattice shuttling or launching to transfer atoms from the source chambers to the main chamber. The lattice launching schemes demonstrated in the Stanford $10 \mathrm{~m}$ fountain can be adapted for this purpose.

The atom source must produce atom clouds at a sufficient rate so that the interferometer phase measurements can be made at the Nyquist rate for the signal in question. For many planned measurements, this requires that new atom clouds are available before interferometry is finished with the previous cloud. Stray light is shielded with appropriate baffles and apertures to allow for near continuous atom cooling that overlaps with the interferometer measurements. We initially require that each atom source provides $10^{6}$ atoms/s. The MAGIS-100 program will include R\&D aimed at increasing this rate by up to two orders of magnitude.

\subsection{Transfer and Launch}

The cooled atom ensembles are transported from the atom source chamber to the 100 meter interferometer region using a combination of optical dipole trapping lasers and an optical lattice shuttle. Optical forces launch the atoms at the beginning of the interferometer sequence. For a taller interferometer height, atoms launched upwards need to receive a larger velocity kick, implying the need 
for more efficient momentum transfer. At the same time, launch-induced heating of the atoms needs to be reduced. Meeting these constraints requires increased laser power and greater laser frequency agility. The Stanford $10 \mathrm{~m}$ fountain launches the atoms via coherent acceleration in an optical lattice potential [60]. This method yields highly efficient momentum transfer and a dramatically reduced heating rate. The predominant limitation of this method arises from heating of the atoms due to residual spontaneous emission. For a fixed optical lattice depth, the spontaneous emission rate can be linearly reduced by linearly increasing both the laser power and detuning [66]. For MAGIS-100, the amount of momentum transferred by the lattice must increase by $\approx 3$ over that transferred in the Stanford $10 \mathrm{~m}$ fountain, implying a reduction in the spontaneous emission rate by the same factor. Commercially available lasers of sufficient power can meet this requirement. The increased laser frequency agility needed to accommodate the larger Doppler shifts associated with the larger atom velocities will be implemented by acousto-optic or electro-optic frequency shifting.

\subsection{Atom optics laser system}

The interferometer laser system is located at the top of the shaft and the light pulses are delivered though a telescope and mode filter mentioned above. The laser system has the following characteristics:

- The laser is on an optical breadboard that is vibration isolated and is protected from the environment by a temperature-stabilized enclosure.

- The laser is delivered to the top of the 100 meter inteferometer region through a vacuum tube with an in-vacuum beam shaping telescope and turning mirrors. This ensures that the beam has adequate distance to propagate in vacuum with minimal added aberration, acting like a spatial filter to provide a uniform laser wavefront at the location of the atoms.

- The laser operates on the Sr clock transition $(698 \mathrm{~nm})$.

- The diameter of the laser beam is large (several centimeters) to minimize diffraction and to provide a uniform intensity and wavefront across the (expanding) atom clouds. As a result, the required laser power is large $(4 \mathrm{~W})$. The laser system will be based on coherently combined commercially available Ti:Sapphire lasers.

- For a larger interferometer height or longer baseline, the effect of any laser beam angular misalignment is magnified. Therefore, improved angular stability must be realized. Appropriate care needs to be taken in designing the optomechanical setup and in controlling the ambient temperature. The use of monolithic, steel optomechanics can substantially reduce alignment drifts. Moreover, split quadrant detectors can provide real-time alignment characterization, which could be used in combination with motorized or piezo-actuated mirror mounts to provide active alignment stabilization if necessary.

- The laser is frequency stabilized to an optical cavity to reduce its linewidth. The laser frequency must be stable to allow for efficient light pulses. The stability requirements are therefore determined by the required total number of pulses for an interferometer sequence. We require the $698 \mathrm{~nm}$ laser to be stabilized to $10 \mathrm{~Hz}$.

- The amplitude and frequency of the laser are controlled using acousto-optic modulators (AOMs). The amplitude control is used to form the intensity profiles of each pulse as a function of time, and the frequency control is used to shift the light onto resonance with the (Doppler-shifted) atom clouds. Note that atoms in a taller atomic fountain will attain 
a larger free-fall velocity and therefore have larger Doppler shifts with respect to the atom optics laser beam. This places more demanding requirements on the laser frequency range and agility. Acousto-optic frequency shifting or agile offset locking to a reference laser can be used to meet this challenge. By using multiple modulation frequencies, the atom optics laser can emit more than one frequency at a time. This is needed when there are multiple atom clouds with different Doppler shifts that must receive light pulses at the same time, as may arise when implementing Doppler-based multiplexing schemes.

- To implement LMT pulse sequences, sequential laser pulses are applied from alternating directions. Although the atom optics laser is delivered only from the top, pulses traveling from the bottom to the top can also be realized because of the retro reflection mirror at the bottom of the tube. This mirror ensures that all laser pulses propagate in both directions. Whether an atom absorbs an upward or downward traveling pulse is determined by setting the frequency of the pulse, since the two cases have opposite Doppler shifts.

- In addition to driving single-photon transitions on the $698 \mathrm{~nm}$ clock transition, the same laser system can be easily reconfigured to implement two-photon Bragg transitions. For a single atom source, Bragg transitions can be used effectively for overlapped dual-species experiments (such as searches for time-varying new forces). Bragg atom optics may be preferred when comparing two isotopes of $\mathrm{Sr}$, as the clock transition is naturally coupled only in ${ }^{87} \mathrm{Sr}$, but Bragg transitions near the $461 \mathrm{~nm}$ line are possible for bosonic isotopes as well.

\subsection{Laser wavefront aberrations}

Aberrations in the atom optics laser beam result in laser phase shifts that depend on the transverse position of the atom cloud with respect to the aberrations. Since the atom ensembles are at different heights, aberrations can vary from one atom ensemble to the other due to diffraction, leading to unwanted differential phase shifts that can be a source of noise if either the initial atom kinematics (position, velocity, and temperature) or the wavefront aberrations vary in time. These effects have been extensively analyzed [22]. For a longer baseline, diffraction effects are increased. Moreover, the larger amount of momentum transfer envisioned for MAGIS-100 increases the interferometer

phase response to wavefront aberrations. Therefore, improved laser beam quality and better in situ wavefront diagnostics are needed.

For the $10 \mathrm{~m}$ scale experiment, spatially resolved detection of the atom interferometer phase at different points within the atom cloud has proven essential for mitigating phase errors from wavefront imperfections, allowing the atoms to provide in situ information about wavefront perturbations [60]. This method, in combination with point source interferometry [60] and the interleaving of shorter duration interferometers optimized to be maximally sensitive to local wavefronts [22], can be used for in situ wavefront characterization in MAGIS-100. Temporal variations in the wavefront can then be measured, and phase shifts arising from the coupling of initial atom kinematics to wavefront aberrations can be determined and corrected for by measuring the initial cloud position, velocity and temperature for each experimental shot-once again making use of spatially resolved detection $[1,12,17,60,61]$ to do so. One can discriminate between initial position and initial velocity fluctuations by making an independent position measurement prior to the start of the interferometer. This independent position measurement could be done, for example, by taking an absorption image of the cloud that only destructively measures a small fraction of the atoms.

Improved laser beam quality will be realized via high-quality delivery optics and spatial filtering of high transverse spatial frequency ( $\lesssim 1 \mathrm{~mm}$ spatial scale) perturbations by free propagation of 
the beam by $\sim 10 \mathrm{~m}$ before interacting with the atoms. Free-propagation spatial filtering has been successfully implemented for optical dipole lensing of the atoms in the Stanford $10 \mathrm{~m}$ fountain [53].

\subsection{Tip-tilt mirrors and rotation compensation}

At the bottom of the interferometer pipe is a mirror. The mirror is actuated using an in-vacuum tiptilt stage to rotate the atom optics and hence laser beam. A rotation of $\mu \mathrm{rad} / \mathrm{s}$ is used to mitigate the effect of the rotation of the Earth which leads to Coriolis forces that cause velocity-dependent interferometer phase shifts. When the interferometer height-and correspondingly its sensitivity-is made larger, these phase shifts become increasingly problematic. The effect of Coriolis forces can be compensated by using the mirror to counter-rotate the atom optics laser beam against the rotation of the Earth, as demonstrated in the Stanford $10 \mathrm{~m}$ fountain and other apparatus [60,67]. The correct rotation angle and rate can be determined to the necessary accuracy following the method demonstrated in [61]. A similar setup with a mirror on a piezo tip-tilt stage will also be used at the top of the apparatus for directing the laser beam down into the $100 \mathrm{~m}$ pipe.

For $100 \mathrm{~m}$ or longer interferometer heights and/or baselines, substantial deflections of the rotation-compensated laser beam with respect the atom ensembles will become an important new challenge. A strategy to address this challenge is to use local optical lattices to independently launch vertically separated atom ensembles with different horizontal velocities so that all atom ensembles remain near the center of the deflected atom optics laser beam. Alternatively, multi-loop interferometers with highly suppressed sensitivity to rotations can be used in order to alleviate the need for rotation compensation-and the corresponding beam deflection-altogether [22,68]. Rotation compensation can also be used in conjunction with multi-loop interferometry.

The benefits of having mirrors on piezo tip-tilt stages with controllable angles extend beyond the ability to implement rotation compensation. The ability to apply different mirror tilts for different interferometer pulses allows an interferometer phase shift to be controllably imprinted that is equal to a tunable scale factor multiplied by an arbitrary, tunable linear combination of the initial atom position and velocity and the transverse dimensions. This linear combination is determined by how much the mirror is tilted for each interferometer pulse. For example, if the mirror is tilted by an angle $\theta_{\mathrm{m}, i}$ in the $x$ direction for the $i$ th interferometer beam splitter or mirror, an interferometer phase that scales as $n k \theta_{\mathrm{m}, i}\left(x_{0}+v_{x 0} t_{i}\right)$, where $n$ is the number of photon momentum kicks, $k$ is the laser wave number, $x_{0}$ is the initial position along the $x$ axis, $v_{x 0}$ is the initial velocity along the $x$ axis, and $t_{i}$ is the time at which interaction occurs [61]. An analogous expression applies for the $y$ axis, or for a tilt along an intermediate axis in the $x y$ plane. A phase shift that is proportional to an arbitrary linear combination of $x_{0}$ and $v_{x 0}$ can be achieved by choosing different values of $\theta_{\mathrm{m}, i}$ for at least two different values of $i$ (this has already been demonstrated at Stanford, not yet published). For example, a tilt of the mirror angle for the final beam splitter produces an interferometer phase that is proportional to $x_{0}+v_{x, 0} t_{f}$, where $t_{f}$ is the time of the final beam splitter. This leads to horizontal spatial fringes across the interferometer output ports (phase shear readout), which are useful for phase readout purposes $[1,12,61]$. More generally, the ability to generate a phase shift of the form $c_{x 0} x_{0}+c_{v x 0} v_{x 0}$ for adjustable coefficients $c_{x 0}$ and $c_{v x 0}$ is a valuable tool to compensate for interferometer phase noise arising from the coupling of initial kinematics to external perturbations such as rotations, gravity gradients, or wavefront perturbations (see Sec. 6.4 and Sec. 6.7) ${ }^{5}$. To leading order, let us say that the interferometer phase associated with the coupling of initial kinematics to all external perturbations has the form $a_{x 0} x_{0}+a_{v x 0} v_{x, 0}$, for constants $a_{x 0}$ and $a_{v x 0}$. These constants can be empirically measured by varying $x_{0}$ and $v_{x 0}$, and by tuning of the angles

\footnotetext{
${ }^{5}$ As above, the $x$ axis is taken as an example, and all expressions can be generalized to any axis in the $x y$ plane, with $z$ defined as the vertical axis.
} 
$\theta_{\mathrm{m}, i}$, we can set $c_{x 0}=-a_{x 0}$ and $c_{v x 0}=-a_{v x 0}$. The linear phase response to initial kinematics is thereby cancelled ${ }^{6}$. The rotation compensation method described above is one example of this more general method. Also, the more general method described here is similar in spirit to the gravity gradient compensation technique demonstrated in [12]. If multi-loop interferometers are used to suppress leading order rotational couplings, the required tilt angles can be sufficiently reduced so that large beam deflections are not a concern.

\subsection{Controls and monitoring}

The real-time control of the interferometer will be based on an FPGA based sequencer, controlling multiple devices via differential and TTL pulses, including Acousto-Optical and Electro-Optical Modulators (AOMs \& EOMs), optical shutters, electro-magnet power supplies, cameras and photodiodes. Attached devices, for example the optical shutters and electromagnets, will require additional circuitry to control their function. The lasers for the MOT will be frequency-stabilized using spectroscopic techniques (in combination with linewidth narrowing via locking to an optical cavity for the red cooling laser). The frequency stabilization can be performed with off-the-shelf low-level $\mathrm{RF}$ components and feedback electronics.

Monitoring will utilize Ethernet, GPIB, and USB interfaces to power supplies and environmental monitors. Laser light levels monitored by photo-diodes will be read out via ADCs synchronized with the FPGA boards and oscilloscopes where necessary.

\subsection{Cameras and Data Acquisition}

To detect and measure the atom ensembles, scattered near-resonant photons first decelerate the atoms to rest. The atoms then continue to resonantly scatter light, which is imaged onto a highresolution CCD camera. The interferometer phase is extracted by analyzing a spatial fringe pattern across the ensemble $[1,12,61]$. As observed in the Stanford $10 \mathrm{~m}$ fountain, heating and distortion of the atom cloud during the deceleration process can affect the imaged atom fringe pattern and introduce apparent phases shifts. An optimized laser frequency ramp during deceleration is used to mitigate these effects in the Stanford $10 \mathrm{~m}$ fountain. This method can be directly scaled to accommodate the $\approx 3$ times larger velocities in MAGIS- 100 .

Due to the low cycling rate $(1-2 \mathrm{~Hz})$ of the interferometer, there is ample time between measurements to fully readout imaging from the various cameras and to digitize signals using ADCs. The task for the data acquisition is to collate this data (the raw data comprises optical CCD images of the resonantly scattered light from the atoms in the two different states), with data from the monitoring system, so that data quality may be assured.

Multiple cameras from different angles will be used in each detection region within the main vertical pipe. Following the work in the Stanford $10 \mathrm{~m}$ tower $[1,12,61]$, two cameras that view the cloud from the side at orthogonal angles will be used to detect horizontal spatial fringes across the cloud and to measure the position and size of the detected cloud in all three dimensions for each

\footnotetext{
${ }^{6}$ For this method to be helpful in a gradiometer setup, the coefficients $a_{x 0}$ and $a_{v x 0}$ need to be nearly the same for the different interferometers. This will tend to be true for the coupling of initial kinematics to rotations or gravity gradients. However, owing to diffraction, this will not automatically be the case for wavefront perturbations. To make these coefficients the same between different interferometers for the wavefront perturbation case, one possibility is to independently adjust the initial cloud positions in the $x y$ plane for the different interferometers so that similar coupling coefficients are reached. Alternatively, the wavefront can be engineered (for example, via a deformable mirror or spatial light modulator) in order to differentially tune the coupling coefficients between different interferometers so that these coefficients reach similar values. These methods will be explored as part of the research and development method described in Sec. 8 to improve interferometer performance.
} 
experimental shot. These position and size measurements after the interferometer is completed, in combination with an initial position measurement before the interferometer is started (see Sec. 6.7), allow for corrections in post-processing of noise induced by the coupling of cloud kinematic jitter to rotations, wavefront aberrations, and spatial variations in external fields, as discussed in Sec. 6.4 and in Appendix H. Additionally, the system will have the capability to use the same shuttle that transfers the atoms from the atom source chamber to the interferometry region to transfer atoms back into the atom source chamber for detection after the interferometer sequence is completed. A camera below the atom source chamber will then be able to detect the interferometer output ports from a bottom view, which is valuable for in situ measurements of the optical wavefront using the atoms (see Sec. 6.7). Shuttling the atoms back into the atom source chamber may be used for collecting diagnostic data for wavefront characterization. Most other data will utilize detection in the main vertical pipe, as described above.

The experiment will use the same family of back-illuminated, commercially available cameras as that implemented in the Stanford $10 \mathrm{~m}$ fountain [12]. These cameras have a quantum efficiency of approximately $90 \%$ and a CCD size of $1340 \times 400$ pixels, with each pixel having a $20 \mu \mathrm{m} \times 20 \mu \mathrm{m}$ size and a 3 electron rms read noise. The camera allows user-defined groups of pixels to be binned together in hardware to reduce the influence of electronic read noise.

\subsection{Computing}

The computing and networking needs are modest. The requirements for the interferometer are a multi-core control machine to take data from the interferometer and monitoring systems, as well as a SOIC-based computer for ease of communication to the FPGAs. An additional machine will be required to analyze the images from cameras in the readout and monitoring systems and to monitor the status of the experiment.

Raw-data will take the form of rasterized images, we predict that this will be approximately 1-2 TB of data daily, which can be highly compressed (initial tests suggest a factor of at least 30 is easily achievable). The data must be stored in an archive, for example in the Fermilab tape archive. The networking needs are such that they can be serviced by the infrastructure already present for the existing neutrino experiments.

\section{MAGIS-100 at Fermilab}

The existing NuMI access shaft (Fig. 11) is $88 \mathrm{~m}$ deep (measured from the building floor to the roof of the underground tunnel), and is covered by the MINOS Service Building, which should permit a total drop distance exceeding $90 \mathrm{~m}$. This offers a compelling opportunity to realize MAGIS100. The top of the shaft is shown in Fig. 12. In addition to providing access for installation and maintenance of the experiment, there is also space for installation of laser equipment. The site meets all of the requirements listed in Section 6.1. The site is currently being measured to characterize the environment (vibrations, temperatures, magnetic fields) which will facilitate the detailed design of the experiment.

\subsection{Layout}

A CAD rendering of the detector in the NuMI shaft is shown in Fig. 13. The primary components of the system are the $\sim 90 \mathrm{~m}$ vertical vacuum $\mathrm{pipe}^{7}$ and three atom sources located at the top,

\footnotetext{
${ }^{7}$ The pipe will extend a few meters beyond the top of the $88 \mathrm{~m}$ deep shaft
} 

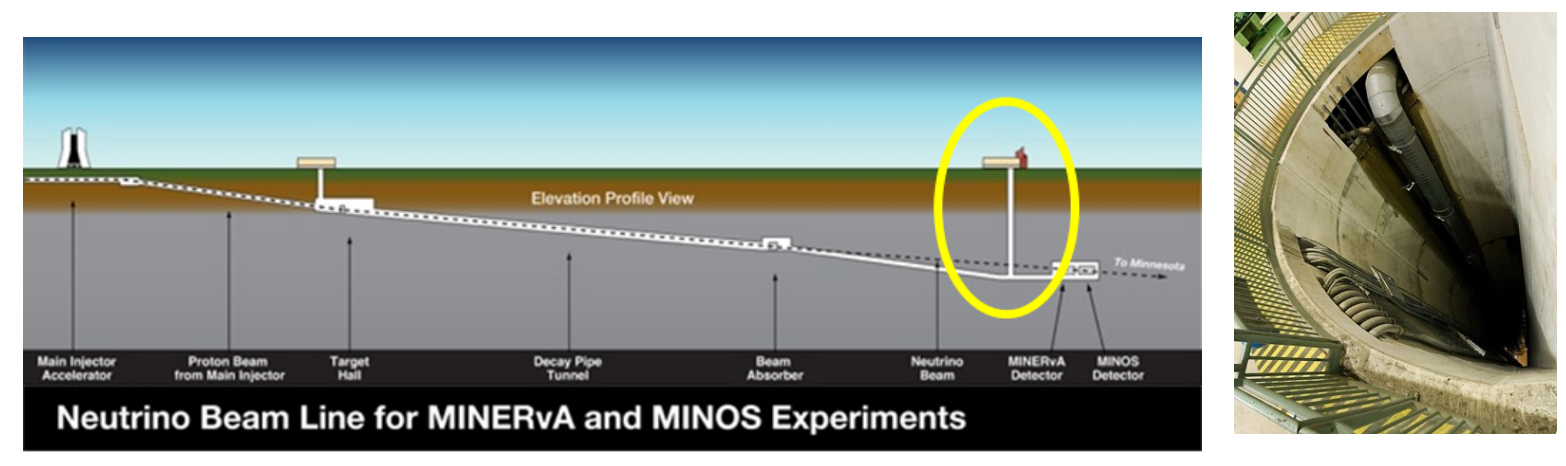

Figure 11: Proposed site for MAGIS-100 (left figure). Elevation profile view of existing NuMI (Neutrino Main Injector) tunnel at Fermilab. MAGIS-100 will be located in the $100 \mathrm{~m}$ deep access shaft (yellow circle). The detector is to be mounted along the wall of the $88 \mathrm{~m} \mathrm{NuMI}$ access shaft (right figure).

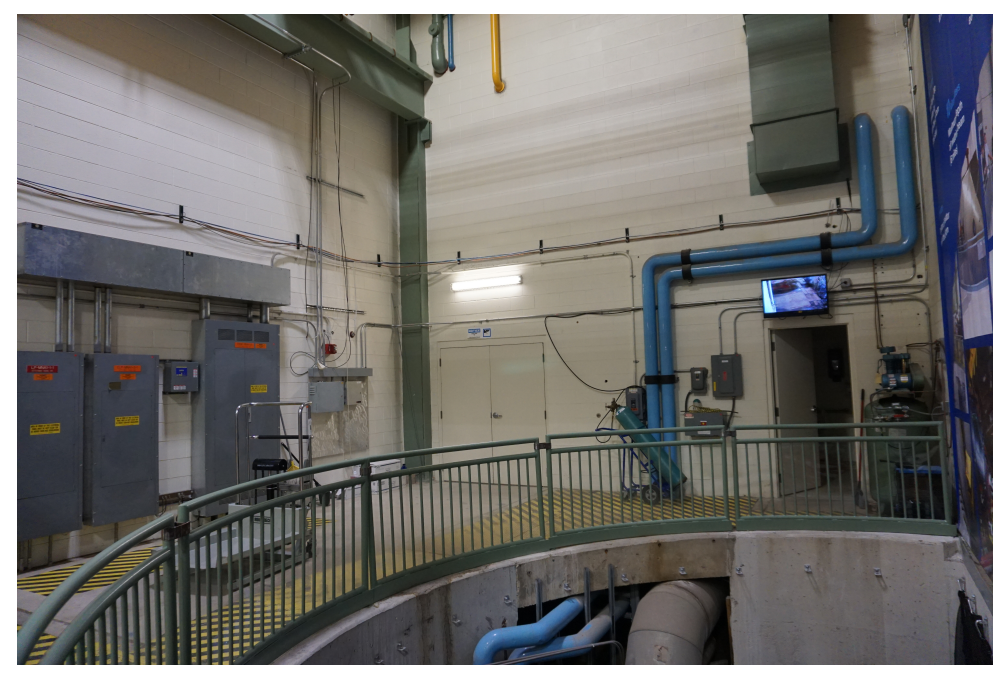

Figure 12: View of top of shaft and possible spaces for installation of laser equipment 


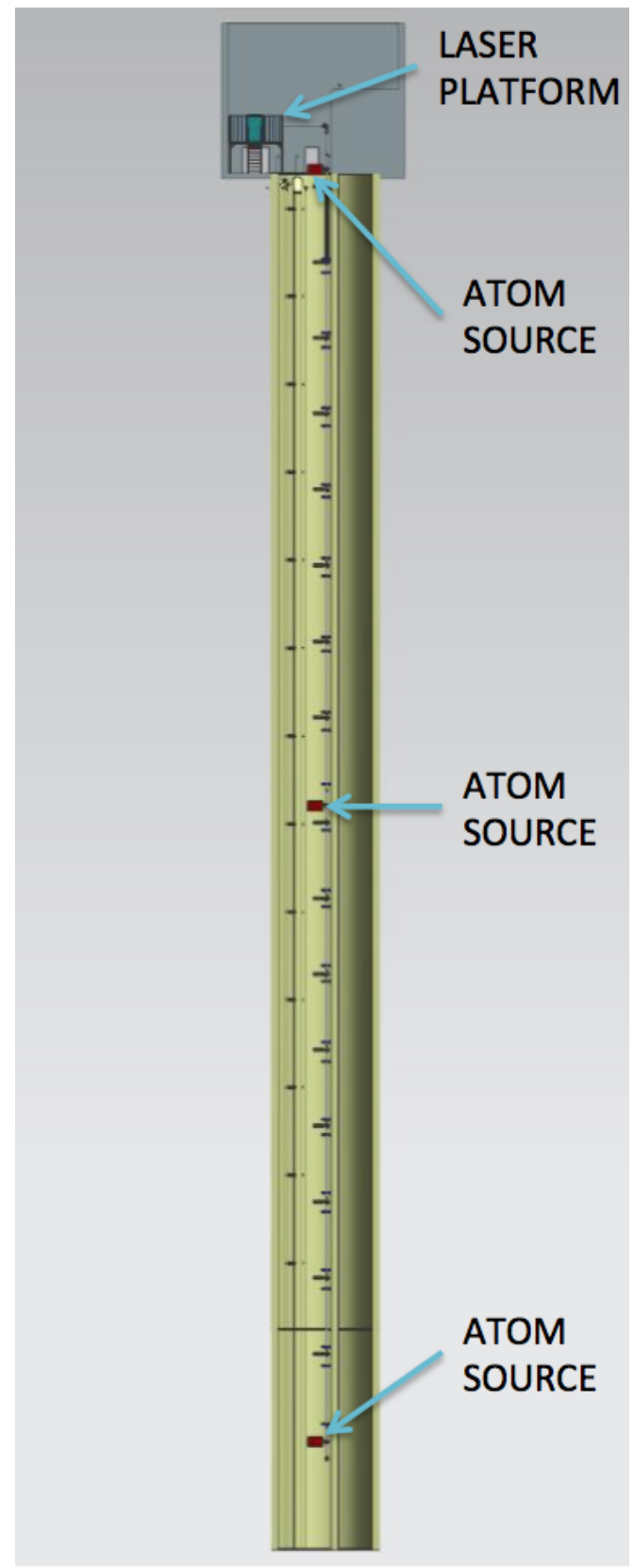

(a) Detector side view.

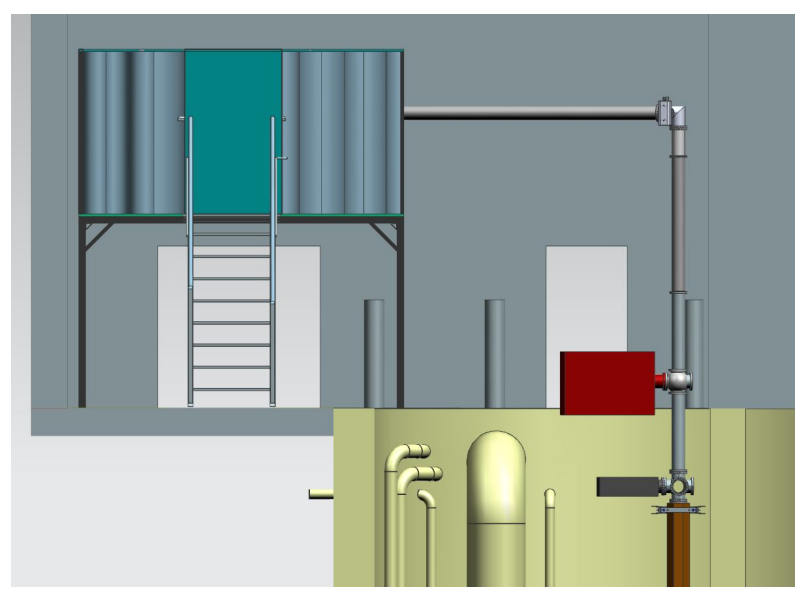

(b) Top

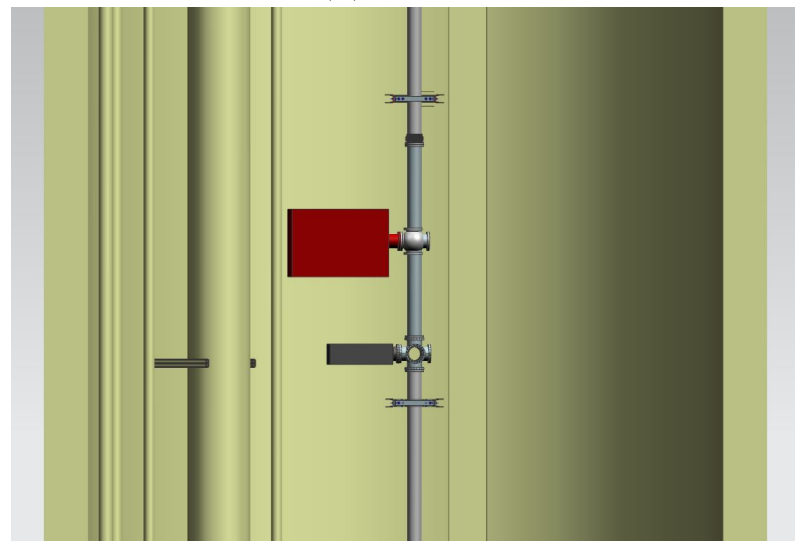

(c) Center

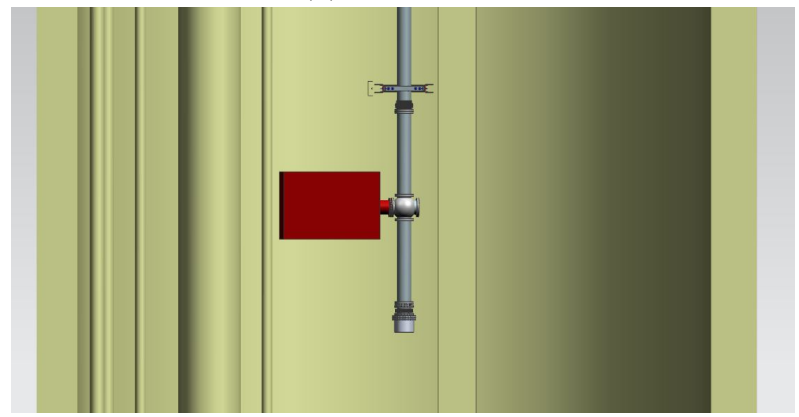

(d) Bottom

Figure 13: MAGIS-100 detector conceptual CAD model. The side view (a) shows a cross section of the existing $\sim 90 \mathrm{~m}$ underground NuMI shaft, with the MAGIS-100 vacuum tube installed. The three atom sources are attached at the (b) top, (c) middle, and (d) bottom of the detector. A laser hutch at the top of the shaft contains the interferometry lasers. The laser light enters the vacuum system at the top of the shaft through a vacuum viewport and then propagates downwards inside the vacuum tube. 


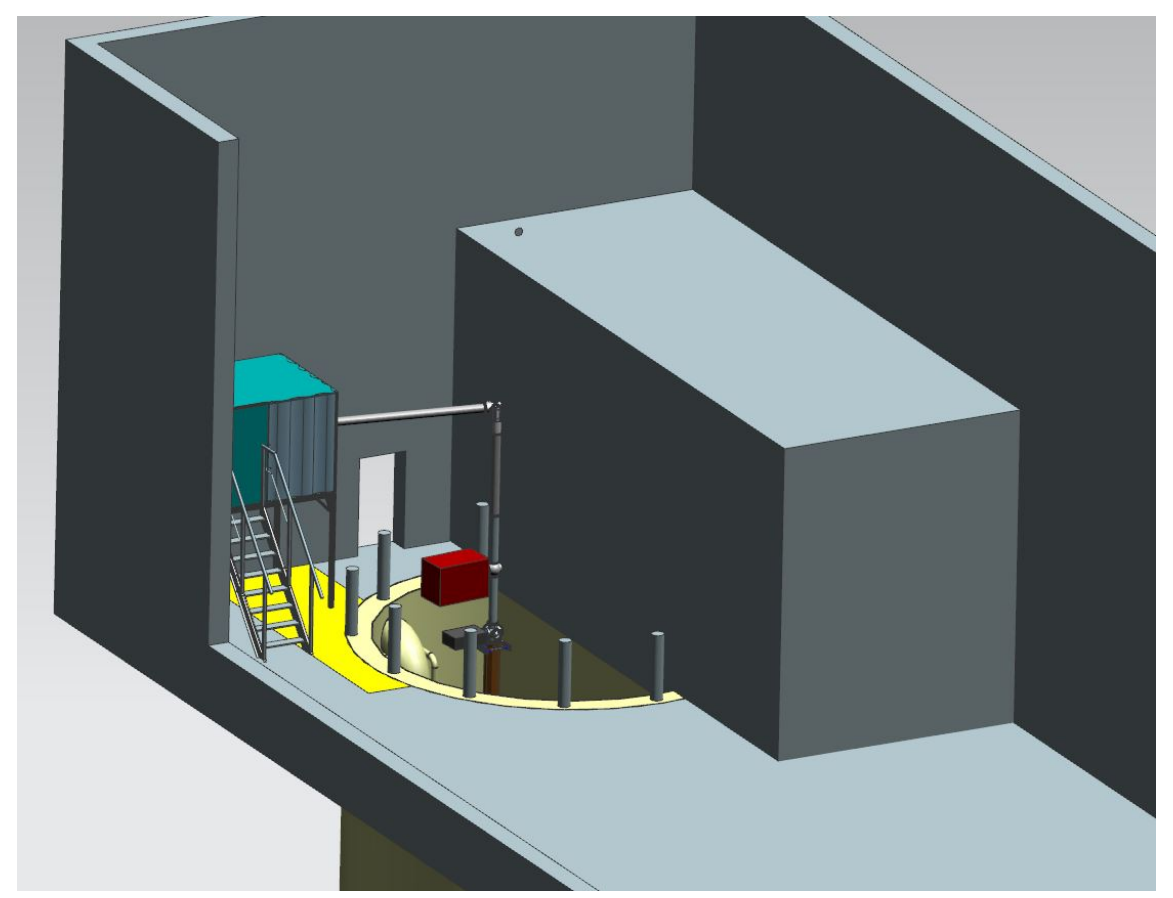

Figure 14: The layout of the top of the detector showing the laser hutch placement on a dedicated platform (green) and the atom source placement (red).

middle, and bottom of this pipe. The layout of the top of the shaft (and laser hutch placement) is shown in Fig. 14, a plan view locating the vacuum pipe within the shaft is shown in Fig. 15, a CAD rendering of one of the atom sources is shown in Fig. 16, and the atom source optics layout is shown in Fig. 17. Note that:

- The physical layout of the equipment will fit well within the available space in the MINOS building and shaft.

- The MINOS Service Building is equipped with a 15 ton bridge crane. The crane has a hard stop with a clearance from the west wall of approximately 4'8", which positions the hook such that the west side of the shaft can be accessed by an existing or upgraded personnel basket. Installation of equipment will require additional temporary lifting equipment such as a mobile gantry.

- Lasers, controls, and additional support systems will be located at the top of the shaft. To preserve the limited space on the ground level of the building, the initial conceptual design explores placing this supporting equipment on a dedicated platform over the doors to the building's mechanical room.

- Power and grounding considerations need to be taken into account. It is expected that a clean ground will be required as well as power conditioning.

- A database will be needed to store ambient data from the surroundings. This will include temperature, pressure and humidity, local weather, vibration and electromagnetic field variations. The data will be time-stamped to allow correlations both with the experimental data and with external records. 


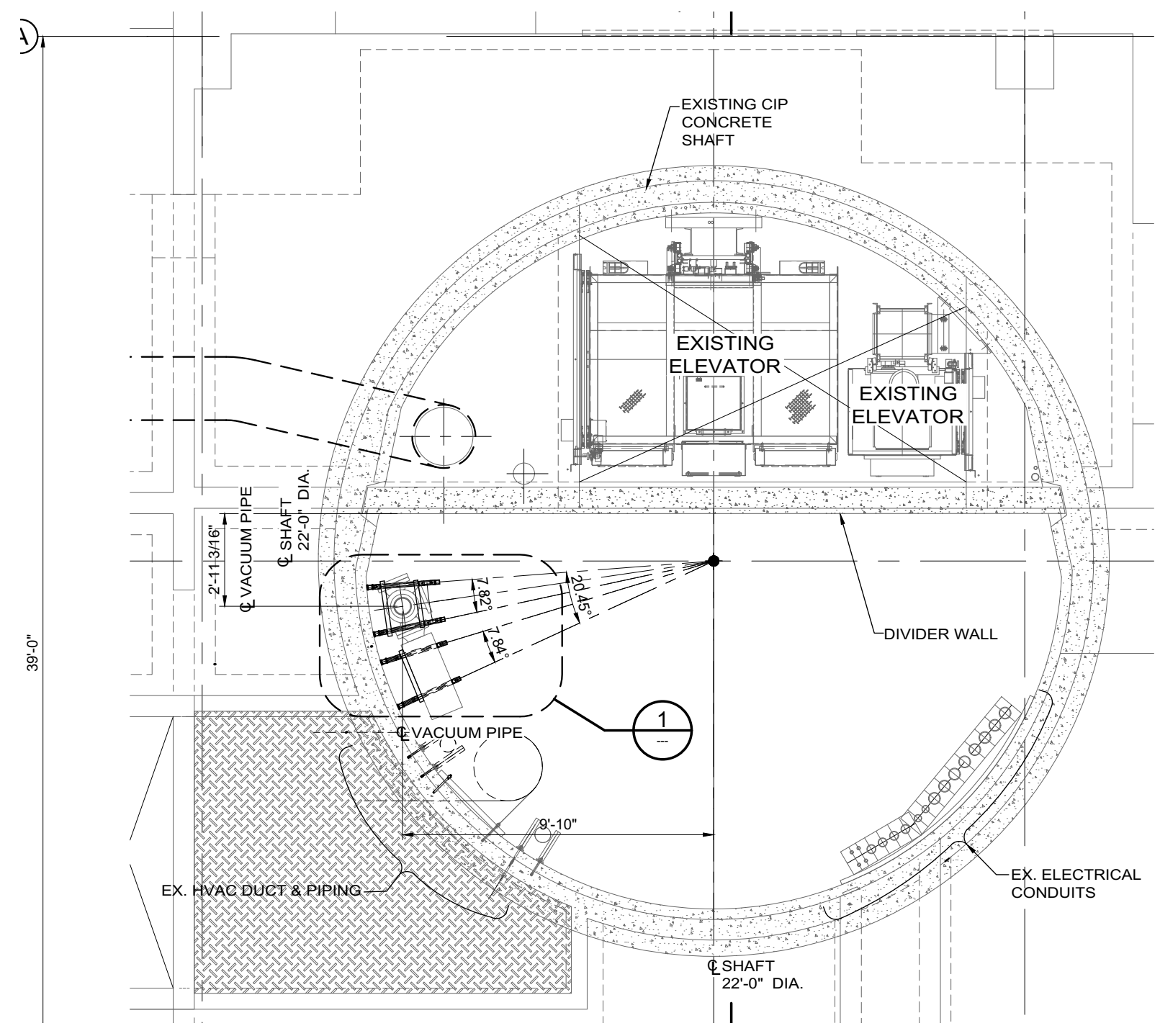

Figure 15: Plan view showing location of MAGIS-100 vacuum pipe within the shaft. 


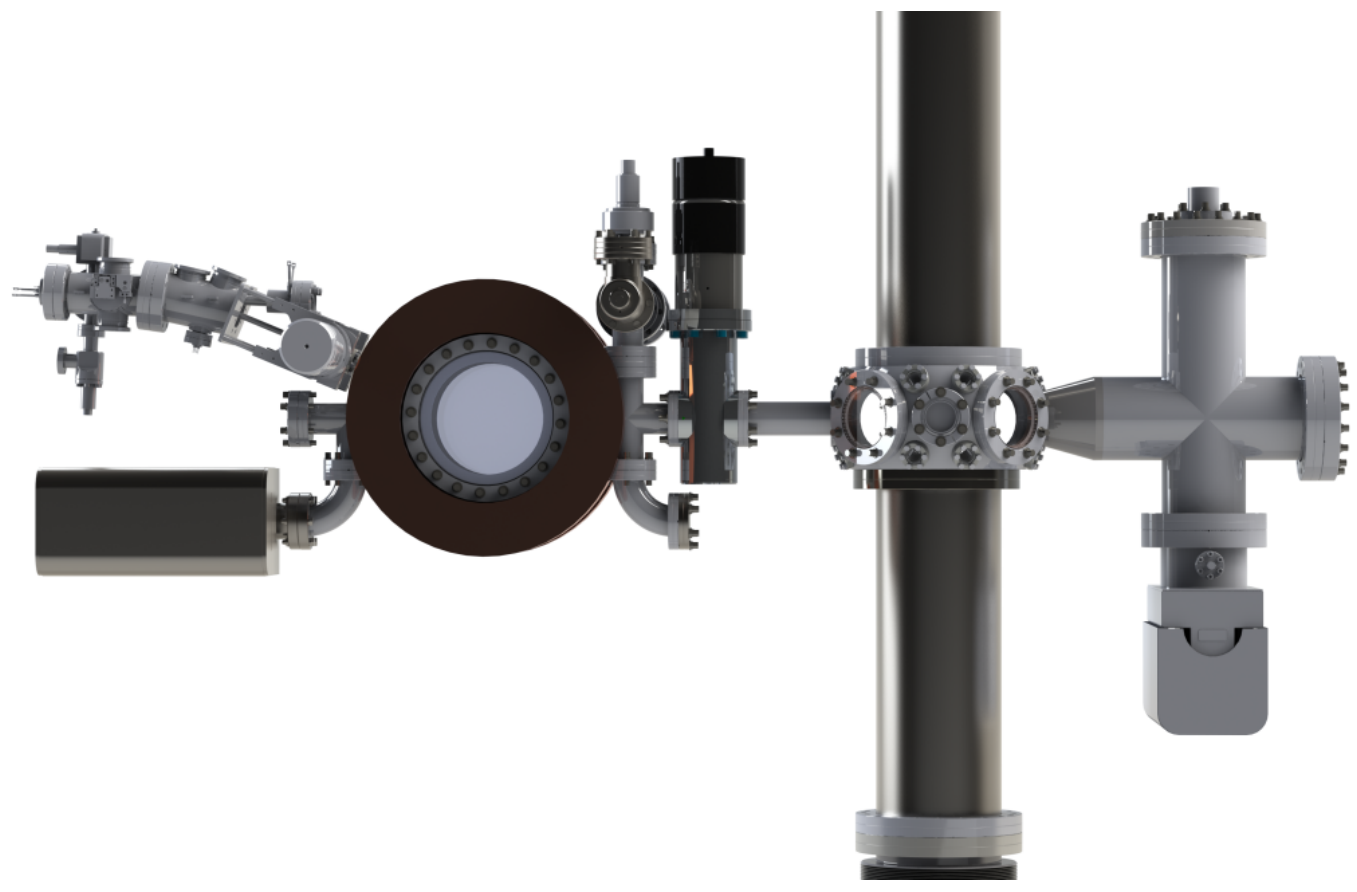

Figure 16: Atom source detail. CAD model of one of the three atom sources. Each atom source consists of a $\mathrm{Sr}$ beam source and a laser cooling vacuum chamber (left) that is connected to the vertical vacuum pipe (right of center), as well as the lasers, optics, and magnetic coils required for atom cooling and trapping, and vacuum pumps (right). For clarity, the optics frame surrounding the cooling chamber is not shown. 


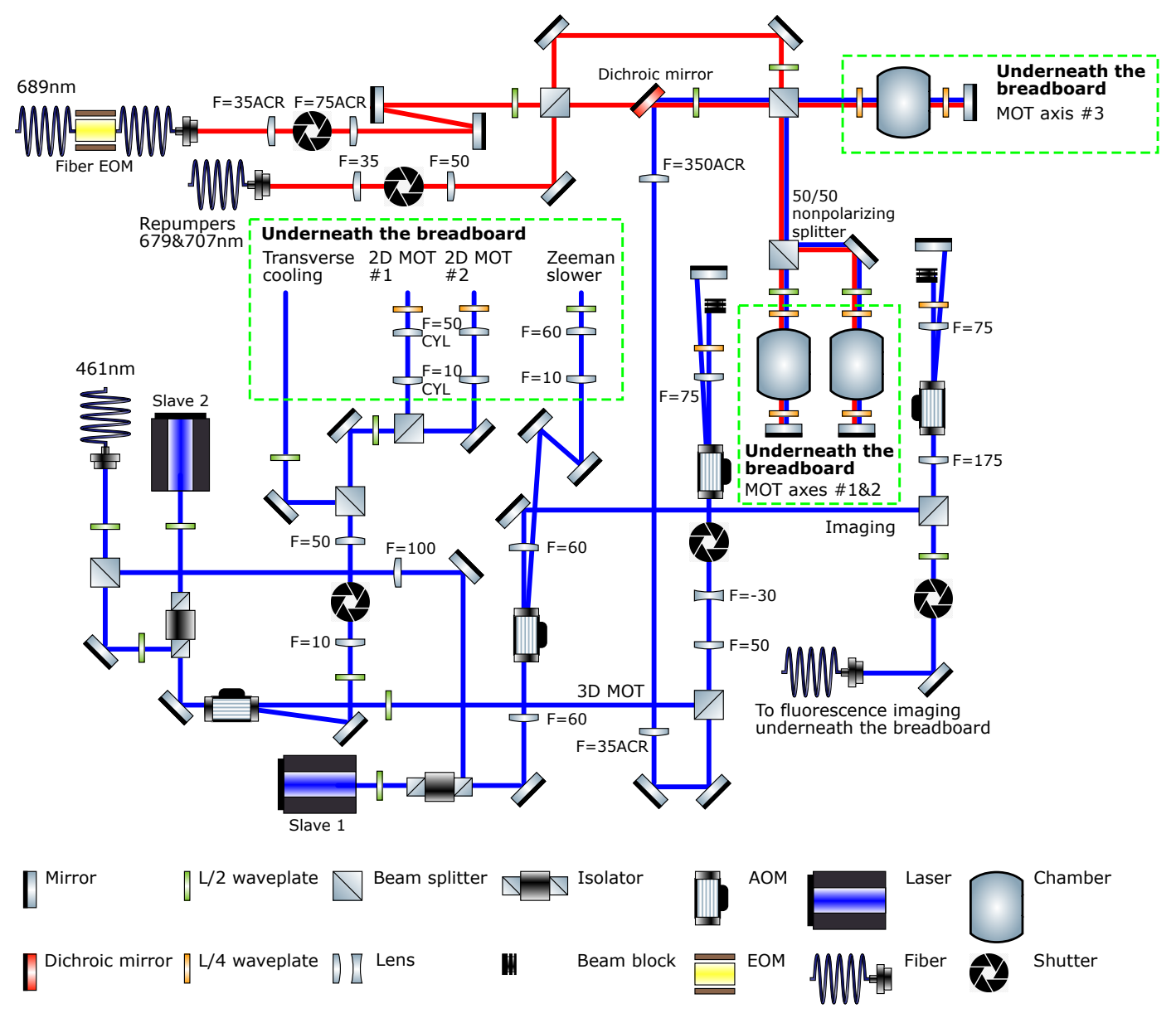

Figure 17: Atom source optics layout. The optics breadboard for the $461 \mathrm{~nm}$ and $689 \mathrm{~nm}$ laser cooling light is located inside the atom sources and delivers the light to the MOT vacuum chamber and beam source chamber. 


\subsection{Characterizing the environment}

An assessment of vibrations using a precision seismometer will be done to inform the final design. Data will be gathered over a period of days to weeks to make sure representative vibration sources are sampled. In particular, systematic measurements will be made at the location of the laser enclosure and its optics. Preliminary measurements of the vibration spectrum are shown in Fig. 18. Major known sources of machinery noise are the sump pumps, which must function at all times to prevent flooding in the underground area. Additionally, the shaft elevator is a rack-and-pinion system, and is located just behind the east shaft wall. Both these sources are intermittent, and will probably be gated out or removed during off-line data processing. Ongoing vibration monitoring is expected. Measurements will be taken at the top of the shaft, and, if possible, at a location in the shaft near the bottom of the system.

It is planned to perform a magnetic survey of the shaft with flux gate magnetometers, and follow this with a system of magnetic monitors. A preliminary assessment has been carried out using a hand-held probe from the g-2 experiment (Bartington Instruments Mag690 3-axis flux gate magnetometer), the results are shown in Fig. 19. In the longer term, a dedicated multiple station setup is planned. The goal is to monitor several equally spaced points along the length of the shaft and near the optical enclosures. The monitors will be mounted to the shaft on non-magnetic supports. To minimize development time, we expect to use a variant of the system used by the g-2 experiment when feasible, based on inexpensive 3-axis fluxgate magnetometers (Bartington Mag690).

Use of the MINOS building for its utility purposes may result in a "wall" of cold air in the winter, in addition to year round temperature variance from the top of the shaft to the bottom. In particular, opening the large roll-up door in the MINOS building in cold weather causes a sudden several degree temperature drop in the area at the top of the shaft. Presently there is a plan to monitor the environment with a temperature logging monitor at several points in the hall, and at several equally spaced points in the NuMI shaft. It is foreseen that the three atomic sources for the experiment, and the interferometry laser, will be housed in thermally controlled enclosures.

\subsection{Other site-specific considerations}

- Water inflow mitigation. The MINOS shaft is a damp environment, particularly during wet weather, when there is inflow via building penetrations, as well as modest water leakage at several points within the shaft. There is the need to identify requirements and options for sealing against water, and options for making modest improvements to the building penetrations.

- Power and Networking. It is foreseen that power will need to be routed into the optical enclosures for the interferometry beams, as well as the atom sources at several points in the shaft. Power will be required for the active magnetic compensation around the vacuum chamber, and other components such as heaters and vacuum pumps. Networking to the building is available due to the requirements of the NuMI experiments. Network and other control cables will run from a rack or racks at the top of the shaft down the full height of the apparatus.

- Magnetic shielding. Magnetic shielding will be required to prevent ambient fields from affecting the measurements. The shielding should completely envelop the system and will be external to the bias field created outside the vacuum pipe. The level of shielding required will depend in part on the results of the site characterization. 


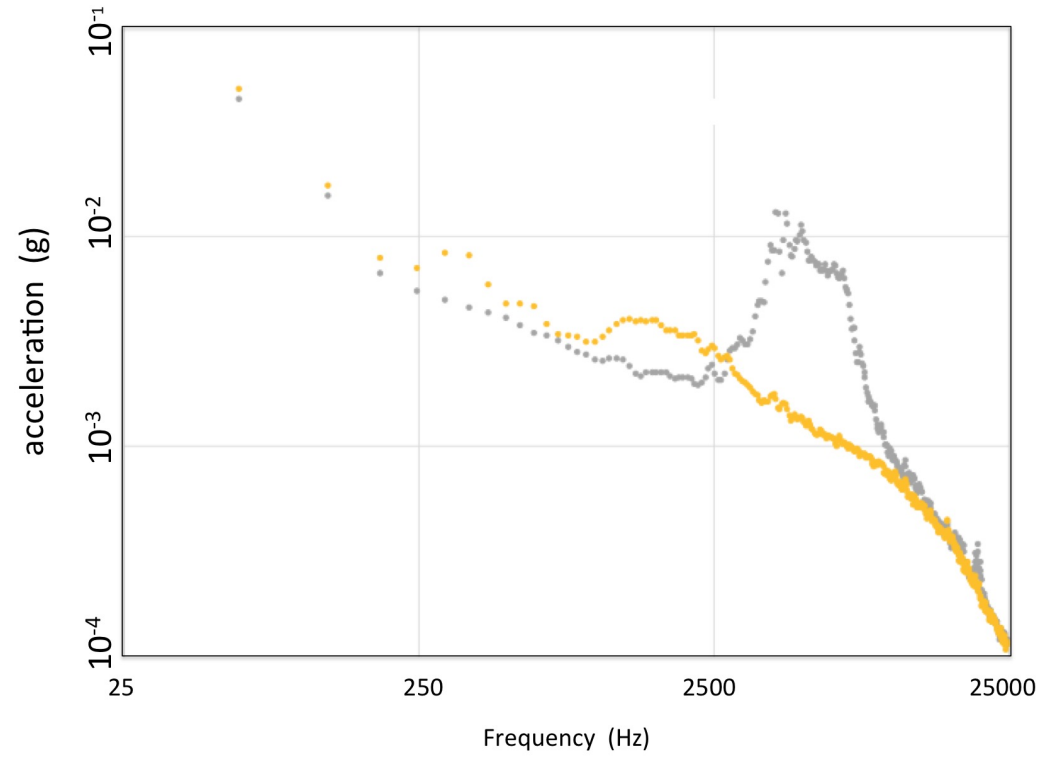

Figure 18: Preliminary vibrational spectrum at top (orange) and bottom (grey) of shaft east wall (elevator side). The peak at approx. $3000 \mathrm{~Hz}$ may represent machinery noise. (In the plot, the $\mathrm{x}$-axis is in units of $\mathrm{Hz}$, and the $\mathrm{y}$-axis in units of gravitational acceleration $\mathrm{g}$.

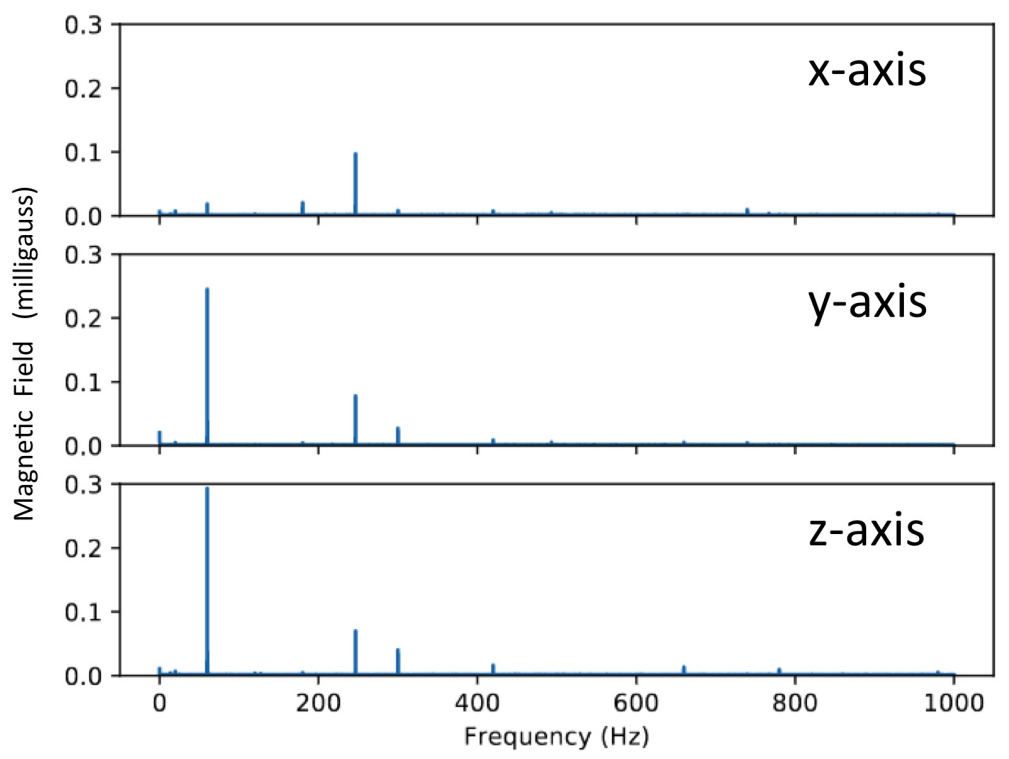

Figure 19: Initial measurement of time-varying magnetic field strength at the top of the MINOS shaft. Visible are the expected $60 \mathrm{~Hz}$ harmonics and a line of unknown origin at $246.6 \mathrm{~Hz}$

- Vacuum system. The vacuum pressures required for MAGIS-100 have previously been achieved at Fermilab in the Recycler Ring during the antiproton program. For MAGIS-100 the vacuum requirement is reasonable although challenging. Based on experience at Fermilab, ion pumps combined with either titanium sublimation pumps (TSP's) or non-evaporable getter 
(NEG) pumps or coatings will be used. A vacuum bake system is required to achieve the desired pressure and must be designed with non-magnetic materials or with parts that can be easily removed to prevent magnetic interference. Bellows will be designed for both installation alignment and to allow thermal expansion during bakes. Mechanical components will be typical for ultra-high-vacuum (UHV) systems previously used at Fermilab. A blackout coating may be applied to the vacuum pipe to improve optics. The initial concept is to use Aerodag, which can be used in UHV if it is baked after application. There is experience at Fermilab using this material in kicker tubes for a different purpose.

- Control and Monitoring. There is space for an electrical rack at the top of the shaft with controllers for the ion pumps, gauges, and valves. To facilitate control, monitoring, and data logging, these devices will be connected to ACNET. For the central control and monitoring area, we anticipate using space in ROC West on the ground floor of Wilson Hall.

- Safety and shaft infrastructure. An existing personnel basket attaches to the crane for emergency egress from the underground enclosure. The MAGIS equipment will be designed to preserve this functionality, and it will also utilize the basket for installation and maintenance. A mechanical cage will be built around the equipment to provide rudimentary bump protection from objects being lowered into the shaft with the crane. The sources and vacuum tube will be anchored to the wall of the shaft with conventional concrete anchors. The design of the vacuum tube supports must allow rolling motion for thermal expansion of the tube while maintaining a rigid connection and not allowing the system to elongate due to gravity.

\subsection{Error Modeling}

Error modeling for MAGIS-100 is based on extensive, previously published analysis of noise backgrounds for differential atom interferometers $[21-23,58,69]$. The relevant sources of noise for MAGIS-100 include: laser frequency noise [23], laser wavefront aberrations [22,60], seismic vibration [1, 23], Coriolis effects arising from Earth's rotation [21, 22, 60, 61], laser pointing jitter [22,60,61,69,70], AC Stark shifts [17], initial cloud kinematics [12, 21, 22, 60,61], mean field shifts [71], magnetic fields [22,63,64,69], blackbody radiation shifts [64,69], and imaging aberrations $[1,12]$. These effects are all well-understood (see the above references), and preliminary studies of many of them have been made in the Stanford $10 \mathrm{~m}$ fountain [1,12,17,60,61]. Appendix $\mathrm{H}$ contains a more detailed discussion of the sources of systematic uncertainty for MAGIS-100 and the experimental strategies for mitigating their impact.

\section{Development plan}

\begin{tabular}{|l|c|c|c|}
\hline Sensor technology & State of the art & Goal & GW sensitivity improvement \\
\hline LMT atom optics & $n=10^{2}$ & $n=10^{3}$ & 10 \\
\hline Spin squeezing & $20 \mathrm{~dB}(\mathrm{Rb}), 0 \mathrm{~dB}(\mathrm{Sr})$ & $20 \mathrm{~dB}(\mathrm{Sr})$ & 10 \\
\hline Atom flux & $\sim 10^{6}$ atoms $/ \mathrm{s}$ & $10^{8}$ atoms $/ \mathrm{s}$ & 10 \\
\hline
\end{tabular}

Table 1: Research and development goals.

The MAGIS-100 science program can be thought of as starting with a set of initial goals, characterized for example by the blue dashed curve in Fig. 4, and ending with a set of stretch goals, 


\begin{tabular}{|c|c|c|}
\hline & Task & Description \\
\hline \multirow{2}{*}{$\stackrel{+1}{\check{\varpi}}$} & Design high flux source & $\begin{array}{l}\text { Design will address increasing thermal beam flux (oven and } \\
\text { Zeeman slower) and cooling in multiple stage MOT cham- } \\
\text { bers }\end{array}$ \\
\hline & Design squeezed source & $\begin{array}{l}\text { Perform design trades and preliminary laboratory investi- } \\
\text { gations for squeezed-state metrology. }\end{array}$ \\
\hline \multirow{3}{*}{ 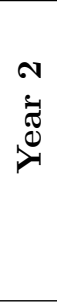 } & Composite pulses & $\begin{array}{l}\text { Demonstrate composite pulses on the Sr clock transition in } \\
\text { an LMT interferometer. }\end{array}$ \\
\hline & $\begin{array}{l}\text { Construct high flux } \\
\text { source apparatus }\end{array}$ & $\begin{array}{l}\text { Qualify thermal beam modifications. Assemble vacuum } \\
\text { chamber and laser cooling optics. }\end{array}$ \\
\hline & $\begin{array}{l}\text { Construct squeezed } \\
\text { source module }\end{array}$ & $\begin{array}{l}\text { Build and test squeezed source module. Demonstrate } 10 \\
\text { dB state-squeezing. }\end{array}$ \\
\hline \multirow{4}{*}{$\underset{\infty}{\infty}$} & $\begin{array}{l}\text { Resonant } \\
\text { interferometry }\end{array}$ & $\begin{array}{l}\text { Use LMT techniques to make a resonant interferometer } \\
\text { with a resonant enhancement of } 10 .\end{array}$ \\
\hline & AC Stark suppression & $\begin{array}{l}\text { Study AC Stark suppression techniques for LMT interfer- } \\
\text { ometer (spectral engineering, intensity control, etc.) }\end{array}$ \\
\hline & $\begin{array}{l}\text { Increased steady-state } \\
\text { atom number }\end{array}$ & $\begin{array}{l}\text { Demonstrate improved steady-state atom number in high } \\
\text { flux source. Target is } 10^{9} \text { atoms in red MOT. }\end{array}$ \\
\hline & $\begin{array}{l}\text { Integrate squeezed } \\
\text { source }\end{array}$ & $\begin{array}{l}\text { Integrate squeezed-state source into interferometry appara- } \\
\text { tus. Demonstrate squeezed state interferometry. }\end{array}$ \\
\hline \multirow{3}{*}{ 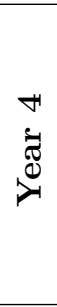 } & $\begin{array}{l}\text { Demonstrate } 10^{8} \\
\text { atoms/s atom flux }\end{array}$ & Extract atoms from high phase space density (red) MOT. \\
\hline & $\begin{array}{l}\text { Levitated } \\
\text { interferometer }\end{array}$ & $\begin{array}{l}\text { Demonstrate a levitated interferometer proof of principle } \\
\text { for }>100 \mathrm{~ms} \text { using the Sr clock transition. }\end{array}$ \\
\hline & $\begin{array}{l}\text { Squeezed state } \\
\text { interferometry }\end{array}$ & $\begin{array}{l}\text { Demonstrate } 10 \mathrm{~dB} \text { state squeezing with LMT interferom- } \\
\text { etry. }\end{array}$ \\
\hline \multirow{3}{*}{ ¿্ं } & $\begin{array}{l}\text { Demonstrate } 10^{8} \\
\text { atoms/s at target } \\
\text { temperature }\end{array}$ & $\begin{array}{l}\text { Incorporate evaporative cooling and/or matter wave lensing } \\
\text { protocols to reduce temperature to } 10 \mathrm{nK} \text { range. }\end{array}$ \\
\hline & LMT Wavefront & $\begin{array}{l}\text { Incorporate wavefront noise mitigation strategies in LMT } \\
\text { interferometry (including wavefront characterization and } \\
\text { feedback) }\end{array}$ \\
\hline & $\begin{array}{l}\text { Precision squeezed state } \\
\text { interferometry }\end{array}$ & $\begin{array}{l}\text { Demonstrate } 20 \mathrm{~dB} \text { state squeezing with high atom flux and } \\
\text { LMT interferometry. }\end{array}$ \\
\hline
\end{tabular}

Table 2: Tasks by performance year for R\&D program which will be conducted at Stanford. 
characterized for example by the MAGIS-100 (5-year) line in Fig. 4. The initial design parameters for MAGIS-100, as well as upgrade goals, are summarized in Table 1. The R\&D will be organized into three parallel efforts: developing advanced atom optics (large momentum transfer techniques), designing high-flux atom sources, and producing spin-squeezed sources (entanglement). A year-byyear list of these R\&D activities is shown in Table 2.

After achieving the initial R\&D goals listed in Table 1, the ultimate MAGIS-100 R\&D goal is to advance atom interferometer detector technology toward the point where the construction of a kilometer-scale detector is possible at the sensitivity level shown in Fig. 4. This will require further increasing LMT to the level of $n \approx 10^{4}$ or making a corresponding improvement in phase resolution via higher atom flux or improved squeezing.

\subsection{Large momentum transfer atom optics}

The detector benefits from advanced large momentum transfer (LMT) atom optics. An LMT enhanced interferometer uses multiple laser pulses to increase the sensitivity of the detector (sensitivity grows linearly with the number of laser pulses). Existing state-of-the-art interferometers achieve LMT enhancement factors of around $n \sim 100$ [17], limited in part by unwanted scattering (spontaneous emission). Atom interferometry performed on the clock transition in Sr has comparatively negligible spontaneous emission loss compared to existing approaches and could allow for a significant increase in the efficiency of atom-light interactions. For typical pulse parameters, this loss rate is small enough to allow $>10^{6}$ single photon transitions, substantially more than the $\sim 10^{2}$ sequential two-photon Bragg transitions that are possible with current generation laser systems [17]. To combat laser technical noise limitations, reduced spontaneous emission opens up the possibility of using techniques borrowed from NMR such as composite pulse sequences [72] to increase pulse efficiency in the presence of such errors. To reach the target of $n \sim 10^{3}$ pulses will require investigation into other atom loss processes, improved atom cooling, as well as further laser technical improvements.

\subsection{High flux atom sources}

The phase resolution of the interferometer is ultimately limited by the finite number of atoms in the ensemble, typically $10^{5}-10^{6}$. Quantum projection noise (shot noise) in the phase readout can be reduced by using a larger number of atoms in each measurement. High flux commercial beam sources can now provide $>10^{12}$ atoms/s, though the atoms are too hot to use directly. Work will focus on harnessing such high flux sources by developing cooling protocols to reach the necessary low temperatures while maintaining high atom numbers, targeting $10^{8}$ cold atoms per ensemble. Preliminary progress has been made delivering high phase-space density Sr ensembles in steady-state using a series of separated traps [73]. A version of this approach will be explored that integrates a high flux beam source.

\subsection{Spin-squeezed sources}

The shot noise limit can be avoided by correlating the atoms in the ensemble using quantum entanglement ('spin squeezing'). State-of-the-art squeezed sources using alkali-atoms have demonstrated up to $20 \mathrm{~dB}$ of squeezing using Rb atoms (implying a factor of 10 noise reduction in the sensor compared to a conventional source) $[18,74]$. The methods demonstrated in this prior work translate directly to the MAGIS detector. Proposed work will focus on transferring this technique to Sr atoms and demonstrating a squeezed inertial sensor that could be integrated with the MAGIS-100 platform. Technical challenges include extending the interrogation times available for squeezed states 


\begin{tabular}{|c|c|c|c|c|c|}
\hline & Institution & Scientists & Postdocs & Students & $\begin{array}{l}\text { TOTAL } \\
\text { FTEs }\end{array}$ \\
\hline \multirow{7}{*}{ ت } & Berkeley & 0.2 & 0 & 0.5 & 0.7 \\
\hline & Fermilab & 1.7 & 0 & 0 & 1.7 \\
\hline & Liverpool & 0.5 & 0 & 0 & 0.5 \\
\hline & NIU & 0.5 & 0 & 1 & 1.5 \\
\hline & Northwestern & 0.3 & 1 & 1 & 2.3 \\
\hline & Stanford & 0.5 & 2 & 4 & 6.5 \\
\hline & Total & 3.7 & 3.0 & 6.5 & 13.2 \\
\hline \multirow{7}{*}{ 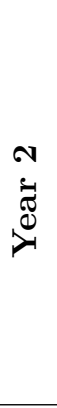 } & Berkeley & 0.2 & 0 & 0.5 & 0.7 \\
\hline & Fermilab & 1.7 & 1 & 0 & 2.7 \\
\hline & Liverpool & 0.5 & 1 & 1 & 2.5 \\
\hline & $\mathrm{NIU}$ & 0.5 & 0 & 2 & 2.5 \\
\hline & Northwestern & 0.3 & 1 & 1 & 2.3 \\
\hline & Stanford & 0.5 & 2 & 4 & 6.5 \\
\hline & Total & 3.7 & 5.0 & 8.5 & 17.2 \\
\hline \multirow{7}{*}{$\begin{array}{l}\infty \\
\dot{\varpi ే} \\
\stackrel{\nexists}{\beth}\end{array}$} & Berkeley & 0.2 & 0 & 0.5 & 0.7 \\
\hline & Fermilab & 1.7 & 1 & 0 & 2.7 \\
\hline & Liverpool & 0.5 & 1 & 2 & 3.5 \\
\hline & $\mathrm{NIU}$ & 0.7 & 1 & 2 & 3.7 \\
\hline & Northwestern & 0.3 & 1 & 1 & 2.3 \\
\hline & Stanford & 0.5 & 1 & 4 & 5.5 \\
\hline & Total & 3.9 & 5.0 & 9.5 & 18.4 \\
\hline
\end{tabular}

Table 3: Anticipated evolution of the scientific effort (FTEs) of the MAGIS-100 Collaboration during construction, installation and commissioning. Note that the anticipated estimate of effort at Liverpool is dependent on the development of funding opportunities in the U.K.

(currently 10 msec, unpublished) and developing methods suitable for large ensembles of atoms. In particular, entangled Sr ensembles will be created and detected with optical cavity-assisted quantum non-demolition measurements.

\section{Responsibilities, resource requirements, and timeline}

\subsection{Responsibilities}

MAGIS-100 is a collaborative effort which brings together the atom source and interferometry expertise of university collaborators with the engineering and technical resources offered by a large laboratory. The contributions and responsibilities of each institutional group are summarized in Appendices A-F. The anticipated year-by-year scientific effort to carry out these institutional responsibilities is summarized in Table 3. Note that after three years, we anticipate that the scientific effort will have ramped up to about 18 FTEs. We believe this is adequate to be able 


\begin{tabular}{|c|c|c|c|}
\hline & Task & Description & Location \\
\hline \multirow{4}{*}{ ri } & $\begin{array}{l}\text { Atom source design and } \\
\text { procurement }\end{array}$ & $\begin{array}{l}\text { Adapt existing designs and add environmental protection } \\
\text { and other hardware needed to integrate into MAGIS- } 100 .\end{array}$ & Stanford \\
\hline & $\begin{array}{l}\text { Laser system design } \\
\text { and procurement }\end{array}$ & $\begin{array}{l}\text { Design high-power atom optics laser system based on co- } \\
\text { herently combined Ti:sapphire lasers. Procure necessary } \\
\text { equipment. }\end{array}$ & Stanford \\
\hline & $\begin{array}{l}\text { Preliminary site } \\
\text { engineering }\end{array}$ & $\begin{array}{l}\text { Study vibration environment, magnetic field environment, } \\
\text { and temperature environment. Begin engineering for vibra- } \\
\text { tion isolation (if necessary), magnetic shielding and active } \\
\text { magnetic field compensation, and temperature control. }\end{array}$ & Fermilab \\
\hline & $\begin{array}{l}100 \mathrm{~m} \text { vacuum vessel } \\
\text { design and procurement }\end{array}$ & $\begin{array}{l}\text { Design system of vacuum pumps, viewports, and atom } \\
\text { source connection nodes. Procure necessary equipment. }\end{array}$ & $\begin{array}{l}\text { Stanford/ } \\
\text { FNAL }\end{array}$ \\
\hline \multirow{4}{*}{$\stackrel{N}{\stackrel{\varpi}{0}}$} & $\begin{array}{l}\text { Build } 100 \mathrm{~m} \text { vacuum } \\
\text { segments }\end{array}$ & Install viewports and connection nodes. & Stanford \\
\hline & Complete site design & Finalize vibration, magnetic, and temperature engineering. & Fermilab \\
\hline & $\begin{array}{l}\text { Atom source } \\
\text { qualification }\end{array}$ & $\begin{array}{l}\text { Build atom sources. Verify that necessary atom flux is de- } \\
\text { livered. }\end{array}$ & Stanford \\
\hline & $\begin{array}{l}\text { Laser system } \\
\text { qualification }\end{array}$ & $\begin{array}{l}\text { Build laser system. Verify that power delivered, frequency } \\
\text { and amplitude agility, and phase noise meet specifications. }\end{array}$ & Stanford \\
\hline \multirow[t]{2}{*}{$\stackrel{\infty}{\infty}$} & Detector commissioning & $\begin{array}{l}\text { Install } 100 \mathrm{~m} \text { vacuum vessel, magnetic shield, atom sources, } \\
\text { and laser system. Test lattice shuttling of atoms from atom } \\
\text { sources into } 100 \mathrm{~m} \text { vacuum tube, dropping of atoms, lattice } \\
\text { launching of atoms, and atom optics laser pulses. }\end{array}$ & Fermilab \\
\hline & $\begin{array}{l}\text { Atom interferometry in } \\
100 \text { meter vacuum }\end{array}$ & $\begin{array}{l}\text { Run atom interferometers using each of the three atom } \\
\text { sources. Implement LMT atom optics in interferometers. }\end{array}$ & Fermilab \\
\hline \multirow{2}{*}{ 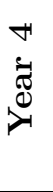 } & $\begin{array}{l}\text { Gradiometer with two } \\
\text { sources }\end{array}$ & Long baseline gradiometer. Study noise sources. & Fermilab \\
\hline & Science data runs & $\begin{array}{l}\text { Long science data runs with two-source gradiometer (grav- } \\
\text { itational wave detector prototype). }\end{array}$ & Fermilab \\
\hline \multirow{2}{*}{ 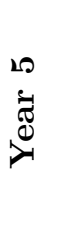 } & $\begin{array}{l}\text { Gradiometer with three } \\
\text { sources }\end{array}$ & Incorporate third source into long baseline gradiometer. & Fermilab \\
\hline & Study GGN suppression & $\begin{array}{l}\text { Use three-source gradiometer to study gravity gradient } \\
\text { noise (GGN) impact and mitigation strategies. Additional } \\
\text { long science data runs. }\end{array}$ & Fermilab \\
\hline
\end{tabular}

Table 4: Tasks by performance year for MAGIS-100 prototype 
to analyze the data and operate MAGIS-100 24 hours a day, 7 days a week when needed for production running. For comparison, in steady operations the $10 \mathrm{~m}$ baseline experiment at Stanford has a scientific staff of 3.3 FTEs (0.3 FTE scientists, 2.0 FTE postdocs, 1.0 FTE students). At the proposal stage, the present effort is concentrated at Stanford and Fermilab. The growth in the level of effort at these institutions will be supported by, respectively, the existing Moore Foundation funding and the request for support of the Fermilab effort that will be made to DOE-OHEP. The growth of effort at the other institutions will take place as opportunities for support become available.

The main year-by-year tasks, and the location of the associated activities, are listed in Table 4. The principal parts for MAGIS-100 will be procured and qualified at Fermilab and Stanford, with other collaborating institutions contributing as necessary. Procurement and assembly of the atom sources will be carried out at Stanford in identified laboratory space. This leverages the very significant atomic physics expertise of the Stanford group. In addition, procurement and initial qualification of the vacuum chamber components, and procurement and qualification of the interferometry laser system, will be overseen via Stanford. Fermilab has responsibility for infrastructure, including the support structure for the vacuum system and atom sources in the MINOS shaft, and support structures for the interferometry lasers, as well as necessary utilities.

Fermilab labor resources are required for engineering, technical completion of the systems (particularly the vacuum pipe system), and assembly. The balance between engineering and technical effort changes from year one (mostly engineering) to the other years (mostly technical). Significant drafting resources are needed in year one.

\subsection{Procurements}

The major equipment procurements needed for MAGIS-100 are:

- Custom atom sources: These are at the heart of the interferometry system. The purchased base system is to be modified using the expertise of MAGIS-100 university collaborators (currently Stanford). This includes the laser system need for cooling and trapping the atoms and transporting them (optical lattice shuttle and launch) to the $100 \mathrm{~m}$ vacuum chamber.

- $100 \mathrm{~m}$ vacuum chamber: This is an ultra-high-vacuum stainless steel pipe with heaters, advanced pumping, magnetic shielding, and magnetic bias coils to facilitate the atomic spectroscopy.

- Shaft support structure: It is anticipated that an industrial system can be used for the supports that fasten the vacuum system/atom source assemblies to the concrete wall of the MINOS shaft.

- Laser hutch and support platform: This is conventional construction as performed elsewhere at Fermilab.

- Installation: Both a rigging team and additional gantry crane will be required. The principal crane will be used for personnel access.

\subsection{Utilization of laboratory infrastructure}

We expect to utilize the following laboratory infrastructure:

- Building maintenance and services. 
- Remote Operations Center (ROC) maintenance and services.

- Support of sitewide computing network.

- Support of accelerator controls network.

- Safety and environmental support.

\subsection{Cost Estimate}

Estimated costs for constructing and installing MAGIS-100 are summarized in Table 5. Note that $50 \%$ of the cost is based on physicist-level estimates and $50 \%$ of the costs are based on engineeringlevel estimates. In general, the physicist estimates utilize preliminary item counts and expertise from performing similar work currently and in the recent past. Engineering estimates are primarily for work at FNAL and are generated by engineering staff with experience in similar work, e.g. vacuum systems and support structure construction. In each case detailed item counts are being maintained and adjusted as our knowledge of the design improves.

\begin{tabular}{|c|c|c|}
\hline & & $\begin{array}{l}\text { Direct } \\
\text { Cost }(\$)\end{array}$ \\
\hline \multirow{4}{*}{ 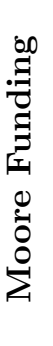 } & $\begin{array}{l}\text { Fabricate Atom Sources } \\
\text { (Stanford) }\end{array}$ & $1,869,000$ \\
\hline & $\begin{array}{l}\text { Vacuum Tube } \\
\text { Procurement (Stanford) }\end{array}$ & 931,060 \\
\hline & $\begin{array}{l}\text { Interferometer Laser } \\
\text { (Stanford) }\end{array}$ & 593,200 \\
\hline & Sub-Total & $3,393,260$ \\
\hline \multirow{8}{*}{ 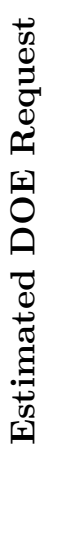 } & $\begin{array}{l}\text { Interferometer Shaft } \\
\text { Support Structure }\end{array}$ & 99,000 \\
\hline & $\begin{array}{l}\text { Laser hutch and support } \\
\text { platform }\end{array}$ & 21,000 \\
\hline & Engineering (FNAL) & 367,000 \\
\hline & Drafting and Technical & 304,000 \\
\hline & Installation Equipment & 28,000 \\
\hline & Installation & 51,000 \\
\hline & Operation and Materials & 49,000 \\
\hline & Sub-Total & 919,000 \\
\hline 焉 & $\begin{array}{l}\text { DIRECT } \\
\text { INDIRECT } \\
\text { FULLY BURDENED }\end{array}$ & $\begin{array}{l}4,312,260 \\
691,000 \\
5,003,260\end{array}$ \\
\hline
\end{tabular}

Table 5: Preliminary estimate of the equipment and technical personnel costs during the three-year MAGIS-100 construction and installation period. The expected source of funding for major items is also indicated. 


\begin{tabular}{|l|r|}
\hline Direct Costs & \\
Personnel & $4,503,459$ \\
Travel & 23,905 \\
M\&S for R\&D & 374,000 \\
M\&S for MAGIS-100 & 682,200 \\
Equipment for R\&D & $1,124,000$ \\
Equipment for MAGIS-100 & $2,711,060$ \\
\hline Total Direct & $\mathbf{9 , 4 1 8 , 6 2 4}$ \\
Indirect & 398,608 \\
\hline TOTAL $\mathbf{( \$ )}$ & $\mathbf{9 , 8 1 7 , 2 3 2}$ \\
\hline
\end{tabular}

Table 6: Summary of Funding from the Moore Foundation.

\begin{tabular}{|l|r|}
\hline M\&S for MAGIS-100 & 682,200 \\
Equipment for MAGIS-100 & $2,711,060$ \\
\hline Total $(\$)$ & $\mathbf{3 , 3 9 3 , 2 6 0}$ \\
\hline
\end{tabular}

Table 7: Summary of the MAGIS-100 specific part of the funding (direct costs) from the Moore Foundation.

The costs shown in Table 5 are separated by major subsystems. The funding sources (Moore Foundation grant that is in place and the anticipated request to the DOE to support Fermilab related activities and responsibilities) are also indicated for each item. Table 6 summarizes the Moore Foundation grant and, for clarity, Table 7 shows the total MAGIS-100 specific part of that funding.

The subsystems listed in Table 5 will map smoothly onto the experiment WBS, which is in preliminary development. The estimate assumes construction and deployment over a three-year period. Funding for subsequent operations will be treated separately. Indirect costs vary by institution, but are included at a level correct for each subsystem. Support for the scientific effort, described in section 9.1, is not included, however the engineering and technical effort is included in the table.

\subsection{Technically Driven Timeline}

We propose a three year construction, installation and commissioning plan, shown in Fig. 20. Major milestones are shown in Table 8. Note that a pair of prototype Sr atom sources and an associated atom interferometry laser system are already being constructed and tested at Stanford. These atom sources will serve as the baseline design for MAGIS-100, allowing us to take advantage of proven atom source technology. The prototype sources have achieved laser cooled $\mathrm{Sr}$ atoms, and atom interferometry tests will be performed shortly. An image of resonantly scattered light from a cold Sr atom cloud in the Stanford setup is shown in Fig. 21.

Expected activities in the three-year construction and installation period are summarized as follows. In year 1 , the $\sim 100 \mathrm{~m}$ vacuum pipe will be designed by FNAL, in consultation with Stanford. The atom source and atom interferometer laser will be designed and their assembly will begin at Stanford. In year 2, FNAL will complete site characterization, and pre-assembly 


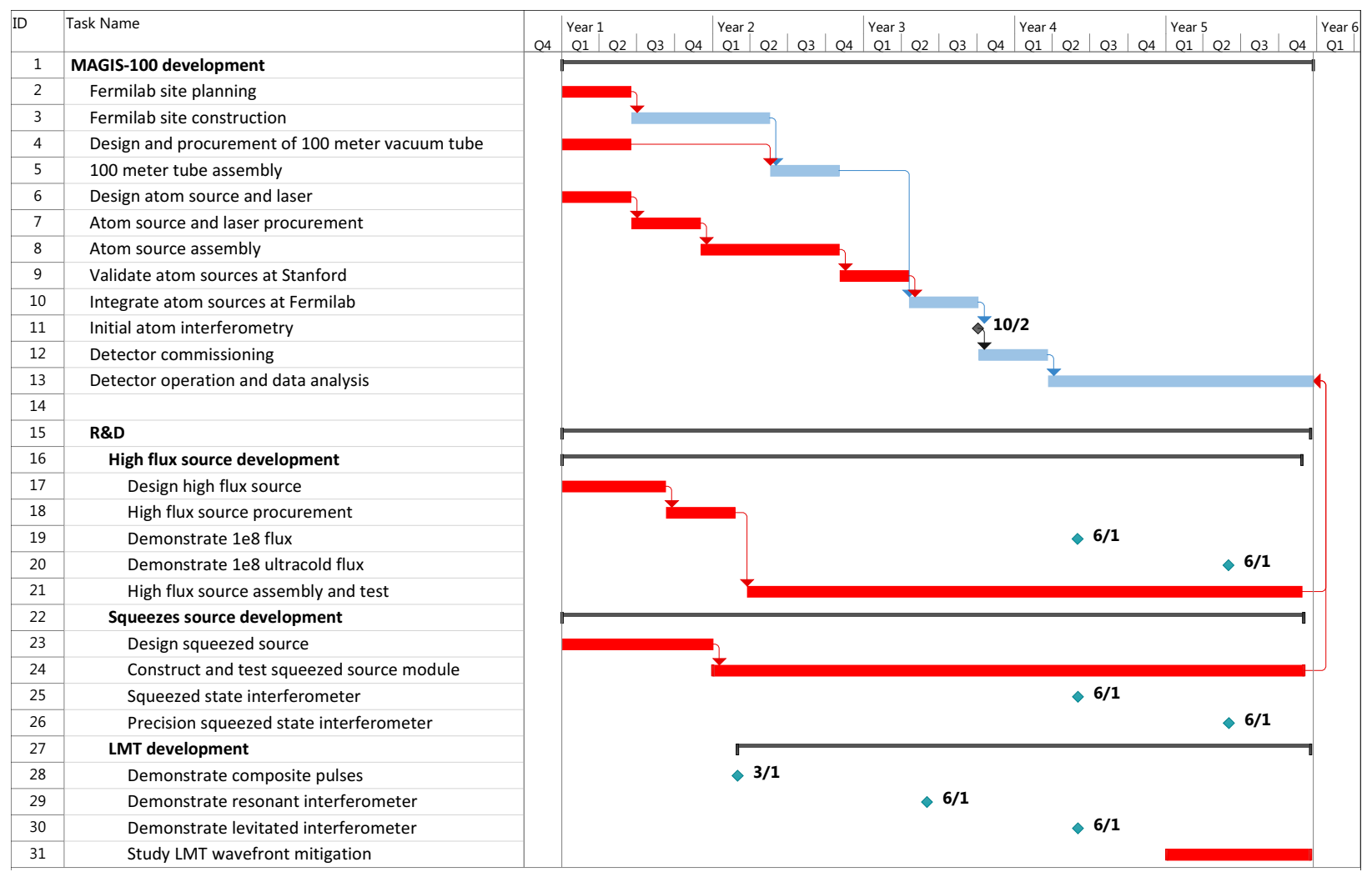

Figure 20: Technically driven timeline. Blue: Activities at Fermilab. Red: Activities at Stanford. Ongoing R\&D program in source development also shown.

of the vacuum pipe components. Stanford will complete construction and initial testing of the atom source and interferometry laser. The atom source and laser will then be delivered to FNAL for attachment to the vacuum pipe. In year 3, FNAL will complete installation of the vacuum pipe. Commissioning of the assembled detector will begin immediately thereafter. The first science results will be demonstrations of macroscopic quantum superpositions (these demonstrations will occur during the commissioning phase). The interferometer is expected to be fully operational and taking physics data by the end of year 3 .

\begin{tabular}{|c|c|c|c|}
\hline Year & & Stanford & Fermilab \\
\hline 1 & $\begin{array}{l}\text { Q1 } \\
\text { Q2 } \\
\text { Q3 } \\
\text { Q4 }\end{array}$ & $\begin{array}{l}\text { Complete prelim. engineering } \\
\text { Atom sources \& lasers procured } \\
\text { Vac. vessel \& shielding procured }\end{array}$ & $\begin{array}{l}\text { Complete prelim. engineering } \\
\text { Complete vac. vessel design }\end{array}$ \\
\hline 2 & $\begin{array}{l}\text { Q1 } \\
\text { Q2 } \\
\text { Q3 } \\
\text { Q4 }\end{array}$ & $\begin{array}{l}\text { Delivery of interferometer vac. vessel } \\
\text { Atom sources \& lasers delivered }\end{array}$ & $\begin{array}{c}\text { Complete site design } \\
\text { Vac. system pre-assembly complete }\end{array}$ \\
\hline 3 & $\begin{array}{l}\text { Q1 } \\
\text { Q2 } \\
\text { Q3 } \\
\text { Q4 }\end{array}$ & & $\begin{array}{c}\text { Site outfitting complete. } \\
\text { Begin commissioning. First quantum physics results } \\
\text { First Dark Matter results }\end{array}$ \\
\hline
\end{tabular}

Table 8: Major MAGIS-100 milestones in an illustrative technically driven schedule. 


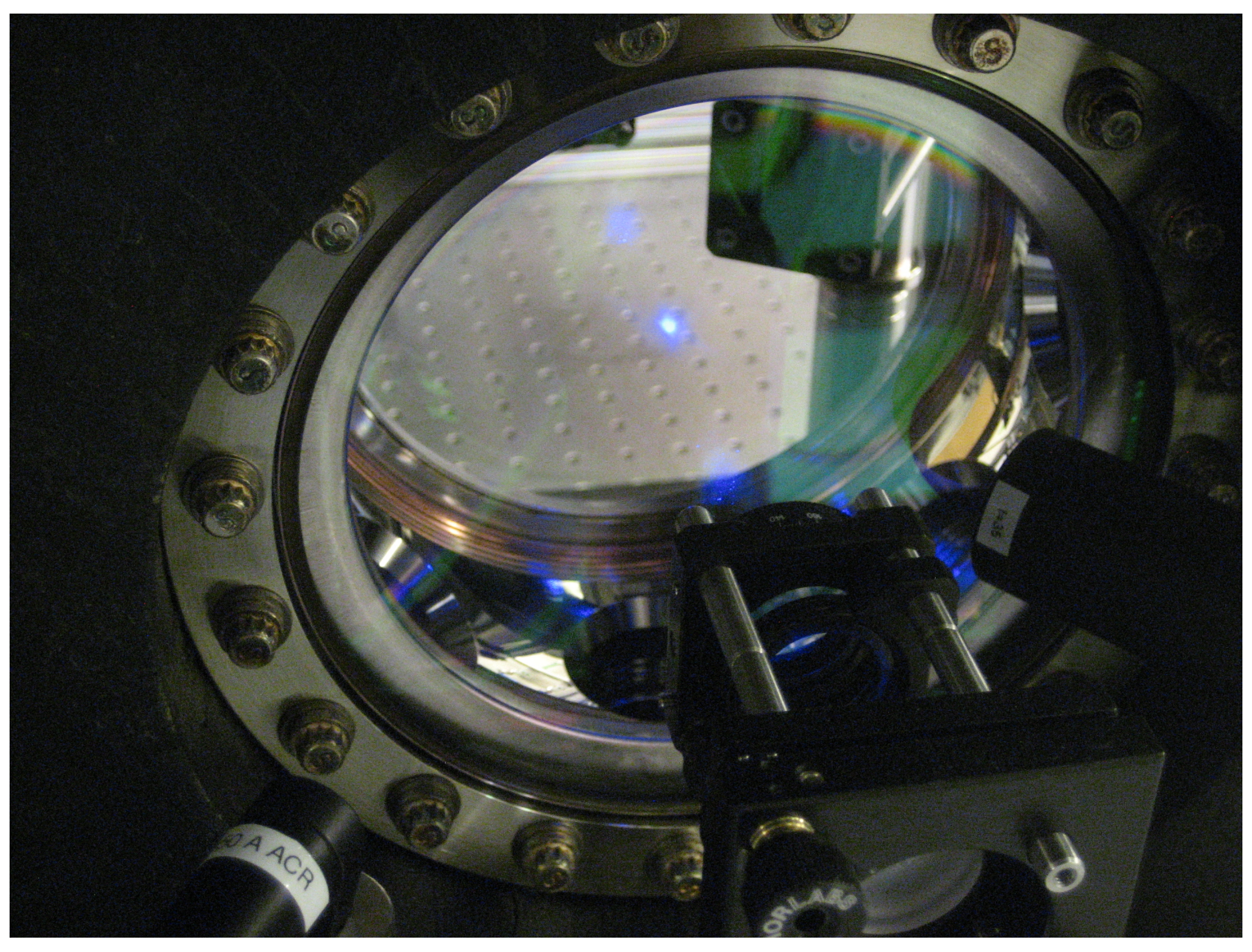

Figure 21: Cold Strontium atom cloud imaged in the R\&D setup at Stanford.

\begin{tabular}{|l|c|c|c|c|}
\hline Science Topic & $\begin{array}{c}\text { Required number } \\
\text { of atoms }\end{array}$ & $\begin{array}{c}\text { Atoms/sec when } \\
\text { taking science data }\end{array}$ & $\begin{array}{c}\text { Fraction of calendar } \\
\text { taking science data }\end{array}$ & $\begin{array}{c}\text { Estimated run } \\
\text { time (years) }\end{array}$ \\
\hline Commissioning & N/A & N/A & N/A & 1 \\
Phase 1: Quantum Science & $3 \times 10^{12}$ & $10^{6}$ & 0.1 & 0.5 \\
Phase 2: Dark Sector Campaign & $10^{15}$ & $10^{8}$ & 0.3 & 1 \\
Phase 3: Mid-band development & $10^{15}$ & $10^{8}$ & 0.3 & 1 \\
\hline
\end{tabular}

Table 9: Illustrative run plan. The science program is organized in three phases. Listed are preliminary estimates of (i) the number of atoms that must be launched/dropped to accomplish the required statistical precision for each phase assuming shot noise limited phase resolution (see figures in physics section), (ii) the average rate at which these atoms can be launched/dropped during science data taking, (iii) the fraction of calendar time the experiment is taking science data c.f. setup, calibrations etc., hence (iv) the calendar time.

The proposed subsequent science program would proceed in the following phases:

- Phase 1: Commissioning and Quantum Initiative. Demonstration of large wavepacket separation and long duration interferometry. Quantum superposition over macroscopic distances and times.

- Phase 2: First dark sector search campaign. Long baseline configuration at initial sensitivity. 
Search for ultralight scalar dark matter, new forces.

- Phase 3: Gravitational wave detector development. Demonstrations of detector enhancements such as resonant interferometry. Investigation of GGN suppression.

Preliminary estimates of the number of launched/dropped atoms and the required running time are shown, for each phase, in Table 9.

\subsection{Towards a Risk Register}

MAGIS-100 will maintain a list of risks that can impact the timetable of the detector project. The currently identified leading risks are:

- Atom source delayed due to cost or technology.

Probability: Low ; Impact: High

Mitigation: Where possible, atom source will be based on minimum modification of existing, proven designs.

- Shaft vibration, water ingress, or magnetic fields more difficult than anticipated.

Probability: Low; Impact: Medium

Mitigation: Ensure robust engineering requirements for support structure and/or magnetic shield.

- Appropriate experimental chamber vacuum levels more difficult to achieve than expected.

Probability: Low ; Impact: Medium

Mitigation: Use first vacuum chamber sections to test design.

Finally, we note that, due to the nature of the technology, there is some uncertainty in the estimated time needed to commission the experiment. Although we believe that 6 months is a reasonable estimate for this if all goes smoothly, it is possible that up to 12 months might be needed to get everything working sufficiently well for the first science measurements.

\section{Interactions with Funding Agencies}

In April 2018 a Letter of Intent was submitted in response to the DOE-OHEP "Quantum Information Science Enabled Discovery for High Energy Physics" Funding Opportunity Announcement (FOA). To fit within the scope of the FOA, the MAGIS-100 request $(\$ 3,988 \mathrm{~K})$ was designed to facilitate the launch of the MAGIS-100 initiative with a pilot setup that uses a single atom source. This pilot program would enable an initial exciting science program. The support of DOE-OHEP is essential for MAGIS-100 since the Collaboration proposes to locate the experiment at Fermilab. It was noted at the time of this QIS LOI that the Collaboration was also seeking support from other sources to complement the requested DOE support, and enable the full three-atom-source MAGIS-100 setup and science program.

In June 2018 the MAGIS-100 Collaboration submitted a proposal to the Gordon and Betty Moore Foundation (GBMF). It was intended that the requested support $(\$ 9,817 \mathrm{~K})$ would complement funding from the DOE and enable the full MAGIS-100 setup and science program. The proposal included a research and development component to enhance atom interferometer sensitivity to the level needed for future gravitational wave detection. In November 2018 GBMF awarded the Collaboration with a grant of $\$ 9,817 \mathrm{~K}$ for this work. The award is for five years, with a start date of January 2019. 
The DOE-OHEP 2018 request was considered to be beyond the scope of the FOA, and was therefore not funded. Based on further interactions with DOE-OHEP, together with the successful outcome of the Moore Foundation request, the MAGIS-100 Collaboration plans to respond to the next appropriate DOE-OHEP FOA with a request for support to complement the Moore Foundation grant and enable the full MAGIS-100 experiment described in this proposal. We anticipate this FOA will be in FY 2019.

Finally, the Collaboration is also seeking other support, including international support, for example from the UK. It is intended that, with this extra support, the Collaboration will be able to make an initial study of how to implement MAGIS-1000 (a $1 \mathrm{~km}$ scale setup beyond the MAGIS100 experiment), possibly in an existing shaft at SURF, and pursue an enhanced level of R\&D towards MAGIS-1000. These activities would also inform that part of the MAGIS-100 program that can be considered R\&D for MAGIS-1000. 


\section{References}

[1] Peter Asenbaum, Chris Overstreet, Tim Kovachy, Daniel D. Brown, Jason M. Hogan, and Mark A. Kasevich. Phase shift in an atom interferometer due to spacetime curvature across its wave function. Phys. Rev. Lett., 118:183602, May 2017.

[2] Swapan Chattopadhyay, David DeMille, Peter Graham, Roger Falcone, Evelyn Hu, Misha Lukin, Mark Kasevich, Nergis Mavalvala, Chris Monroe, Holger Mueller, Surjeet Rajendran, Cindy Regal, Mike Romalis, David Schuster, Alex Sushkov, Irfan Siddiqui, Kartik Srinivasan, Chris Stubbs, Ronald Walsworth, and Jun Ye. Quantum sensors at the intersections of fundamental science, quantum information science and computing. DoE Community Report, 2016.

[3] E. Farhi, S. Jordan, P. Hayden, M. Lukin, J. Maldacena, J. Preskill, P. Shor, J. Taylor, and C. Williams. Grand challenges at the interface of quantum information science, particle physics, and computing. DoE Community Report, 2015.

[4] Marco Battaglieri, Alberto Belloni, Aaron Chou, Priscilla Cushman, Bertrand Echenard, Rouven Essig, Juan Estrada, Jonathan L Feng, Brenna Flaugher, Patrick J Fox, et al. Us cosmic visions: New ideas in dark matter 2017: Community report. arXiv preprint arXiv:1707.04591, 2017.

[5] Steve Ritz et. al. Building for discovery: Strategic plan for u.s. particle physics in the global context. DoE Community Report, 2016.

[6] Asimina Arvanitaki, Peter W Graham, Jason M Hogan, Surjeet Rajendran, and Ken Van Tilburg. Search for light scalar dark matter with atomic gravitational wave detectors. arXiv preprint arXiv:1606.04541, 2016.

[7] Peter W. Graham, David E. Kaplan, Jeremy Mardon, Surjeet Rajendran, William A. Terrano, Lutz Trahms, and Thomas Wilkason. Spin Precession Experiments for Light Axionic Dark Matter. 2017.

[8] Peter W. Graham, David E. Kaplan, Jeremy Mardon, Surjeet Rajendran, and William A. Terrano. Dark Matter Direct Detection with Accelerometers. Phys. Rev., D93(7):075029, 2016.

[9] M. G. Tarallo, T. Mazzoni, N. Poli, D. V. Sutyrin, X. Zhang, and G. M. Tino. Test of einstein equivalence principle for 0-spin and half-integer-spin atoms: Search for spin-gravity coupling effects. Phys. Rev. Lett., 113:023005, Jul 2014.

[10] A. Bonnin, N. Zahzam, Y. Bidel, and A. Bresson. Simultaneous dual-species matter-wave accelerometer. Phys. Rev. A, 88:043615, Oct 2013.

[11] C. C. N. Kuhn, G. D. McDonald, K. S. Hardman, S. Bennetts, P. J. Everitt, P. A. Altin, J. E. Debs, J. D. Close, and N. P. Robins. A bose-condensed, simultaneous dual-species mach-zehnder atom interferometer. New Journal of Physics, 16(7):073035, 2014.

[12] Chris Overstreet, Peter Asenbaum, Tim Kovachy, Remy Notermans, Jason M Hogan, and Mark A Kasevich. Effective inertial frame in an atom interferometric test of the equivalence principle. Physical review letters, 120(18):183604, 2018.

[13] J. E. Moody and Frank Wilczek. NEW MACROSCOPIC FORCES? Phys. Rev., D30:130, 1984 . 
[14] Peter W. Graham, David E. Kaplan, and Surjeet Rajendran. Cosmological Relaxation of the Electroweak Scale. Phys. Rev. Lett., 115(22):221801, 2015.

[15] Nima Arkani-Hamed, Savas Dimopoulos, and G. R. Dvali. Phenomenology, astrophysics and cosmology of theories with submillimeter dimensions and TeV scale quantum gravity. Phys. Rev., D59:086004, 1999.

[16] S. Dimopoulos and G. F. Giudice. Macroscopic forces from supersymmetry. Phys. Lett., B379:105-114, 1996.

[17] T Kovachy, P Asenbaum, C Overstreet, CA Donnelly, SM Dickerson, A Sugarbaker, JM Hogan, and MA Kasevich. Quantum superposition at the half-metre scale. Nature, 528(7583):530-533, 2015.

[18] Onur Hosten, Nils J Engelsen, Rajiv Krishnakumar, and Mark A Kasevich. Measurement noise 100 times lower than the quantum-projection limit using entangled atoms. Nature, 529(7587):505-508, 2016.

[19] Justin G Bohnet, Kevin C Cox, Matthew A Norcia, Joshua M Weiner, Zilong Chen, and James K Thompson. Reduced spin measurement back-action for a phase sensitivity ten times beyond the standard quantum limit. Nature Photonics, 8(9):731-736, 2014.

[20] Ayaka Shoda, Yuya Kuwahara, Masaki Ando, Kazunari Eda, Kodai Tejima, Yoichi Aso, and Yousuke Itoh. Ground-based low-frequency gravitational-wave detector with multiple outputs. Phys. Rev. D, 95:082004, Apr 2017.

[21] Savas Dimopoulos, Peter W. Graham, Jason M. Hogan, Mark A. Kasevich, and Surjeet Rajendran. Atomic gravitational wave interferometric sensor. Physical Review D, 78(12):122002, 2008 .

[22] Jason M. Hogan, David M. S. Johnson, Susannah Dickerson, Tim Kovachy, Alex Sugarbaker, Sheng-wey Chiow, Peter W. Graham, Mark A. Kasevich, Babak Saif, Surjeet Rajendran, et al. An atomic gravitational wave interferometric sensor in low earth orbit (agis-leo). General Relativity and Gravitation, 43(7):1953-2009, 2011.

[23] Peter W Graham, Jason M Hogan, Mark A Kasevich, and Surjeet Rajendran. New method for gravitational wave detection with atomic sensors. Physical review letters, 110(17):171102, 2013.

[24] B. Canuel, A. Bertoldi, L. Amand, E. Borgo di Pozzo, B. Fang, R. Geiger, J. Gillot, S. Henry, J. Hinderer, D. Holleville, G. Lefèvre, M. Merzougui, N. Mielec, T. Monfret, S. Pelisson, M. Prevedelli, S. Reynaud, I. Riou, Y. Rogister, S. Rosat, E. Cormier, A. Landragin, W. Chaibi, S. Gaffet, and P. Bouyer. Exploring gravity with the MIGA large scale atom interferometer. ArXiv e-prints, March 2017.

[25] Peter W. Graham, Jason M. Hogan, Mark A. Kasevich, and Surjeet Rajendran. Resonant mode for gravitational wave detectors based on atom interferometry. Phys. Rev., D94(10):104022, 2016.

[26] S. Kolkowitz, I. Pikovski, N. Langellier, M. D. Lukin, R. L. Walsworth, and J. Ye. Gravitational wave detection with optical lattice atomic clocks. Phys. Rev. D, 94:124043, Dec 2016. 
[27] Matthew A. Norcia, Julia R. K. Cline, and James K. Thompson. Role of atoms in atomic gravitational-wave detectors. Phys. Rev. A, 96:042118, Oct 2017.

[28] Peter W Graham, Jason M Hogan, Mark A Kasevich, Surjeet Rajendran, and Roger W Romani. Mid-band gravitational wave detection with precision atomic sensors. arXiv preprint arXiv:1711.02225, 2017.

[29] Savas Dimopoulos, Peter W. Graham, Jason M. Hogan, and Mark A. Kasevich. General relativistic effects in atom interferometry. Phys. Rev. D, 78:042003, Aug 2008.

[30] Nan Yu and Massimo Tinto. Gravitational wave detection with single-laser atom interferometers. General Relativity and Gravitation, 43(7):1943-1952, Jul 2011.

[31] Sara L. Campbell, R. B. Hutson, G. E. Marti, A. Goban, N. Darkwah Oppong, R. L. McNally, L. Sonderhouse, J. M. Robinson, W. Zhang, B. J. Bloom, et al. A fermi-degenerate threedimensional optical lattice clock. Science, 358(6359):90-94, 2017.

[32] Marco Schioppo, Roger C. Brown, William F. McGrew, N. Hinkley, Robert J. Fasano, K. Beloy, T. H. Yoon, Gianmaria Milani, D. Nicolodi, J. A. Sherman, et al. Ultrastable optical clock with two cold-atom ensembles. Nature Photonics, 11(1):48, 2017.

[33] Peter W. Graham and Sunghoon Jung. Localizing Gravitational Wave Sources with SingleBaseline Atom Interferometers. 2017.

[34] Ilya Mandel, Alberto Sesana, and Alberto Vecchio. The astrophysical science case for a decihertz gravitational-wave detector. 2017.

[35] Asimina Arvanitaki, Savas Dimopoulos, Sergei Dubovsky, Nemanja Kaloper, and John MarchRussell. String Axiverse. Phys. Rev., D81:123530, 2010.

[36] Bernard F. Schutz. Determining the Hubble Constant from Gravitational Wave Observations. Nature, 323:310-311, 1986.

[37] Hsin-Yu Chen and Daniel E. Holz. Finding the One: Identifying the Host Galaxies of Gravitational-Wave Sources. 2016.

[38] Jeffrey Michael Mcguirk, GT Foster, JB Fixler, MJ Snadden, and MA Kasevich. Sensitive absolute-gravity gradiometry using atom interferometry. Physical Review A, 65(3):033608, 2002 .

[39] TL Nicholson, SL Campbell, RB Hutson, GE Marti, BJ Bloom, RL McNally, Wei Zhang, MD Barrett, MS Safronova, GF Strouse, et al. Systematic evaluation of an atomic clock at $2 \times 10$ - 18 total uncertainty. Nature communications, 6:6896, 2015.

[40] Mark Kasevich and Steven Chu. Atomic interferometry using stimulated raman transitions. Physical review letters, 67(2):181, 1991.

[41] AV Taichenachev, VI Yudin, CW Oates, CW Hoyt, ZW Barber, and L Hollberg. Magnetic field-induced spectroscopy of forbidden optical transitions with application to lattice-based optical atomic clocks. Physical review letters, 96(8):083001, 2006.

[42] W. Chaibi, R. Geiger, B. Canuel, A. Bertoldi, A. Landragin, and P. Bouyer. Low frequency gravitational wave detection with ground-based atom interferometer arrays. Phys. Rev. D, 93:021101, Jan 2016. 
[43] Mark Kasevich and Steven Chu. Atomic interferometry using stimulated raman transitions. Phys. Rev. Lett., 67:181-184, Jul 1991.

[44] G. M. Tino and M. A. Kasevich. Atom interferometry. Proceedings of the International School of Physics" Enrico Fermi", Course CLXXXVIII, Varenna, 2013.

[45] TL Gustavson, P Bouyer, and MA Kasevich. Precision rotation measurements with an atom interferometer gyroscope. Physical Review Letters, 78(11):2046, 1997.

[46] Achim Peters, Keng Yeow Chung, and Steven Chu. Measurement of gravitational acceleration by dropping atoms. Nature, 400(6747):849-852, 1999.

[47] Jeffrey B Fixler, GT Foster, JM McGuirk, and MA Kasevich. Atom interferometer measurement of the newtonian constant of gravity. Science, 315(5808):74-77, 2007.

[48] G. Lamporesi, A. Bertoldi, L. Cacciapuoti, M. Prevedelli, and G. M. Tino. Determination of the newtonian gravitational constant using atom interferometry. Phys. Rev. Lett., 100:050801, Feb 2008.

[49] G. Rosi, F. Sorrentino, L. Cacciapuoti, M. Prevedelli, and G. M. Tino. Precision measurement of the newtonian gravitational constant using cold atoms. Nature, 510(7506):518-521, 2014.

[50] Malo Cadoret, Estefania de Mirandes, Pierre Cladé, Saïda Guellati-Khélifa, Catherine Schwob, Fran çois Nez, Lucile Julien, and Fran çois Biraben. Combination of bloch oscillations with a ramsey-bordé interferometer: New determination of the fine structure constant. Phys. Rev. Lett., 101:230801, Dec 2008.

[51] R. Bouchendira, P. Cladé, S. Guellati-Khélifa, F. Nez, and F. Biraben. New Determination of the Fine Structure Constant and Test of the Quantum Electrodynamics. Phys. Rev. Lett., 106(8):080801, February 2011.

[52] Richard H. Parker, Chenghui Yu, Weicheng Zhong, Brian Estey, and Holger Müller. Measurement of the fine-structure constant as a test of the standard model. Science, 360(6385):191-195, 2018.

[53] Tim Kovachy, Jason M Hogan, Alex Sugarbaker, Susannah M Dickerson, Christine A Donnelly, Chris Overstreet, and Mark A Kasevich. Matter wave lensing to picokelvin temperatures. Physical review letters, 114(14):143004, 2015.

[54] Susannah M. Dickerson, Jason M. Hogan, Alex Sugarbaker, David M. S. Johnson, and Mark A. Kasevich. Multiaxis inertial sensing with long-time point source atom interferometry. Phys. Rev. Lett., 111:083001, Aug 2013.

[55] Sheng-wey Chiow, Tim Kovachy, Hui-Chun Chien, and Mark A Kasevich. 102 hk large area atom interferometers. Physical review letters, 107(13):130403, 2011.

[56] Holger Müller, Sheng-wey Chiow, Quan Long, Sven Herrmann, and Steven Chu. Atom interferometry with up to 24-photon-momentum-transfer beam splitters. Phys. Rev. Lett., 100:180405, May 2008.

[57] A Peters, K Y Chung, and S Chu. High-precision gravity measurements using atom interferometry. Metrologia, 38(1):25, 2001. 
[58] Jason M. Hogan, David M. S. Johnson, and Mark A. Kasevich. Light-pulse atom interferometry. In E. Arimondo, W. Ertmer, and W. P. Schleich, editors, Proc. Int. Sch. Phys. "Enrico Fermi" Atom Opt. Sp. Phys., pages 411-447, Amsterdam, June 2009. IOS Press.

[59] C Antoine and C J Bordé. Quantum theory of atomic clocks and gravito-inertial sensors: an update. J. Opt. B Quantum Semiclassical Opt., 5(2):S199, 2003.

[60] Susannah M. Dickerson, Jason M. Hogan, Alex Sugarbaker, David M. S. Johnson, and Mark A. Kasevich. Multiaxis inertial sensing with long-time point source atom interferometry. Phys. Rev. Lett., 111:083001, Aug 2013.

[61] A. Sugarbaker, S. M. Dickerson, J. M. Hogan, D. M. S. Johnson, and M. A. Kasevich. Enhanced Atom Interferometer Readout through the Application of Phase Shear. Phys. Rev. Lett., 111(11):113002, September 2013.

[62] Sheng-wey Chiow, Tim Kovachy, Jason M. Hogan, and Mark A. Kasevich. Generation of 43 $\mathrm{w}$ of quasi-continuous $780 \mathrm{~nm}$ laser light via high-efficiency, single-pass frequency doubling in periodically poled lithium niobate crystals. Optics letters, 37(18):3861-3863, 2012.

[63] Susannah Dickerson, Jason M. Hogan, David M. S. Johnson, Tim Kovachy, Alex Sugarbaker, Sheng-wey Chiow, and Mark A. Kasevich. A high-performance magnetic shield with large length-to-diameter ratio. Review of Scientific Instruments, 83(6):065108, 2012.

[64] St. Falke, H. Schnatz, J. S. R. Vellore Winfred, Th. Middelmann, St. Vogt, S. Weyers, B. Lipphardt, G. Grosche, F. Riehle, U. Sterr, et al. The $87 \mathrm{sr}$ optical frequency standard at ptb. Metrologia, 48(5):399, 2011.

[65] R. Corgier, S. Amri, W. Herr, H. Ahlers, J. Rudolph, D. Guéry-Odelin, E. M. Rasel, E. Charron, and N. Gaaloul. Fast manipulation of bose-einstein condensates with an atom chip. New Journal of Physics, 20(5):055002, 2018.

[66] Harold J. Metcalf and Peter Van der Straten. Laser cooling and trapping of neutral atoms. Wiley Online Library, 2007.

[67] Shau-Yu Lan, Pei-Chen Kuan, Brian Estey, Philipp Haslinger, and Holger Müller. Influence of the coriolis force in atom interferometry. Phys. Rev. Lett., 108:090402, Feb 2012.

[68] B. Dubetsky and M. A. Kasevich. Atom interferometer as a selective sensor of rotation or gravity. Physical Review A, 74(2):023615, 2006.

[69] Peter W. Graham, Jason M. Hogan, Mark A. Kasevich, Surjeet Rajendran, and Roger W. Romani. Mid-band gravitational wave detection with precision atomic sensors. 2017.

[70] J. M. Hogan, J. Hammer, S.-w. Chiow, S. Dickerson, D. M. S. Johnson, T. Kovachy, A. Sugarbaker, and M. A. Kasevich. Precision angle sensor using an optical lever inside a sagnac interferometer. Optics letters, 36(9):1698-1700, 2011.

[71] Matthew D. Swallows, Michael J. Martin, Michael Bishof, Craig Benko, Yige Lin, Sebastian Blatt, Ana Maria Rey, and Jun Ye. Operating a 87 sr optical lattice clock with high precision and at high density. IEEE transactions on ultrasonics, ferroelectrics, and frequency control, $59(3): 416-425,2012$. 
[72] Malcolm H Levitt. Symmetrical composite pulse sequences for nmr population inversion. i. compensation of radiofrequency field inhomogeneity. Journal of Magnetic Resonance (1969), 48(2):234 - 264, 1982.

[73] Shayne Bennetts, Chun-Chia Chen, Benjamin Pasquiou, Florian Schreck, et al. Steady-state magneto-optical trap with 100-fold improved phase-space density. Physical review letters, 119(22):223202, 2017.

[74] Kevin C. Cox, Graham P. Greve, Joshua M. Weiner, and James K. Thompson. Deterministic squeezed states with collective measurements and feedback. Phys. Rev. Lett., 116:093602, Mar 2016.

[75] Liang Hu, Nicola Poli, Leonardo Salvi, and Guglielmo M. Tino. Atom interferometry with the sr optical clock transition. Physical review letters, 119(26):263601, 2017.

[76] Martin M. Boyd. High precision spectroscopy of strontium in an optical lattice: Towards a new standard for frequency and time. PhD thesis, University of Colorado at Boulder, 2007.

[77] Kakeru Takahashi, Masaki Ando, and Kimio Tsubono. Stabilization of laser intensity and frequency using optical fiber. In Journal of Physics: Conference Series, volume 122, page 012016. IOP Publishing, 2008.

[78] Albert Roura. Circumventing heisenberg's uncertainty principle in atom interferometry tests of the equivalence principle. Phys. Rev. Lett., 118:160401, Apr 2017.

[79] G. D'Amico, G. Rosi, S. Zhan, L. Cacciapuoti, M. Fattori, and G. M. Tino. Canceling the gravity gradient phase shift in atom interferometry. Phys. Rev. Lett., 119:253201, Dec 2017.

[80] H. Ahlers, H. Müntinga, A. Wenzlawski, M. Krutzik, G. Tackmann, S. Abend, N. Gaaloul, E. Giese, A. Roura, R. Kuhl, C. Lämmerzahl, A. Peters, P. Windpassinger, K. Sengstock, W. P. Schleich, W. Ertmer, and E. M. Rasel. Double Bragg Interferometry. Phys. Rev. Lett., 116(17):173601, 2016.

[81] Malcolm H. Levitt. Composite pulses. Wiley Online Library, 2007.

[82] B. P. Abbott et al. Ligo: the laser interferometer gravitational-wave observatory. Reports on Progress in Physics, 72(7):076901, 2009.

[83] Jan Harms. Terrestrial Gravity Fluctuations. Living Rev. Rel., 18:3, 2015.

[84] A. E. Siegman. Lasers. University Science Books, 1986.

[85] A. Wicht, E. Sarajlic, J. M. Hensley, and S. Chu. Phase shifts in precision atom interferometry due to the localization of atoms and optical fields. Phys. Rev. A, 72:023602, Aug 2005. 


\section{A Appendix: Fermilab contributions}

In addition to hosting MAGIS-100, the laboratory brings significant scientific and technical capabilities to the project. Fermilab scientists have expertise in design and implementation of systems on the scale of hundreds of meters to kilometers. In particular, the requirements of outfitting a large accelerator or HEP experiment give experience in the technical skills of vacuum, magnetic fields and shielding, detector readout, and controls. In addition, Fermilab has experience of working in large collaborations with multiple groups, supervision of engineering and construction and project management of distributed efforts.

\section{A.1 Proposed Fermilab Contributions}

- Scientific Research Effort(Adamson, Geer, Harnik Plunkett).

- Building maintenance and services

- Remote Operations Center (ROC) maintenance and services.

- Support of sitewide computing network.

- Support of accelerator controls network.

- Safety and environmental support.

\section{A.2 Fermilab Facilities}

- ACNET accelerator controls infrastructure (present in MINOS building) for monitoring and logging of environmental data.

- Fully equipped Remote Operations Center (ROC) for detector operations.

- Vacuum diagnostic equipment (leak detectors, RGA system, roughing pumps).

- 3D Printers for production of non-magnetic parts.

- Equipment pool for general electronics.

- Tools and general mechanical equipment.

\section{B Appendix: Stanford contributions}

Scientific research effort at Stanford will be contributed by Hogan, Graham, and Kasevich. Hogan has experience with the design and construction of $10 \mathrm{~m}$ scale atom interferometers, and leads a group developing atom interferometry using clock atoms, the key technology of MAGIS-100. Graham proposed novel ways to use MAGIS to detect ultralight dark matter such as axions, and found important and novel gravitational wave science accessible in the mid-band such as precise angular localization of gravitational wave sources. Kasevich pioneered many of the light-pulse atom interferometry techniques that underlie the MAGIS-100 detector, and is PI for the Stanford $10 \mathrm{~m}$ atom interferometer where many of the proof-of-concept measurements for MAGIS-100 were performed. Stanford will take part in the following aspects of the proposed work. 


\section{B.1 Proposed Stanford Contributions}

- Scientific Research Effort: Hogan (0.5 FTE), Graham (0.2 FTE), Kasevich (0.2 FTE)

- Design, construction, and validation of the three atom sources (Hogan).

- Overall instrument design, commissioning, and operation (Hogan, Kasevich).

- Modeling of gravitational wave source sensitivity, sky localization (Graham, Hogan).

- Investigate strategies to search for axions and other ultralight dark matter, as well as black hole spin measurements (Graham).

- Data analysis (Graham, Hogan, Kasevich).

- Development of next-generation atom source using quantum entanglement for reduced readout noise (Kasevich).

- Development of next-generation atom source with higher atomic flux for reduced readout noise (Hogan).

\section{Appendix: Northern Illinois University contributions}

The Northern Illinois University effort will initially consist of 0.5 FTE of scientific effort by academic faculty co-PI Prof. Swapan Chattopadhyay to guide the project, and contribute the full-time 1 FTE effort, under his supervision, of a $\mathrm{PhD}$ graduate student (Jeremiah Mitchell) participating in the project in theoretical calculations, data acquisition and analysis. NIU will also contribute a stateof-the-art seismometer and associated calibration and recording accessories - such special-purpose hardware will be necessary towards environmental monitoring of terrestrial noise for eventual characterization of various components of the systematics such as 'gravity gradient noise', which are critical to the success of the experiment. The Northern Illinois University also plans to work in close collaboration with Northwestern University PI Tim Kovachy in setting up prototype R\&D table-top facilities for benchmarking laser wavefront aberration and jitter. The NIU contribution is expected to rise to $2 \mathrm{PhD}$ students, 1 post-doctoral fellow and $0.7 \mathrm{FTE}$ of senior scientific effort by the third year.

\section{C.1 Proposed NIU Contributions}

- Scientific Research and Supervision Effort (PI: Swapan Chattopadhyay)

- Procure/purchase, install, calibrate and operate state-of-the-art seismometer on site at Fermilab at the MAGIS-100 site.

- Obtain and analyze seismic data.

- Convert into ambient Gravity Gradient Noise spectra.

- Model laser wavefront aberration and its mitigation via collaborative studies at Northwestern test-stand.

- Coriolis motion modeling and mitigation.

- Error modeling to ensure maximum sensitivity for imaging and phase measurements.

- Contribute possible Vacuum parts and Equipment as needed.

- Support ongoing efforts at Fermilab as needed. 


\section{Appendix: Northwestern contributions}

The Northwestern contributions will be carried out by Kovachy's research group. Kovachy has expertise in large momentum transfer atom optics, ultra-sensitive macroscopic scale atom interferometry, simultaneous atom interferometers with different atomic species, atom interferometry error modeling, matter wave lensing to ultra-low effective temperatures, and high-power laser systems. Northwestern will take part in the following aspects of the proposed work.

\section{D.1 Proposed Northwestern Contributions}

- Scientific research effort: Kovachy (0.3 FTE).

- Design and construction of the atom interferometry laser system.

- Instrument design, commissioning, and operation.

- Implementation of noise mitigation strategies (detailed in Sec. 6 and Appendix H) to ensure that the instrument reaches its design performance.

- Data analysis.

\section{E Appendix: Berkeley contributions}

The Berkeley contributions will be carried out by Rajendran's research group. Rajendran helped propose and develop the MAGIS detector concept and has extensive experience with systematic error analysis for gravitational wave detection using atom interferometry. He has also developed innovative theories such as the relaxion framework to address fundamental problems in particle physics such as the hierarchy problem and identified how relaxion dark matter could be detected in experiments such as MAGIS. Berkeley will take part in the following aspects of the proposed work.

\section{E.1 Proposed Berkeley Contributions}

- Scientific research effort: Rajendran (0.2 FTE).

- Develop strategies to search for axions and other ultralight dark matter.

- Explore possibilities for novel gravitational wave science such as black hole spin measurements.

- Analyze and develop strategies to mitigate backgrounds such as gravity gradient noise.

- Data analysis and interpretation.

\section{F Appendix: University of Liverpool contributions}

The University of Liverpool contributions will be led by Coleman within the Particle Physics group, already funded via the groups STFC consolidated grant and the Royal Society of London. Furthermore, the group is bidding for capital and research funds for MAGIS (submission Jan 2019 to UKRI), and will be submitting proposals for Fellowships to the UK funding agencies. The group brings experience in both the design and construction of detectors for fundamental physics as well as atom interferometry. The Liverpool contribution will bring mechanical and electronic engineering design to the project, and work in conjunction with UK industry in Lasers photonics. Liverpool will take part in the following aspects of the proposed work. 


\section{F.1 Proposed Liverpool Contributions}

- Scientific research effort: Coleman (0.5 FTE) plus (0.5 FTE) engineering, plus additional core research effort (DAQ, computing) as required.

- The UK hardware contribution being requested is for: Interferometry lasers with M Squared Ltd; EMCCD cameras; support for the FNAL camera program; development towards the DAQ and control system.

- Developing an analysis and simulation framework for the experiment, including blinding techniques, data quality, and image processing.

- A substantial fraction of time is foreseen to be spent at Fermilab to contribute towards the construction, commissioning, as well as operations and data-taking. 


\section{G Appendix: MAGIS-1000, a long-term vision}

\begin{tabular}{|c|c|c|c|c|c|}
\hline Experiments & Proposed Site & Baseline $L$ & LMT Atom optics $N$ & Atom sources & Phase noise $\delta \phi$ \\
\hline Sr prototype & Stanford (Hogan lab) & $5 \mathrm{~m}$ & $10^{2}$ & 2 & $10^{-3} \mathrm{rad} / \sqrt{\mathrm{Hz}}$ \\
MAGIS-100 & FermiLab, NuMI shaft & $100 \mathrm{~m}$ & $10^{3}$ & 3 & $10^{-4} \mathrm{rad} / \sqrt{\mathrm{Hz}}$ \\
MAGIS-100 (5 year) & FermiLab, NuMI shaft & $100 \mathrm{~m}$ & $\approx 10^{4}$ & 3 & $10^{-5} \mathrm{rad} / \sqrt{\mathrm{Hz}}$ \\
Terrestrial & Homestake mine & $\approx 4 \mathrm{~km}$ & $10^{3}$ & 10 & $10^{-5} \mathrm{rad} / \sqrt{\mathrm{Hz}}$ \\
Satellite & Medium Earth orbit (MEO) & $4 \times 10^{7} \mathrm{~m}$ & $10^{3}$ & 2 & $10^{-4} \mathrm{rad} / \sqrt{\mathrm{Hz}}$ \\
\hline
\end{tabular}

Table 10: A long-term vision of the MAGIS Collaboration technology development and associated experimental program. The listed numbers for MAGIS-100 correspond to the initial R\&D effort plus further development to push LMT atom optics to their full physically allowable extent.

MAGIS-100 can be thought of as the next step in a multi-step program which starts with the existing 10m-scale experiment described in Section 4, and progresses through MAGIS-100 to the ultimate terrestrial-based experiment with a $1 \mathrm{~km}$ scale baseline (MAGIS-1000), and perhaps eventually to an experiment in space with an even longer baseline. A summary of the desired experimental parameters is given in Table 10.

The MAGIS-100 5-year program is designed to make significant progress toward the requirements of the $\mathrm{km}$-scale detector. The R\&D program will advance the detector technology to a level such that the scientifically interesting range of $10^{-20} / \sqrt{\mathrm{Hz}}$ can be reached by straightforward scaling of the baseline alone. The sensitivity of MAGIS-100 at the end of 5 years is at the $10^{-19} / \sqrt{\mathrm{Hz}}$ level. This level of sensitivity follows from implementing the R\&D program outlined in the proposal and using LMT atom optics to their full extent (consistent with known constraints). From there, we expect that simply translating to the longer baseline in the kilometer-scale instrument will yield a factor of 30 increase in sensitivity, as explained in the following. First, continuous vertical mine shafts of $2 \mathrm{~km}$ (or possibly longer) seem to be available at multiple potential sites, so a $2 \mathrm{~km}$ baseline appears feasible. This results in a factor of 20 increase. In addition, there will be a larger number of atom sources in the km-scale instrument (for gravity gradient compensation), and data from these can be averaged together to reduce noise. Assuming a similar atom source spacing as MAGIS-100, the scaled-up detector will have $\sim 10$ times the atom sources, yielding an improvement of $\sqrt{10} / 2 \sim 1.5$ (the factor of two reduction arises because the effective baseline for the additional atom sources is smaller on average). Together these factors suggest a sensitivity improvement of $20 \times 1.5=30$ just due to increasing the baseline. This already puts the detector well within the scientifically interesting zone $\left(<10^{-20} / \sqrt{\mathrm{Hz}}\right)$, where there are gravitational wave sources that have already been detected. Even if MAGIS-100 were to miss the target sensitivity by factor of 3, the kilometer-scale instrument would still be within the scientifically interesting range.

We argue that a kilometer-scale instrument can reach $10^{-21} / \sqrt{\mathrm{Hz}}$, another factor of 3 beyond the level that is reached by increasing the baseline. Achieving this last factor of 3 would require some additional R\&D work. This can be achieved through additional incremental improvements in atom flux and spin squeezing. After the 5-year program, we expect that it will be clearer which of these two is more promising. However, improvements in these areas are not incompatible, so the target could be reached through a combination of smaller improvements. We note that the fundamental limits are far away for both of these aspects of the sensor technology. The work needed is primarily of a technical nature, not conceptual. For example, the commercial $\mathrm{Sr}$ atom beam sources that we are currently using have a thermal flux of $10^{14}$ atoms/s, so only a small fraction needs to be harvested (and cooled) to reach $10^{10}$ cold atoms/s, the level needed for $10^{-21} / \sqrt{\mathrm{Hz}}$ strain sensitivity. An obvious bottleneck for realizing this at present is the large amount of laser power required on 
the cooling transition. We anticipate that this can be circumvented by straightforward investment in more powerful lasers.

A potential site for MAGIS-1000 is at the SURF Laboratory in South Dakota. The MAGIS-100 Collaboration will seek additional funding to make an initial study of MAGIS-1000 at SURF, both to better understand the challenges that must be met and to assess the suitability of the SURF shaft. This study would also inform that part of the MAGIS-100 program aimed at preparing for MAGIS-1000. 


\section{H Appendix: Systematics and Error Modeling}

The following describes the contributions to the error budget of the MAGIS-100 experiment and some of the strategies used in the detector design to minimize their impact. Modeling and analysis of many of the error contributions has been carried out in the context of atomic gravitational wave detectors-with direct applicability MAGIS-100 [21-23,69]. The MAGIS-100 experiment is designed to be sensitive to time-varying gravitational wave or dark matter signals in the frequency band $\sim 0.1-10 \mathrm{~Hz}$. The relevant backgrounds are those that temporally vary in this frequency band. This frequency selectivity eases a number of requirements. For instance, any sources of error that lead to constant phase offsets or long-term phase drifts would not affect the potential science signal. The initial version of MAGIS-100 (see the MAGIS-100 Initial curve in Fig. 4), which aims for 100 $k$ beam splitters and a phase resolution of $10^{-3} \mathrm{rad} / \sqrt{\mathrm{Hz}}$, appears achievable in the near term. The ultimate sensitivities require further research and development to boost instrument sensitivity and correspondingly reduce the influence of noise sources to the level shown in the MAGIS-100 (5 year) curve in Fig. 4, which assumes $4 \times 10^{4} \hbar k$ beam splitters, a pulse spacing on the order of $1 \mathrm{~s}$, and a phase resolution of $10^{-5} \mathrm{rad} / \sqrt{\mathrm{Hz}}$.

- Laser frequency noise. The atom-laser interactions are dependent of the phase of the laser at each of the interaction points, so laser technical noise can cause noise in the atom interferometer signal. The multiple atom ensembles in the gradiometer are subject to the same laser pulses, so this noise effect is expected to be common-mode suppressed to a significant degree. Single photon atom optics on the clock transition [75] will be employed to realize the necessary level of laser noise rejection, as detailed in Ref. [23]. Specifically, the residual noise $\delta \phi_{\text {freq }}$ in the interferometer phase arising from laser frequency noise has the leading contribution [23] $\delta \phi_{\text {freq }} \sim\left(10^{-14} \mathrm{rad} / \sqrt{\mathrm{Hz}}\right)\left(\frac{n}{100}\right)\left(\frac{\Delta v}{10 \mu \mathrm{m} / \mathrm{s}}\right)\left(\frac{\delta f}{10 \mathrm{~Hz} / \sqrt{\mathrm{Hz}}}\right)\left(\frac{\Delta \tau}{100 \mu \mathrm{s}}\right)$ related to the finite duration $\Delta \tau$ of each laser pulse, the velocity difference $\Delta v$ between the two atom clouds in the gradiometer, the beam splitter momentum $n \hbar k$, and the amplitude spectral density $\delta f$ of laser frequency noise. By using an optimized lensing sequence and a common velocity selection pulse for the two atom clouds, $\Delta v$ can be kept below $10 \mu \mathrm{m} / \mathrm{s}$. For all conceivable experimental parameters, $\delta \phi_{\text {freq }}$ is negligibly small.

- Laser wavefront aberrations. The influence of laser wavefront aberrations and associated mitigation strategies, including in situ wavefront measurements and spatial filtering of the atom optics laser beam via free propagation, are discussed in Section 6. Detailed modeling of the effect of wavefront aberrations on the phase of an atom interferometer was carried out in Ref. [22]. This interferometer phase response analysis can be combined with in situ wavefront measurements and measurements of atom cloud kinematics in MAGIS-100 to correct for backgrounds arising from wavefront aberrations. Recent work carried out by the MAGIS collaboration (to be submitted for publication, see Appendix $\mathrm{J}$ ) has expanded upon the treatment in [22] with a full perturbative Fourier analysis. This new work goes beyond the previous work to also include effective momentum kicks that arise from the wavefront aberration phase varying spatially in the transverse plane. The calculation is taken to second order in the aberration amplitude $\delta$ (in radians), and gives the same result at first order as previous calculations, as expected. As a quantitative example, the phase shift size of the leading order term and second order term in $\delta$ at different scales of large momentum transfer (LMT) will be considered. This example considers a laser with wavelength $\lambda=698 \mathrm{~nm}$ $(k=2 \pi / \lambda)$; interferometer height $H$; duration between the interferometer beam splitter and mirror $T$; and a transverse atom trajectory fluctuation of size $\Delta x$ during the interferometer, 
which could result from shot-to-shot variations in initial atom positions and/or velocities. To first order in $\delta$ :

$$
\begin{aligned}
\delta \phi_{w f, 1} & \sim n \delta k_{t} \Delta x \sin \left[\frac{H k_{t}^{2}}{2 k}\right] \\
& \sim\left(5 \times 10^{-5} \mathrm{rad} / \sqrt{\mathrm{Hz}}\right)\left(\frac{\delta}{0.005}\right)\left(\frac{n}{100}\right)\left(\frac{k_{t}}{(3 \mathrm{~mm})^{-1}}\right)\left(\frac{\Delta x}{1 \mu \mathrm{m} / \sqrt{\mathrm{Hz}}}\right)\left(\frac{\sin \left[H k_{t}^{2} / 2 k\right]}{0.3}\right) .
\end{aligned}
$$

For second order in $\delta$, the leading contribution is

$$
\begin{aligned}
\delta \phi_{w f, 2} & \sim \delta^{2} \frac{k_{t}^{2} n^{2} T \hbar}{m} k_{t} \Delta x \sin \left[\frac{H k_{t}^{2}}{2 k}\right] \\
& \sim\left(3 \times 10^{-9} \mathrm{rad} / \sqrt{\mathrm{Hz}}\right)\left(\frac{\delta}{0.005}\right)^{2}\left(\frac{n}{100}\right)^{2}\left(\frac{k_{t}}{(3 \mathrm{~mm})^{-1}}\right)^{3}\left(\frac{\Delta x}{1 \mu \mathrm{m} / \sqrt{\mathrm{Hz}}}\right)\left(\frac{\sin \left[H k_{t}^{2} / 2 k\right]}{0.3}\right),
\end{aligned}
$$

assuming $T=1 \mathrm{~s}$. The $\left(n k_{t}\right)^{2}$ dependence at second order comes from the kinetic energy of the momentum kicks from the transverse component of the local wave vector of the perturbed laser field. The additional $k_{t} \Delta x$ factor in both terms reflects the rate at which the phase shift varies with $\Delta x$, which increases proportionally with $k_{t}$. As discussed in Section 6 , wavefront perturbations with a spatial scale of $\lesssim 1 \mathrm{~mm}$ will substantially diffract out of the beam before interacting with the atoms. For the large LMT cases, the averaging that occurs over the laser beam interaction time (which becomes a substantial fraction of a second for large LMT) has also been found to reduce the size of the wavefront aberration noise, by factors of $\sim 100$ for terms that are first order in $\delta$ and of $\sim 10^{4}$ for terms that are second order in $\delta$ for $n \sim 4 \times 10^{4}$ and spatial perturbations of a spatial scale of $\sim 1 \mathrm{~mm}$. To be maximally conservative, this averaging is not included in the phase error estimates here. To reduce wavefront-induced phase noise, it will be important to either control or measure displacements arising from initial kinematic offsets at the level of $1 \mu \mathrm{m}$ or better on each experimental shot. For the anticipated $1 \mathrm{~mm}$ cloud sizes and $10^{6}$ atoms in the initial version of MAGIS-100, measurements at this level are compatible with the atom shot noise limit. For an upgraded atom flux of $10^{8}$ atoms per shot, the atom shot noise limited kinematic resolution is improved to $0.1 \mu \mathrm{m}$ per shot. Moreover, phase shifts from the coupling of initial kinematic jitter to wavefront perturbations can be further reduced using the general kinematic-dependent phase shift compensation technique detailed in Sec. 6.8. Part of the research and development program will be devoted to experimentally implementing this technique for the mitigation of wavefront-induced phase shifts, in combination with developing methods for reducing $\delta$ via a combination of in situ wavefront measurements and deformable optical elements or spatial light modulators. For the ultimate MAGIS-100 interferometer parameters, displacements $\Delta x$ measured and/or controlled at the level of $0.1 \mu \mathrm{m}$ per shot, $\delta=3 \times 10^{-4}$ (improved, e.g., by compensation with deformable optics or spatial light modulators), and a suppression factor of 10 from the compensation technique described in Sec. 6.8, $\delta \phi_{w f, 1} \sim 1 \times 10^{-5} \mathrm{rad} / \sqrt{\mathrm{Hz}}$ and $\delta \phi_{w f, 2} \sim 2 \times 10^{-8} \mathrm{rad} / \sqrt{\mathrm{Hz}}$. Temporally varying wavefront aberrations can also lead to phase noise in the target frequency band. Sources of such variations and their typical sizes are discussed in detail in Ref. [22]. In situ wavefront characterization can be used to measure any such temporal variations and account for their effects in post-processing. In addition to full modeling of the wavefront aberration phase shifts, new schemes for measuring the wavefront with advanced imaging techniques are being developed. Methods involving tilted beam pulse 
sequences can allow for the spatial derivatives of the wavefront to be mapped out across the beam. Further details can be found in Appendix J.

- Seismic vibration. Ground vibration imprints phase noise on the interferometry laser pulse due to vibrations of the delivery optics. This phase noise impacts the detector in the same way as intrinsic laser noise (see above) and also cancels to a high degree as a common mode $[1,23]$. For a velocity mismatch $\Delta v$ between the two clouds in the gradiometer, there is a residual phase shift $\delta \phi_{\text {vibration }} \sim\left(10^{-9} \mathrm{rad} / \sqrt{\mathrm{Hz}}\right)\left(\frac{n}{100}\right)\left(\frac{\Delta v}{10 \mu \mathrm{m} / \mathrm{s}}\right)\left(\frac{T}{1 \mathrm{~s}}\right)\left(\frac{\delta a}{10^{-4} \mathrm{~m} / \mathrm{s}^{2} / \sqrt{\mathrm{Hz}}}\right)$ associated with vibration of the critical beam steering optics with amplitude spectral density $\delta a . T$ is the duration between the interferometer beam splitter and mirror interactions, and all other parameters are as defined above [23]. $\delta \phi_{\text {vibration }}$ is below the fundamental detection noise floor for typical experimental parameters, and $\delta a$ can be reduced as needed by adding modest vibration isolation to critical optical elements. Seismic vibrations are also associated with moving mass in the vicinity of the detector. This can lead to additional background noise due to the gravitational coupling of the moving objects to the atom ensembles (see the discussion of gravity gradient noise in Appendix I).

- Coriolis effects. The rotation of the Earth causes the atom trajectories to deflect from ideal vertical free-fall. For long fall time, the Coriolis force can deflect the atom outside the center of the laser beam, reducing the atom optics efficiency. Variations in the transverse velocity of the cloud cause a substantial Coriolis-induced phase shear across the ensemble that can wash out the interferometer contrast if it is not resolved or reduced. Strategies to compensate the Coriolis force from Earth's rotation are discussed in Section 6. These include counter-rotating the atom optics laser beam against Earth's rotation and implementing multiloop interferometers that are insensitive to rotations (see the bullet point below on 'Initial Cloud Kinematics' for a more detailed discussion). Modeling of Coriolis effects on atom interferometers has been extensively studied and verified in the Stanford $10 \mathrm{~m}$ fountain [60,61], giving confidence that the strategies in Section 6 will work as expected in MAGIS-100.

- Laser pointing jitter. Uncontrolled pointing jitter of the laser causes phase shifts in the interferometer since it changes the position of the atom with respect to the laser wavefronts. The behavior of these phase shifts is well-understood [22,60,61]. Pointing jitter in the target frequency band for the science signal $(\sim 0.1-10 \mathrm{~Hz})$ can act as a noise background. Let $\delta \Phi$ denote the amplitude of the power spectral density for pointing jitter in this band. If the pointing jitter originates from optics near one of the atom ensembles, the laser wavefronts are transversely displaced by a distance $L \delta \Phi$ for the far interferometer and much less for the near interferometer, where $L \approx 100 \mathrm{~m}$ is the baseline length. The dominant source of noise in this situation arises from the coupling of this displacement to laser wavefront aberrations. With the wavefront aberrations parameterized as above, the level of background noise from laser pointing jitter is $\delta \phi_{\text {Pointing }} \sim\left(2 \times 10^{-5} \mathrm{rad} / \sqrt{\mathrm{Hz}}\right)\left(\frac{n}{100}\right)\left(\frac{\delta}{0.005}\right)\left(\frac{\delta \Phi}{1 \mathrm{nrad} / \sqrt{\mathrm{Hz}}}\right)\left(\frac{k_{t}}{(3 \mathrm{~mm})^{-1}}\right)$. Laser pointing will be monitored using a split photodetector (see Ref. [69] for a more detailed discussion), and feedback to control the laser direction can be used if needed. It is noted that angle measurements at the level of $1 \mathrm{prad} / \sqrt{\mathrm{Hz}}$ have been achieved [70]. To reduce $\delta \phi_{\text {Pointing }}$ for the case of increased $n$ and improved phase resolution after research and development, a combination of improved feedback on the pointing angle $\delta \Phi$ and reduction of the beam perturbation amplitude $\delta$ (e.g., employing adaptive optics) can be implemented. For the interferometer parameters after research and development, $\delta=0.001$, and $\delta \Phi=10 \mathrm{prad} / \sqrt{\mathrm{Hz}}$, $\delta \phi_{\text {Pointing }} \sim 1 \times 10^{-5} \mathrm{rad} / \sqrt{\mathrm{Hz}}$. 
- AC Stark shifts. Off-resonant light causes an AC Stark shift of the atomic line. For example, even for resonant excitation of the clock transition in $\operatorname{Sr}(\lambda=698 \mathrm{~nm})$, there is an AC Stark shift due to off-resonant coupling to the other atomic levels. In particular, off-resonant coupling to the ${ }^{1} S_{0} \rightarrow{ }^{1} P_{1}(461 \mathrm{~nm})$ transition shifts the energy of the ground $\left({ }^{1} S_{0}\right)$ state, and offresonant coupling to the ${ }^{3} P_{0} \rightarrow{ }^{3} S_{1}(679 \mathrm{~nm})$ transition shifts the energy of the excited clock $\left({ }^{3} P_{0}\right)$ state [76]. This energy shift can cause a phase shift in an interferometer that mimics the target signal to the extent that it varies spatially and fluctuates in time in the target frequency band. Intensity fluctuations of the laser are a dominant source of this, so laser intensity control can reduce the effect. For interferometers with $n \hbar k$ beam splitters, phase backgrounds from AC Stark shifts are at the level of $\delta \phi_{\mathrm{AC}} \sim\left(6 \times 10^{-5} \mathrm{rad} / \sqrt{\mathrm{Hz}}\right)\left(\frac{n}{100}\right)\left(\frac{\delta I / I}{10^{-6} / \sqrt{\mathrm{Hz}}}\right)$, where $\delta I / I$ is the amplitude of the power spectral density in the target frequency band for the fractional fluctuation of the differential laser intensity (averaged over the atom ensembles) between the two interferometers. $\delta I / I \sim 10^{-6} / \sqrt{\mathrm{Hz}}$ can be realized, for example, with a $0.1 \%$ spatial intensity variation between the two interferometers and laser intensity stabilization at the level of $0.1 \%$, which is readily achievable. Moreover, it is possible to engineer the spectrum of the laser to suppress AC Stark shifts, making use of light detuned both above and below a transition [17]. This AC Stark shift compensation can further relax requirements on intensity stability. For reference, we note that in [77], intensity fluctuations were reduced to the level of $6 \times 10^{-7} f^{-1 / 2} / \sqrt{\mathrm{Hz}}$ for frequencies $f$ down to $\sim 0.1 \mathrm{~Hz}$. For the ultimate interferometer parameters after research and development and for $0.1 \%$ spatial intensity variation, this level of intensity control corresponds to $\delta \phi_{\mathrm{AC}} \sim 1 \times 10^{-5} \mathrm{rad} / \sqrt{\mathrm{Hz}}$ (not assuming any additional suppression from AC Stark compensation). Transverse-position-dependent AC Stark shifts can also couple to initial atom kinematic jitter in a manner analogous to wavefront aberrations (discussed above). For a given intensity/wavefront perturbation amplitude, phase errors from AC Stark couplings of this type will be slightly smaller than the corresponding wavefront-induced phase errors. For such AC Stark couplings, analogous mitigation strategies can be applied as in the wavefront case. In situ measurements of intensity perturbations can be measured by implementing spatially resolved detection combined with short duration interferometers in a superposition of different internal states and leaving on a long Dopplerdetuned laser pulse.

- Initial cloud kinematics. Fluctuations in the initial position and velocity of the atom ensembles can cause unwanted phase shifts in a number of ways. Shot-to-shot fluctuations in the atom trajectory can couple to rotations, wavefront aberrations, and spatial variations in external fields (e.g., magnetic or gravity gradients) and lead to time dependent phases errors. Such effects have been theoretically analyzed [21,22, 58], and they have also been experimentally investigated in the Stanford $10 \mathrm{~m}$ fountain $[12,60,61]$. Mitigation strategies for initial cloud kinematic jitter are discussed in Section 6. From the discussion of wavefront perturbations above, it is important to control or measure trajectory variations arising from initial kinematic jitter at the $1 \mu \mathrm{m}$ level on each experimental shot. Multi-loop interferometers provide a way to cancel phase shifts from the coupling of initial kinematics to gravity gradients or rotations while preserving the time-varying dark matter or gravitational wave signal $[22,68]$. As an illustrative example, a three-loop interferometer configured to cancel out leading order phase shift contributions from rotations and gravity gradients is considered [68]. In such an interferometer, the coupling of higher-order rotation/gravity gradient phase shifts to initial kinematic jitter can still be a source of noise. The dominant such noise term arises from a cross-coupling between rotations, gravity gradients, and initial atom ve- 
locity and has the form $\delta \phi_{\mathrm{RGGV}}=(17 / 3+4 \sqrt{2}) n k \Delta v_{x} \Omega_{y} T_{z z} T^{4}$. Here, the $x, y$, and $z$ axes are defined so that $z$ is normal to Earth's surface and Earth's rotation vector lies in the $y z$ plane. $\Delta v_{x}$ denotes the shot-to-shot jitter in the atom cloud velocity along the $x$ axis (or the accuracy to which this jitter can be measured on each experimental shot if post-processing corrections are implemented), $\Omega_{y}$ is the $y$ component of Earth's rotation vector, $T_{z z}$ is the vertical gravity gradient, $T$ is the duration between the first beam splitter and mirror interactions, and all other notation is as defined above. The associated noise has the magnitude $\delta \phi_{\mathrm{RGGV}} \sim\left(2 \times 10^{-6} \mathrm{rad} / \sqrt{\mathrm{Hz}}\right)\left(\frac{n}{100}\right)\left(\frac{\Delta v_{x}}{1 \mu \mathrm{m} / \mathrm{s} / \sqrt{\mathrm{Hz}}}\right)\left(\frac{T}{1 \mathrm{~s}}\right)$. For the ultimate interferometer parameters after research and development, and assuming measurement of $\Delta v_{x}$ at the level of $0.1 \mu \mathrm{m}$ on each experimental shot (this corresponds to the shot noise limit for $10^{8}$ atoms and a $1 \mathrm{~mm}$ cloud size), $\delta \phi_{\mathrm{RGGV}} \sim 6 \times 10^{-5} \mathrm{rad} / \sqrt{\mathrm{Hz}}$. Additional loops can be added as needed to further suppress phase shifts from the cross-coupling of rotations, gravity gradients, and initial atom velocity. Moreover, as discussed in Sec. 6.8, the residual coupling of initial kinematics to rotations and gravity gradients can be further suppressed by appropriate counteracting phase shifts arising from mirror tilts. Assuming a modest factor of 10 reduction in $\delta \phi_{\mathrm{RGGV}}$ from this compensation technique, $\delta \phi_{\mathrm{RGGV}} \sim 6 \times 10^{-6} \mathrm{rad} / \sqrt{\mathrm{Hz}}$ for the ultimate interferometer parameters. An alternative method to compensate phase shifts from gravity gradients by slightly adjusting the momentum transferred during the interferometer mirror sequence has recently been theoretically proposed [78] and experimentally demonstrated [12,79]. In MAGIS-100, this momentum transfer adjustment could be achieved by incorporating a small number of Bragg transitions into the interferometer using light with a different, finely tunable wavelength.

- Mean field shifts. Atom-atom interactions cause an energy shift of the clock transition proportional to the atomic density. This can cause a systematic error in an interferometer if the two arms occupy different atomic states (with different mean field shifts), or if the density is asymmetric. The detector is sensitive to any time-varying mean field shifts, which may arise if the density fluctuates shot-to-shot. This background can be suppressed by using ensembles with low density (after matter wave lensing), by employing sequences that use symmetric internal states for the two arms, and by using symmetric beam splitting sequences $[12,80]$. For ${ }^{87} \mathrm{Sr}$, the mean field shift has been measured to be $\sim(0.1 \mathrm{~Hz})\left(\frac{n_{\text {atom }}}{10^{11} \text { atoms } / \mathrm{cm}^{3}}\right)$ [71], where $n_{\text {atom }}$ is the atom number density. The mean field shift arises when atoms are inhomogeneously excited on the clock transition, as otherwise atom-atom interactions are suppressed by Pauli blocking [71]. Therefore, the times at which mean field shifts will affect phase evolution in MAGIS-100 are during the atom optics pulses. The initial version of MAGIS-100 will have $\sim 10^{6}$ atoms per interferometer in a volume of $\sim 1 \mathrm{~mm}^{3}$. Using these numbers and assuming $n=100$ and a $\pi$-pulse duration of $200 \mu \mathrm{s}$, phase backgrounds from mean field shifts are $\delta \phi_{\mathrm{MF}} \sim\left(5 \times 10^{-4} \mathrm{rad} / \sqrt{\mathrm{Hz}}\right)\left(\delta N_{A} / N_{A}\right)$, where $\delta N_{A} / N_{A}$ is the shot-to-shot fluctuation of the fractional atom population asymmetry between the two interferometer arms (the relevant fluctuations are those in the target frequency band). Controlling this asymmetry at the $0.1 \%$ level, which is not difficult to achieve with laser intensity stabilization, will reduce mean field backgrounds well below $10^{-3} \mathrm{rad} / \sqrt{\mathrm{Hz}}$. Additionally, closely matching the densities of the two separated interferometers will allow the mean field phase shift to be further suppressed as a common mode, and highly robust beam splitters using composite pulses [81] can dramatically reduce fluctuations in the population asymmetry. For the interferometer parameters corresponding to the ultimate sensitivity goal after research and development, $\delta \phi_{\mathrm{MF}}$ can be kept at the level of $2 \times 10^{-6} \mathrm{rad} / \sqrt{\mathrm{Hz}}$ by stabilizing (or measuring) the relative densities 
between the two separated interferometers to $1 \%$ and employing composite pulses to reduce $\delta N_{A} / N_{A}$ to $10^{-5}$.

- Magnetic fields. The clock energy levels of Sr shift in response to magnetic fields. Time varying magnetic fields can cause systematic frequency shifts that behave like a gravitational wave or ultralight dark matter signal. As discussed in Section 6, magnetic shielding will be employed to reduce the influence of stray fields in the interferometer region. Using multiple sequential transitions, the atom interferometry sequence can be designed so that both arms of the interferometer spend most of the time in the ground state, reducing the differential phase shifts. For ${ }^{87} \mathrm{Sr}$, a co-magnetometer can be realized by simultaneously operating two interferometers using states with opposite magnetic field response, suppressing the linear response to magnetic fields and allowing the magnetic field dependent phase shift to be measured and subtracted. The residual quadratic Zeeman shift coefficient is $-0.23 \mathrm{~Hz} / \mathrm{G}^{2}$ [64], implying that control of magnetic field variations at the level of $\sim 1 \mathrm{mG} / \sqrt{\mathrm{Hz}}$ in the relevant band should be more than sufficient for the target sensitivity goal. Magnetic shielding to the $1 \mathrm{mG}$ level is implemented in the Stanford $10 \mathrm{~m}$ fountain [63]. In MAGIS-100 the field will be monitored with magnetometers and shielding to the $10 \mathrm{mG}$ level will be adequate to achieve the sensitivity goal for signals in the frequency range of interest.

- Blackbody radiation shifts. Blackbody radiation causes an energy shift of the atomic energy levels. This can result in phase noise in the interferometer if the temperature of the vacuum tube varies in time in the target frequency band. For the strontium clock transition, the blackbody shift has a temperature coefficient of $-2.3 \mathrm{~Hz}\left(\frac{T_{\text {system }}}{300 \mathrm{~K}}\right)^{4}$, where $T_{\text {system }}$ is the temperature of the apparatus [64]. It is important to note that apparatus temperature drifts will naturally occur at frequencies much lower than the target frequency band. For a three-loop interferometer with $T$ as defined above and a temperature oscillation at frequency $\omega_{\text {Temp }}$, the interferometer phase response to temperature variations at low frequency $\omega_{\text {Temp }}$ is suppressed by a factor of $\left(\omega_{\mathrm{Temp}} T\right)^{2}$. For a temperature oscillation of amplitude $1 \mathrm{~K}$ and period 1 hour, the associated interferometer noise is at the level of $\sim 1 \times 10^{-6} \mathrm{rad} / \sqrt{\mathrm{Hz}}$.

- Imaging aberrations. Aberrations in the lens system used to image the atom fringe pattern can introduce apparent phase shifts. Assuming the aberrations are not uniform over the field of view of the imaging system, a time-varying effect may arise if the position of the interference pattern changes shot-to-shot due to initial atom kinematics. This effect has been characterized in the Stanford $10 \mathrm{~m}$ fountain both by making use of ray-tracing software to analyze the imaging lens system and by controllably measuring phase shifts as a function of position on the camera. The same methods can be applied to MAGIS-100. It is anticipated that such characterization, in combination with atom cloud kinematics controlled and/or measured at the $1 \mu \mathrm{m}$ level on each shot, will make phase errors from imaging aberrations negligible.

- Timing jitter. A timing-jitter induced asymmetry $\delta T$ in the duration of different free propagation zones of the interferometer, in combination with a velocity mismatch $\Delta v$ between the two clouds in the gradiometer, leads to interferometer phase noise of magnitude $\delta \phi_{\text {timing }} \sim$ $\left(10^{-8} \mathrm{rad} / \sqrt{\mathrm{Hz}}\right)\left(\frac{n}{100}\right)\left(\frac{\Delta v}{10 \mu \mathrm{m} / \mathrm{s}}\right)\left(\frac{\delta T}{1 \mathrm{ps} / \sqrt{\mathrm{Hz}}}\right)$. Timing jitter of $1 \mathrm{ps} / \sqrt{\mathrm{Hz}}$ or lower in the relevant band can be readily by achieved by referencing all critical frequencies to a master oscillator with good short-term stability. 
- Background gas index of refraction. The index of refraction from background gas in the pipe modifies the optical path length associated with the baseline. Noise $\delta \eta$ in the index of refraction $\eta$ therefore leads to a spurious strain signal $\delta h_{\text {index }}=\delta \eta$ [21]. The index of refraction of air is $\eta \sim 1+3 \times 10^{-4}\left(\frac{P}{760 \text { Torr }}\right)\left(\frac{300 \mathrm{~K}}{T_{\text {system }}}\right)$, where $P$ is the pressure and $T_{\text {system }}$ is the temperature of the system. The spurious strain signal associated with index of refraction variation due to temperature fluctuation $\delta T_{\text {system }}$ with a period of 1 hour (for $T=1 \mathrm{~s}$ ) is $\delta h_{\text {index }}=$ $\delta \eta \sim\left(4 \times 10^{-25} / \sqrt{\mathrm{Hz}}\right)\left(\frac{P}{10^{-10} \text { Torr }}\right)\left(\frac{300 \mathrm{~K}}{T_{\text {system }}}\right)\left(\frac{\delta T_{\text {system }}}{1 \mathrm{~K}}\right)$. The spurious strain signal associated with index of refraction variation due to fractional pressure fluctuation $\delta P / P$ in the frequency band of interest is $\delta h_{\text {index }}=\delta \eta \sim\left(4 \times 10^{-20} / \sqrt{\mathrm{Hz}}\right)\left(\frac{P}{10^{-10} \text { Torr }}\right)\left(\frac{300 \mathrm{~K}}{T_{\text {system }}}\right)\left(\frac{\delta P / P}{0.001 / \sqrt{\mathrm{Hz}}}\right)$. For reference, it is noted that the same index of refraction effects apply to LIGO, which maintains ultra-high vacuum of $10^{-8}$ to $10^{-9}$ Torr [82]. Additionally, the index of refraction of the ultracold atom clouds will induce a phase shift on the laser beam when it passes through a cloud. The dominant effect on the index of refraction will come from the strong $461 \mathrm{~nm}$ and $679 \mathrm{~nm}$ transitions. Interferometer phase noise from this effect has the magnitude $\delta \phi_{\text {cloud }} \sim\left(10^{-8} \mathrm{rad} / \sqrt{\mathrm{Hz}}\right)\left(\frac{n}{100}\right)\left(\frac{N_{\text {Atom }}}{10^{6}}\right)\left(\frac{r_{\text {cloud }}}{1 \mathrm{~mm}}\right)^{2}\left[\left(\frac{\delta N_{\text {Atom }} / N_{\text {Atom }}}{0.01 / \sqrt{\mathrm{Hz}}}\right)^{2}+4\left(\frac{\delta r_{\text {cloud }} / r_{\text {cloud }}}{0.01 / \sqrt{\mathrm{Hz}}}\right)^{2}\right]^{1 / 2}$, where $N_{\text {Atom }}$ is the atom number in a given experimental shot, $r_{\text {cloud }}$ is the atom cloud radius, $\delta N_{\text {Atom }} / N_{\text {Atom }}$ is the fractional shot-to-shot fluctuation in the atom number, $\delta r_{\text {cloud }} / r_{\text {cloud }}$ is the fractional shot-to-shot fluctuation in the cloud radius, and (as before) $n$ is the number of beam splitter momentum kicks. If needed, $\delta N_{\text {Atom }} / N_{\text {Atom }}$ and $\delta r_{\text {cloud }} / r_{\text {cloud }}$ can be measured on each shot and associated phase shifts can be subtracted in post-processing. For the goal parameters after research and development, phase noise from index of refraction effects can be kept to $3 \times 10^{-6} \mathrm{rad} \sqrt{\mathrm{Hz}}$ by measuring the atom number and cloud radius at the fractional level of $10^{-4}$ on each shot.

Total uncertainties are summarized in Table 11.

\begin{tabular}{|c|c|c|}
\hline Source of Noise & Size for Initial Configuration & Size for 5 year Configuration \\
\hline Wavefront & $5 \times 10^{-5} \mathrm{rad} / \sqrt{\mathrm{Hz}}$ & $1 \times 10^{-5} \mathrm{rad} / \sqrt{\mathrm{Hz}}$ \\
Pointing Jitter & $2 \times 10^{-5} \mathrm{rad} / \sqrt{\mathrm{Hz}}$ & $1 \times 10^{-5} \mathrm{rad} / \sqrt{\mathrm{Hz}}$ \\
AC Stark Shifts & $6 \times 10^{-5} \mathrm{rad} / \sqrt{\mathrm{Hz}}$ & $1 \times 10^{-5} \mathrm{rad} / \sqrt{\mathrm{Hz}}$ \\
Initial Cloud Kinematics & $2 \times 10^{-6} \mathrm{rad} / \sqrt{\mathrm{Hz}}$ & $6 \times 10^{-6} \mathrm{rad} / \sqrt{\mathrm{Hz}}$ \\
Other & $5 \times 10^{-6} \mathrm{rad} / \sqrt{\mathrm{Hz}}$ & $5 \times 10^{-6} \mathrm{rad} / \sqrt{\mathrm{Hz}}$ \\
\hline Total Uncertainty & $\mathbf{6} \times \mathbf{1 0}^{-\mathbf{4}} \mathbf{r a d} / \sqrt{\mathbf{H z}}$ & $\mathbf{2} \times \mathbf{1 0}^{-\mathbf{5}} \mathrm{rad} / \sqrt{\mathbf{H z}}$ \\
\hline
\end{tabular}

Table 11: Summary of contributing noise backgrounds for the initial MAGIS-100 configuration and for the configuration after 5 years of research and development. The dominant noise backgrounds discussed above are listed individually, while the remaining contributions are combined under the category 'Other.' It is assumed here that the noise sources can validly be added in quadrature. The assumptions that lead to the numbers shown here are detailed in the bulleted list above. 


\section{Appendix: Gravity Gradient Noise}

Because the atoms in MAGIS-100 are free-falling and the interferometers are run using single and common laser pulses, seismic activity does not directly lead to noise in the measurement. However, the atomic clouds are coupled to the shaking ground through Newtonian gravity. This effect, known as gravity gradient noise (GGN), is a sub-dominant source of noise at LIGO at most frequencies and has been studied in detail [83]. Particularly near the surface, GGN is often dominated by Rayleigh waves, which are seismic surface waves which travel at a speed of $c_{R} \sim 50-300 \mathrm{~m} / \mathrm{s}$. The GGN noise can be estimated in two regimes - when the wavelength of the Rayleigh waves $\lambda_{R}=c_{R} / f$ are either longer or shorter than the experimental baseline $L$. An estimate for the GGN strain noise in MAGIS-100 is

$$
h_{\mathrm{GGN}} \sim \begin{cases}\frac{G_{N} \rho}{c_{R} f}\left\langle\delta x_{\text {seismic }}\right\rangle & f<\frac{c_{R}}{2 \pi L} \\ \frac{G_{N} \rho}{2 \pi f^{2}} \frac{\left\langle\delta x_{\text {seismic }}\right\rangle}{L} & f>\frac{c_{R}}{2 \pi L}\end{cases}
$$

where $\rho$ is the mass density of the surrounding rock, and $\left\langle\delta x_{\text {seismic }}\right\rangle$ is the average seismic displacement per $\sqrt{\mathrm{Hz}}$. Assuming $\left\langle\delta x_{\text {seismic }}\right\rangle \sim 1 \mu \mathrm{m}$ we estimate $h_{\mathrm{GGN}} \sim 10^{-16}$ at $1 \mathrm{~Hz}$. A characterization of the seismic activity near the NuMI shaft is underway and will further inform this estimate.

MAGIS-100 will attempt to measure and characterize the GGN in the lower frequency regime. This will be useful for mitigation purposes as well as being of interest to the geophysics community. An atomic interferometer at this scale is a sensitive gradiometer that can be used for understanding local seismic activity, gravitational field perturbations caused by density shifts, i.e. slip faults, and other geological irregularities as discussed by Ref. [24]. 


\section{J Appendix: Laser Wavefront Aberration}

\section{J.1 Calculation}

The following discusses the interferometer phase response to a laser beam perturbation. Consider a Fourier component with amplitude $\delta$ and transverse spatial frequency $k_{x}$. The beam is treated paraxially, which means that the wavevector, $\vec{k}$, is nearly parallel to the longitudinal axis down the pipe. The coordinate system is defined so that the $x y$-plane is transverse to the laser propagation and the $z$-axis is in the direction of propagation, which is vertical. The $x$ and $y$ axes are defined so that Earth's rotation vector lies in the $y z$ plane. As an illustrative example, a perturbation Fourier component $k_{x}$ along the $x$-axis is considered. An analogous treatment applies for a Fourier component along a general axis in the $x y$-plane. For the phase calculation, the equations of motion of the atoms are solved by expanding in a power series and keeping terms up to the third order in time. Also, only Earth rotations $\Omega_{y}$ are considered, as these couple with the $x$ and $z$ positions of the atoms and the vertical launch velocity. By contrast, terms including $\Omega_{z}$ couple with the $y$ position of the atom and the transverse launch velocities, which are much smaller. This allows the problem to be reduced to a two dimensional phase calculation in the $x z$-plane. The final terms ignored are the higher order gravity gradients that arise from a Taylor expansion of the gravitational potential field.

The laser beam field takes the general form

$$
E(x, z)=u(x, z) e^{i k z} .
$$

For an initial perturbation Fourier component $\delta \cos \left(k_{x} x\right)$ in the laser field, the initial field and the field at some farther distance are given by multiplying the perturbation by the corresponding paraxial propagator:

$$
\begin{aligned}
& u(x, 0)=1+\delta \cos \left(k_{x} x\right), \\
& u(x, z)=1+\delta \cos \left(k_{x} x\right) e^{i \frac{k_{x}^{2}}{2 k} z}
\end{aligned}
$$

for a beam propagating in the $-z$ direction [84]. The paraxial propagator follows from considering the $z$-component, $k_{z}$, of the wave vector corresponding to transverse Fourier component $k_{x}$. Since the total wave vector of this Fourier component must have amplitude $k$, we require that $k=$ $\sqrt{k_{z}^{2}+k_{x}^{2}}$. Therefore,

$$
k_{z}=\sqrt{k^{2}-k_{x}^{2}} \approx k-\frac{k_{x}^{2}}{2 k}
$$

The difference between $k_{z}$ and $k$ leads to the paraxial propagator $e^{-i\left(k_{z}-k\right) z}=e^{i \frac{k_{x}^{2}}{2 k} z}$. Eq. (5b) corresponds to amplitude and phase perturbations

$$
\begin{aligned}
\text { Amplitude: } & \delta \cos \left(k_{x} x\right) \cos \left(\frac{k_{x}^{2}}{2 k} z\right) \\
\text { Phase: } & \phi_{w}=\delta \cos \left(k_{x} x\right) \sin \left(\frac{k_{x}^{2}}{2 k} z\right) .
\end{aligned}
$$

Local spatial gradients of Equation (7b) determine additional contributions to the local wave vector 


$$
\begin{gathered}
\delta k_{x}=\frac{\partial \phi_{w}}{\partial x}=-k_{x} \delta \sin \left(k_{x} x\right) \sin \left(\frac{k_{x}^{2}}{2 k} z\right) \\
\delta k_{z}=\frac{\partial \phi_{w}}{\partial z}=\frac{k_{x}^{2}}{2 k} \delta \cos \left(k_{x} x\right) \cos \left(\frac{k_{x}^{2}}{2 k} z\right)
\end{gathered}
$$

A laser pulse delivers a momentum kick to an atom equal to $\hbar \vec{k}$, where $\vec{k}$ is the local wave vector at the atom's location [85]. The perturbations $\delta k_{x}$ and $\delta k_{z}$ therefore cause the atoms to receive additional momentum kicks proportional to the perturbation size $\delta$.

First, the effect of the beam perturbation on the laser phase that is imprinted on the atoms as $\phi_{\text {laser }}=n\left(k z+\phi_{w}\right)$ is considered, where $n$ is the size of the large momentum transfer to the atom, at each laser-atom interaction point. The effect on the propagation and separation phases will be considered subsequently.

The total laser phase built up after a three-pulse sequence $(\pi / 2, \pi, \pi / 2)$ with laser-atom interactions occurring at spacetime points $A, B, C$, and $D$ [58] (see Fig. 5) is defined as

$$
\Delta \phi_{\text {laser }}=\phi_{A}-\phi_{B}-\phi_{C}+\phi_{D}
$$

where $\phi_{i}$ is the laser phase evaluated at spacetime point $i$. For simplicity, momentum kicks resulting from $\delta k_{x}$ and $\delta k_{z}$ (which are referred to from here on as the $\delta k$ kicks) are ignored for the moment. Rotations are also ignored for the moment. The laser phases imprinted at the four interaction points are

$$
\begin{aligned}
\phi_{A}=n & \left(\delta \cos \left(k_{x} x_{i}\right) \sin \left(\frac{k_{x}^{2} z_{i}}{2 k}\right)+k z_{i}\right) \\
\phi_{B}=n\left(\delta \cos \left(k_{x}\left(T v_{x}+x_{i}\right)\right) \sin \left(\frac{k_{x}^{2}\left(-\frac{g T^{2}}{2}+T\left(\frac{k n \hbar}{m}+v_{z}\right)+z_{i}\right)}{2 k}\right)\right. & \\
\left.+k\left(-\frac{g T^{2}}{2}+T\left(\frac{k n \hbar}{m}+v_{z}\right)+z_{i}\right)\right) & \left(\delta \cos \left(k_{x}\left(T v_{x}+x_{i}\right)\right) \sin \left(\frac{k_{x}^{2}\left(-\frac{g T^{2}}{2}+T v_{z}+z_{i}\right)}{2 k}\right)+k\left(-\frac{g T^{2}}{2}+T v_{z}+z_{i}\right)\right) \\
\phi_{C}=n & \left(\delta \cos \left(k_{x}\left(2 T v_{x}+x_{i}\right)\right) \sin \left(\frac{k_{x}^{2}\left(-2 g m T^{2}+k n T \hbar+2 m T v_{z}+m z_{i}\right)}{2 k m}\right)\right. \\
\phi_{D}=n & \left.+k\left(T\left(-2 g T+\frac{k n \hbar}{m}+2 v_{z}\right)+z_{i}\right)\right) .
\end{aligned}
$$


Combining these contributions per Eq. (9), the total laser phase shift reduces to

$$
\begin{aligned}
\Delta \phi_{\text {laser }} & =-g k n T^{2}+\left[-n \cos \left(2 k_{x} T v_{x}+k_{x} x_{i}\right) \sin \left(\frac{g k_{x}^{2} T^{2}}{k}-\frac{k_{x}^{2} T v_{z}}{k}-\frac{k_{x}^{2} z_{i}}{2 k}-\frac{k_{x}^{2} n T \hbar}{2 m}\right)\right. \\
& +n \cos \left(k_{x} T v_{x}+k_{x} x_{i}\right) \sin \left(\frac{g k_{x}^{2} T^{2}}{4 k}-\frac{k_{x}^{2} T v_{z}}{2 k}-\frac{k_{x}^{2} z_{i}}{2 k}-\frac{k_{x}^{2} n T \hbar}{2 m}\right) \\
& +n \cos \left(k_{x} T v_{x}+k_{x} x_{i}\right) \sin \left(\frac{g k_{x}^{2} T^{2}}{4 k}-\frac{k_{x}^{2} T v_{z}}{2 k}-\frac{k_{x}^{2} z_{i}}{2 k}\right) \\
& \left.+n \cos \left(k_{x} x_{i}\right) \sin \left(\frac{k_{x}^{2} z_{i}}{2 k}\right)\right] \delta .
\end{aligned}
$$

Without including the $\delta k$ kicks, the entire phase contribution from wavefront perturbations enters through the laser phase [22]. When a full phase shift calculation including these momentum kicks is performed-which requires including contributions from the laser, propagation, and separation phases to find the total phase difference $\Delta \phi_{\text {total }}=\Delta \phi_{\text {prop }}+\Delta \phi_{\text {laser }}+\Delta \phi_{\text {sep }}[58]$-the same answer is obtained to leading order in $\delta$. In the full calculation, new phase contributions are obtained at second order in $\delta$. In order to keep the terms to a manageable number, physical cuts are made based on the size of product terms. Large numbers were multiplied by a factor $1 / \varepsilon$ and small numbers by a factor $\varepsilon$. In the final result, $\varepsilon$ was set to zero to cut terms that were negligible. The total phase difference was then expanded to second order in $\delta$. A list of the zeroth, first, and second order terms can be found in Tables 12-15. This calculation differs from the previous calculation [22], because a full Fourier analysis including transverse and longitudinal kicks caused by the wavefront aberration has been performed. The second order terms can be thought of as arising from the recoil kinetic energy induced by the momentum kicks $\delta k_{x}$ and $\delta k_{z}$. These terms are expected physically, and some of the terms are listed below.

\section{J.2 Leading Order Terms}

Here are listed some of the leading terms for two versions of the calculation. The first, simpler set does not include the Earth's rotation, as this adds many coupled terms to the phase shift, and the second set includes Earth's rotation. Terms that are coupled to Earth's rotation can be suppressed by the same rotation compensation system used to suppress terms arising from the Coriolis effect.

\begin{tabular}{|c|c|}
\hline Term & Phase shift \\
\hline \hline 1 & $n \cos \left(k_{x} x_{i}\right) \sin \left(\frac{k_{x}^{2} z_{i}}{2 k}\right)$ \\
2 & $n \cos \left(k_{x} T v_{x}+k_{x} x_{i}\right) \sin \left(\frac{g k_{x}^{2} T^{2}}{4 k}-\frac{k_{x}^{2} T v_{z}}{2 k}-\frac{k_{x}^{2} z_{i}}{2 k}\right)$ \\
3 & $-n \cos \left(2 k_{x} T v_{x}+k_{x} x_{i}\right) \sin \left(\frac{g k_{x}^{2} T^{2}}{k}-\frac{k_{x}^{2} T v_{z}}{k}-\frac{k_{x}^{2} z_{i}}{2 k}-\frac{k_{x}^{2} n T \hbar}{2 m}\right)$ \\
4 & $n \cos \left(k_{x} T v_{x}+k_{x} x_{i}\right) \sin \left(\frac{g k_{x}^{2} T^{2}}{4 k}-\frac{k_{x}^{2} T v_{z}}{2 k}-\frac{k_{x}^{2} z_{i}}{2 k}-\frac{k_{x}^{2} n T \hbar}{2 m}\right)$ \\
\hline
\end{tabular}

Table 12: First order in $\delta$ phase shift terms without Earth rotation. Listed are the coefficients of $\delta$ for each term.

\section{J.3 Wavefront Imaging}

To mitigate wavefront-perturbation-induced phase shifts, it will be important to characterize the laser wavefront in situ using the MAGIS-100 atomic interferometer. Two methods have been 


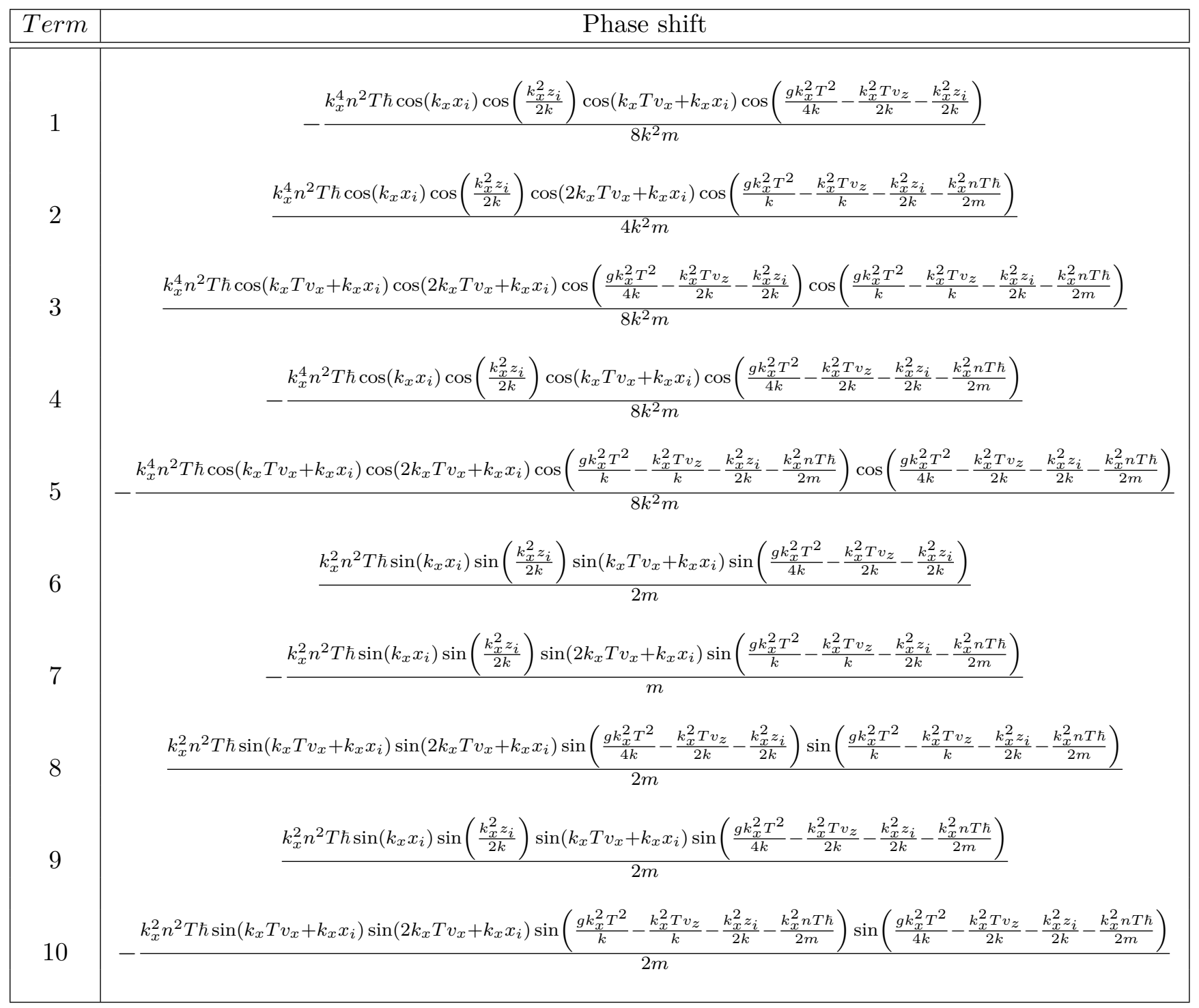

Table 13: Second order in $\delta$ phase shift terms without Earth rotation. Listed are the coefficients of $\delta^{2}$ for each term.

thought of so far and can be used in conjunction with one another. First, a three-pulse laser sequence can be implemented where for the third and final beam splitting pulse one of the tiptilt mirrors is tilted slightly to add an angle $\theta$ to laser beam. This shifts the wavefront by a distance $d_{\text {atom }} \theta$ before the final pulse ( $d_{\text {atom }}$ is the distance between the mirror and the location of the atoms), allowing a measurement of the first derivative of the wavefront across the entire atom cloud to be made using spatially resolved detection and a bottom view of the cloud (see Sec. 6.10). Having both the down-shooting and retro-reflection mirrors mounted on piezo tip-tilt stages will allow wavefront perturbations on the upward and downward propagating beams to be independently characterized. Another method is to purposefully apply an initial transverse velocity kick to the atom cloud, which would provide information about spatial variation of the wavefront along the kick direction. For the laser tilting method, care must be used with the range of angles that can be applied. If too much tilt is added, the frequencies of the atom fringes can grow so large 


\begin{tabular}{|c|c|}
\hline Term & Phase shift \\
\hline \hline 1 & $-g k n T^{2}$ \\
2 & $2 k n T^{2} v_{x} \Omega_{y}$ \\
3 & $k n R T^{2} \Omega_{y}^{2}$ \\
4 & $-\frac{3 k^{2} n^{2} T^{3} \Omega_{y}^{2} \hbar}{2 m}$ \\
\hline
\end{tabular}

Table 14: Zeroth order in $\delta$ phase shift terms with Earth rotation. Listed are the coefficients of $\delta$ for each term. Terms coupling to Earth's rotation, $\Omega_{y}$, can be suppressed via rotation compensation.

\begin{tabular}{|c|c|}
\hline Term & Phase shift \\
\hline \hline 1 & $-\frac{3 k_{x}^{2} n^{2} T^{3} \Omega_{y}^{2} \hbar \cos \left(k_{x} x_{i}\right) \cos \left(\frac{k_{x}^{2} z_{i}}{2 k}\right)}{4 m}$ \\
2 & $\frac{k k_{x} n^{2} T^{2} \Omega_{y} \hbar \sin \left(\frac{1}{3} g k_{x} T^{3} \Omega_{y}-\frac{1}{3} k_{x} R T^{3} \Omega_{y}^{3}-k_{x} T^{2} v_{z} \Omega_{y}+k_{x} T v_{x}+k_{x} x_{i}\right) \sin \left(\frac{g k_{x}^{2} T^{2}}{4 k}-\frac{k_{x}^{2} R T^{2} \Omega_{y}^{2}}{4 k}-\frac{k_{x}^{2} T^{2} v_{x} \Omega_{y}}{2 k}-\frac{k_{x}^{2} T v_{z}}{2 k}-\frac{k_{x}^{2} z_{i}}{2 k}\right)}{m}$ \\
\hline
\end{tabular}

Table 15: Examples of first order in $\delta$ phase shift terms with Earth rotation. Listed are the coefficients of $\delta$ for each term. Terms coupling to Earth's rotation, $\Omega_{y}$, can be suppressed via rotation compensation.

as to be unresolvable by the imaging system. 